\title{
EXCHANGE RATES AND INSULATION IN EMERGING MARKETS
}

Barry Eichengreen, Donghyun Park, Arief Ramayandi, and Kwanho Shin

NO. 610

February 2020
ADB ECONOMICS WORKING PAPER SERIES 


\section{ADB Economics Working Paper Series}

\section{Exchange Rates and Insulation in Emerging Markets}

Barry Eichengreen, Donghyun Park, Arief Ramayandi, and Kwanho Shin

No. 610 | February 2020
Barry Eichengreen (eichengr@berkeley.edu) is a professor at the Department of Economics, University of California Berkeley. Donghyun Park (dpark@adb.org) and Arief Ramayandi (aramayandi@adb.org) are principal economists at the Economic Research and Regional Cooperation Department, Asian Development Bank. Kwanho Shin (khshin@korea.ac.kr) is a professor at the Department of Economics, Korea University.

This paper was prepared as background material for the Asian Development Outlook 2018 Update on "Maintaining Stability amid Heightened Uncertainty." For comments we thank Ila Patnaik and other workshop participants in the Asian Development Outlook Update Midterm Workshop. We also thank Dohoon Kim for excellent research assistance; and Mahvash S. Qureshi, Beth Anne Wilson, and Andrea Raffo for help with the data. 
(C) 2020 Asian Development Bank 6 ADB Avenue, Mandaluyong City, 1550 Metro Manila, Philippines

Tel +632 8632 4444; Fax +63286362444

www.adb.org

Some rights reserved. Published in 2020.

ISSN 2313-6537 (print), 2313-6545 (electronic)

Publication Stock No. WPS200078-2

DOI: http://dx.doi.org/10.22617/WPS200078-2

The views expressed in this publication are those of the authors and do not necessarily reflect the views and policies of the Asian Development Bank (ADB) or its Board of Governors or the governments they represent.

ADB does not guarantee the accuracy of the data included in this publication and accepts no responsibility for any consequence of their use. The mention of specific companies or products of manufacturers does not imply that they are endorsed or recommended by ADB in preference to others of a similar nature that are not mentioned.

By making any designation of or reference to a particular territory or geographic area, or by using the term "country" in this document, $A D B$ does not intend to make any judgments as to the legal or other status of any territory or area.

This work is available under the Creative Commons Attribution 3.0 IGO license (CC BY 3.0 IGO)

https://creativecommons.org/licenses/by/3.0/igo/. By using the content of this publication, you agree to be bound by the terms of this license. For attribution, translations, adaptations, and permissions, please read the provisions and terms of use at https://www.adb.org/terms-use\#openaccess.

This CC license does not apply to non-ADB copyright materials in this publication. If the material is attributed to another source, please contact the copyright owner or publisher of that source for permission to reproduce it. $\mathrm{ADB}$ cannot be held liable for any claims that arise as a result of your use of the material.

Please contact pubsmarketing@adb.org if you have questions or comments with respect to content, or if you wish to obtain copyright permission for your intended use that does not fall within these terms, or for permission to use the ADB logo.

Corrigenda to ADB publications may be found at http://www.adb.org/publications/corrigenda.

Notes:

In this publication, "\$” refers to United States dollars.

ADB recognizes "Korea" as the Republic of Korea.

The ADB Economics Working Paper Series presents data, information, and/or findings from ongoing research and studies to encourage exchange of ideas and to elicit comment and feedback about development issues in Asia and the Pacific. Since papers in this series are intended for quick and easy dissemination, the content may or may not be fully edited and may later be modified for final publication. 


\section{CONTENTS}

TABLES AND FIGURE

ABSTRACT

iv

$\begin{array}{ll}\text { I. INTRODUCTION } & 1\end{array}$

II. RELATED LITERATURE

III. DATA AND METHODS 5

$\begin{array}{ll}\text { IV. } & \text { FINDINGS }\end{array}$

$\begin{array}{ll}\text { V. CONCLUSION } & 31\end{array}$

$\begin{array}{ll}\text { APPENDIXES } & 33\end{array}$

$\begin{array}{ll}\text { REFERENCES } & 35\end{array}$ 


\section{TABLES AND FIGURE}

\section{TABLES}

1 Impact of VXO on Various Variables in Emerging Market Economies: Propensity Score

Weighted Regressions

1.1 Impact of $\mathrm{VXO}$ on Real Domestic Credit Growth 8

1.2 Impact of $\mathrm{VXO}$ on Real House Price Growth 10

1.3 Impact of $\bigvee X O$ on Real Stock Returns 11

1.4 Impact of VXO on the Change in the Loan-to-Deposit Ratio 12

1.5 Impact of $\mathrm{VXO}$ on Net Capital Flows 13

1.6 Impact of $\bigvee X O$ on Liability Flows 14

1.7 Impact of $\vee X O$ on Asset Flows

1.8 Impact of $\mathrm{VXO}$ on Foreign Direct Investment, Portfolio, and Other Investment 16 Liability Flows

1.9 Impact of VXO on Real Gross Domestic Product Growth 18

$2 \quad$ Impact of the Londono-Wilson Index on Various Variables in Emerging Market Economies 19

3 Impact of the United States Industrial Production Growth on Various Variables 21 in Emerging Market Economies

$4 \quad$ Impact of the Expected Probability of a United States Recession on Various Variables 23 in Emerging Market Economies

5 Impact of the United States Economic Policy Uncertainty Index on Various Variables 24 in Emerging Market Economies

6 Impact of the Federal Funds Rate on Various Variables in Emerging Market Economies 25

7 Impact of the Change in Total Assets of the Federal Reserve on Various Variables in 26 Emerging Market Economies

8 Impact of Non-United States Industrial Production Growth on Various Variables in 28

Emerging Market Economies

9 Impact of the Global Economic Policy Uncertainty Index on Various Variables in 29

Emerging Market Economies

10 Impact of the Expected Probability of Recessions Outside the United States on 30 Various Variables in Emerging Market Economies

A.1 Description and Sources for the Variables Used by Obstfeld, Ostry, and Qureshi 33

A.2 Description and Sources for the Variables Used by Londono and Wilson 34

\section{FIGURE}

Exchange Rate Regimes in Asian Countries 


\begin{abstract}
The insulating properties of flexible exchange rates have long been a highly contentious issue in emerging markets - not least in Asian emerging markets. A number of recent theoretical and empirical studies question whether a trade-off exists between rigid exchange rate regimes and insulation from foreign shocks when the degree of international capital mobility is high. On the other hand, Obstfeld, Ostry, and Qureshi (2017) find that countries with flexible exchange rate regimes experience less real and financial instability in the face of global financial volatility. We contribute to this empirical debate by significantly extending their analysis. Overall, our findings are broadly consistent with their results, suggesting that flexible exchange rate regimes are better at insulating emerging markets from external shocks. There are, however, a few subtle differences. In particular, we find somewhat less robust evidence that limited flexibility is enough to insulate emerging markets from shocks.
\end{abstract}

Keywords: exchange rate, exchange rate regime, fixed, flexible, insulate, intermediate, shock

JEL code: F31 


\section{INTRODUCTION}

The insulating properties of flexible exchange rates have long been a contentious issue in emerging markets - not least in Asian emerging markets. The debate goes back to the 1930s, when Japan-Asia's first emerging market - quickly recovered from the Great Depression by abandoning gold convertibility and depreciating the yen (Yasuba 1988). In the 1990s, Asia's emerging markets resisted international calls to move to greater exchange rate flexibility and experienced large financial inflows from abroad before being hit by an appreciating dollar-yen rate, higher oil prices, and a weakening global semiconductor market, resulting in the Asian financial crisis.

This experience accentuated calls for greater exchange rate flexibility in Asia and in emerging markets generally (Eichengreen 1999). Accordingly, many Asian economies moved in the direction of greater flexibility, although with notable exceptions, such as Hong Kong, China-and there may still be a gap between rhetoric and reality. According to Pontines and Rajan (2011), intervention in foreign exchange markets is extensive, suggesting that policy makers still have doubts about the insulating properties of full exchange rate flexibility, or at least they question whether the benefits in terms of insulation justify the costs. These costs can be reductions in exports and export-led growth if a competitively valued peg is sacrificed or financial fragility in the presence of currency mismatches on corporate, bank, and public balance sheets.

For some, these doubts are not entirely unfounded. The most recent theoretical and empirical literature questions whether a trade-off exists between exchange rate stability and insulation from foreign shocks in a setting of high international capital mobility. The traditional approach, grounded in the Mundell-Fleming model that posits the existence of this trade-off, is framed in terms of a trilemma (Mundell 1963). In this view, policy makers can attain only two of three desiderata: exchange rate stability, international capital mobility, and monetary autonomy. Here, it is the autonomy to alter domestic monetary policy that facilitates domestic adjustment and insulates against foreign shocks. The more recently observed global financial cycles which are characterized by large common movements in asset prices, gross flows, and leverage, have challenged this traditional view and argue for a policy dilemma rather than trilemma (Rey 2015, 2016). This posits a global financial cycle or disturbance that affects all countries, regardless of their exchange rate regime. Therefore, independent monetary policies are only possible if and only if the capital account is managed either directly or indirectly.

A second, related strand of recent research focuses on the international role of the dollar. This argues that exchange rate flexibility provides emerging markets with at best limited flexibility from shocks emanating from markets in dollar-denominated assets. Shin (2016) argues that dollardenominated credit is the dominant form of funding in the international interbank market, so that changes in interest rates in the United States (US) (and in euro-dollar markets) can strongly affect financial conditions in emerging markets regardless of their exchange rate regime. Gopinath (2017a, 2017b) emphasizes the prevalence of dollar pricing in international merchandise transactions, and the implication that nominal exchange rate changes will not translate into commensurate real exchange rate changes, thereby limiting the stabilization benefits of currency flexibility in developing countries.

This recent revisionism concerning the benefits of exchange rate flexibility has not gone unchallenged. Obstfeld, Ostry, and Qureshi (2017), in their major empirical analysis, marshal evidence of the stabilizing properties of flexible exchange rates. They study some 40 emerging markets over 19862013, distinguishing countries with fixed, intermediate, and flexible exchange rates, and consider the transmission of global financial shocks to domestic credit growth, house price growth, capital flows, and 
gross domestic product (GDP) growth, among other variables. Their results support the hypothesis that countries with fixed exchange rates experience greater real and financial instability and cyclicality because of global financial volatility. But they do not find that countries with intermediate exchange rate regimes with a limited degree of exchange rate flexibility are more susceptible to global financial volatility than countries with flexible exchange rates. Their conclusions suggest that even limited degrees of exchange rate flexibility can have useful insulation properties and thus support the approach of many Asian countries.

The inconclusive nature of this debate poses a dilemma for policy makers in Asia. Should they continue to move in the direction of exchange rate flexibility to enhance their insulation from global financial shocks? Or have the insulating properties of flexible exchange rates been oversold? There is no certain answer to either question because of the very different conclusions of the various studies.

This paper aims to shed further light on these issues by revisiting and extending Obstfeld, Ostry, and Qureshi (2017). Specifically, we consider the following extensions and sensitivity checks:

(i) We consider the robustness of the findings of Obstfeld, Ostry, and Qureshi (2017) to alternative data sources. As some of the authors' series are proprietary, we have to consider using different proprietary or publicly available data in our analysis. ${ }^{1}$ The comparison of our data sources to theirs is summarized in Appendix Table A.1.

(ii) We consider a wider variety of global financial shocks. Obstfeld, Ostry, and Qureshi (2017) consider the VXO (the precursor of the Chicago Board Options Exchange's Volatility Index, which is constructed using the implied volatility of a range of S\&P 500 index options). We also consider other measures of global volatility. These include, following Londono and Wilson (2018), a global volatility index calculated as the market-value-weighted average of the implied volatility of equity options for seven countries (France, Germany, Japan, the Netherlands, Switzerland, the United Kingdom, and the US). We also consider three categories of fundamental drivers of global shocks, also following the Londono-Wilson approach. The first category comprises three US economic and risk variables (industrial production growth, the expected probability of a US recession within the next quarter, and economic policy uncertainty). The second is a pair of US monetary policy shocks (changes in the federal funds rate and changes in Federal Reserve assets, the latter as a way of capturing unconventional monetary policies). The third is a pair of global factors not emanating from the US (non-US industrial production growth and the Global Economic Policy Uncertainty Index).

(iii) We also use an alternative empirical methodology designed to better control for the ways in which treatment-group countries (those with pegged exchange rates) and control-group countries (those with flexible exchange rate) differ. Ordinary regression methods may not be ideal for estimating the impact of alternative exchange rate regimes insofar as countries with different (observable and unobservable) characteristics are weighted equally. We instead use propensity score weighted regression methods; these are expressly designed to give more weight to comparable observations within treatment and control groups.

1 Most nonproprietary data are from Obstfeld, Ostry, and Qureshi (2017), and we greatly appreciate the authors sharing their data. 
Our findings are broadly consistent with Obstfeld, Ostry, and Qureshi (2017), although there are some important differences. First, using regression methods with, in some cases, alternative data sources we are largely able to replicate their findings on the contrast between countries with pegged and flexible exchange rates. ${ }^{2}$ For countries with pegged rates, there is evidence of a larger negative impact of the $\mathrm{VXO}$ on real domestic credit growth, real house price growth, the change in loan-to-deposit ratios, net capital flows, liability flows, and real GDP growth. Like Obstfeld, Ostry, and Qureshi (2017), we find less evidence of differences in sensitivity to this measure of global volatility in the contrast between countries with intermediate regimes of limited flexibility and countries with floating rates.

Second, we find these results again largely carry over when instead using propensity score methods, although with a few important differences. In particular, there is stronger evidence when using this approach that intermediate regimes also provide less insulation. For example, we find the VXO has a significantly larger negative effect on changes in the loan-to-deposit ratio and real GDP growth in countries with intermediate as opposed to flexible exchange rate regimes.

By applying propensity score weighted regression methods, we run the same equations as in Obstfeld, Ostry, and Qureshi (2017), but replace the VXO, which is US based, with a variant of the composite global volatility measure constructed in Londono and Wilson (2015) (Appendix Table A.2). We find evidence that both hard pegs and intermediate regimes heighten the sensitivity of real GDP growth and the change in the loan-to-deposit ratio to global volatility. Evidently, considering a wider geographical range of external volatility shocks shows that it is not only hard pegs that heighten vulnerability to external volatility relative to the flexible rate alternative.

Third, we find that flexible exchange rates provide differing degrees of insulation from different specific external disturbances. We use the eight fundamental variables making up the Londono-Wilson index, individually and in turn, as a measure of global shocks, and report the same coefficients, again using propensity score weighted regression methods.

The results suggest that less flexible exchange rates heighten the impact of fluctuations in US industrial production and of expectations of a US recession on financial variables, such as credit growth, house prices, and the change in loan-to-deposit ratios. But they do not heighten the likelihood of a domestic recession (or, more precisely, of a slowdown in real GDP growth). Strikingly, there is little support for the notion that more flexible exchange rates provide insulation from federal fund rate shocks. The absence of insulation may reflect the exceptional role of the dollar in interbank credit flows, irrespective of exchange rate regime, à la Shin. Or it may reflect the exceptional role of the dollar in trade invoicing, à la Gopinath. ${ }^{3}$ In contrast, an intermediate exchange rate regime heightens the impact of nonUS industrial production shocks on certain financial variables (domestic credit growth, the change in the loan-to-deposit ratio, net capital flows).

Thus, our findings are consistent with analyses that emphasize the ability of flexible exchange rates to provide insulation from dollar-based shocks, and not with studies that deny the existence of those insulating properties in general. Our findings are also consistent with the traditional trilemma framework, but with nuances. They are, however, less consistent with studies implying that limited

\footnotetext{
Regression results available on request.

3 Although the absence of an effect may also reflect the endogeneity of the Federal Reserve's policy for other US economic conditions that work in an offsetting direction.
} 
flexibility is enough if policy makers want to avoid accentuating the impact of external volatility on domestic conditions through their choice of exchange rate regime.

\section{RELATED LITERATURE}

We are not the first to consider the questions raised in the introduction. An extensive literature on the 1920s and 1930s, when countries experienced "the mother of all global shocks," supports the notions that pegged exchange rates encouraged procyclical capital flows (Accominotti and Eichengreen 2016), that flexible exchange rates provided insulation (Choudhri and Kochin 1980), and that domestic monetary autonomy was valuable for stabilizing economies in the volatile macroeconomic and financial circumstances of the period (Eichengreen and Sachs 1985, Eichengreen 1992).

With the shift from fixed to variable exchange rates after the collapse of the Bretton Woods system, a series of studies using more recent historical data sought to compare the behavior of a range of real and nominal variables under alternative regimes. Baxter and Stockman (1989), in an influential contribution, famously found little difference in the behavior of these variables across regimes, in stark contrast to findings for the earlier period.

Many of the authors who have revisited the findings of this work concluded that the impact of external real and financial shocks is in fact significantly greater under managed regimes compared with floats. Philippon, Zettelmeyer, and Borensztein (2001) analyzed the paired case studies of Hong Kong, China versus Singapore, and Argentina versus Mexico, finding some evidence that the pegged rate cases (Argentina and Hong Kong, China) were more susceptible to external shocks. Shambaugh (2004) and Frankel, Schmukler, and Serven (2004) used cross-country regressions to estimate the link between US and foreign interest rates; they found this link, indicative of transmission, is stronger in countries with pegged exchange rate regimes. Miniane and Rogers (2007), by estimating structural vector autoregressions, found that the transmission of US interest rate shocks is stronger in countries that shun capital controls and have pegged exchange rate regimes (although the estimated effect was not always significant). Aizenman, Chinn, and Ito (2015) documented that financial conditions in emerging markets are more tightly linked to those in the center countries when the emerging markets in question maintain pegged exchange rates. Bekaert and Mehl (2017) found evidence that interest rates in emerging markets continue to be less influenced by those in the US when exchange rates are flexible.

Other studies refined these analyses in various directions. Ricci and Shi (2016) emphasized heterogeneity among emerging markets, arguing that floating allows for policy independence, but more so for some countries than others. Bluedorn and Bowdler (2010) distinguished between anticipated and unanticipated and between exogenous and endogenous changes in US interest rates, and showed that interest rate links are especially strong across exchange rate regimes when the center-country shock is exogenous and unanticipated. Caceres, Carriere-Swallow, and Gruss (2016) distinguished between common shocks leading to business cycle synchronization and movements in domestic interest rates triggered by foreign monetary policy, finding that the second effect, known as monetary policy spillovers, is stronger when the exchange rate is fixed (and when the common shock is partialed out). Tovar (2004) and Georgiadis and Mehl (2015) added national balance sheets and the currency composition of foreign assets and liabilities, showing that the insulating properties of flexible exchange rates are weaker in the presence of net foreign currency liabilities (although those insulating properties still exist). Edwards (2015), focusing on three Latin American countries, concluded that the insulation provided by flexible exchange rates is at best partial, finding that the "degree of monetary policy independence is lower than what traditional models suggest." 
Thus, the literature provides mixed results on the stabilization properties of flexible exchange rates and the insulation they provide from global volatility shocks. And this is our motivation for revisiting the question.

\section{DATA AND METHODS}

Appendix Tables A.1 and A.2 describe the quarterly data from 1983 to 2013 for 43 emerging markets used in the analysis. Much of the data comes from the International Monetary Fund's International Financial Statistics. ${ }^{4}$ As measures of country conditions, we use the binary measures of the openness of the capital account of the balance of payments constructed by Quinn and Toyoda (2008). The index of institutional quality (average of 12 political risk components) comes from the Political Risk Group. ${ }^{5}$ Local share prices in real terms come from CEIC and our calculations. House prices in real terms are as calculated by the Bank for International Settlements. ${ }^{6}$

Like Obstfeld, Ostry, and Qureshi (2017), we considered alternative measures of exchange rate regimes, including Ghosh, Ostry, and Qureshi (2015) and Reinhart and Rogoff (2004), as updated by other authors. We choose Ghosh, Ostry, and Qureshi's (2015) classification. This is a measure of the de facto exchange rate regime, which differs in many cases from the official regime reported by governments to the International Monetary Fund, and presumably more relevant for economic outcomes.

The measure of exchange rate regime is defined in a way that the movements of actual exchange rates in a stricter regime are less volatile than those in a more flexible regime. ${ }^{7}$ This de facto regime classification suggests some lingering "fear of floating" in Asia and allows for more time variations relative to the de jure classification. Figure 1 shows that despite their de jure free floating of exchange rate regimes, India, Indonesia, and Thailand were pursuing a de facto controlled regime according to the classification. In the Philippines, the Republic of Korea, and Sri Lanka, authorities flip-flopped between de facto free floats and more controlled regimes, despite officially being free floaters.

To measure shocks, we used the VXO and VIX from Bloomberg, US 3-month Treasury bill rates, and 10-year government bond yields from the International Financial Statistics and Bloomberg. The actual federal funds rate is from International Financial Statistics and the shadow federal funds rate (where the shadow rate is constructed using the size of the central bank's balance sheet when the rate approaches the zero lower bound) is from the Federal Reserve Economic Data maintained by the Federal Reserve Bank of St. Louis. ${ }^{8}$

\footnotetext{
International Monetary Fund. International Financial Statistics. http://www.iif.com/ (accessed 18 August 2018). The Political Risk Group. http://www.prsgroup.com/ (accessed 18 August 2018).

Bank for International Settlements. https://www.bis.org/ (accessed 18 August 2018).

7 For the International Monetary Fund's classification of exchange rate regimes and monetary policy frameworks, see https://www.imf.org/external/np/mfd/er/2006/eng/0706.htm.

8 Federal Reserve Bank of St. Louis. Federal Reserve Economic Data. https//fred.stlouisfed.org (accessed 18 August 2018).
} 


\section{Exchange Rate Regimes in Asian Countries}

Indonesia

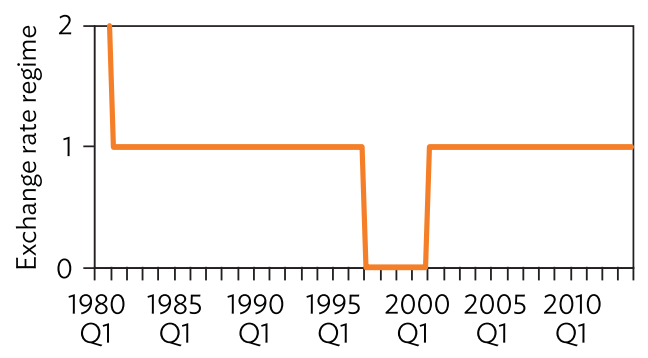

Malaysia

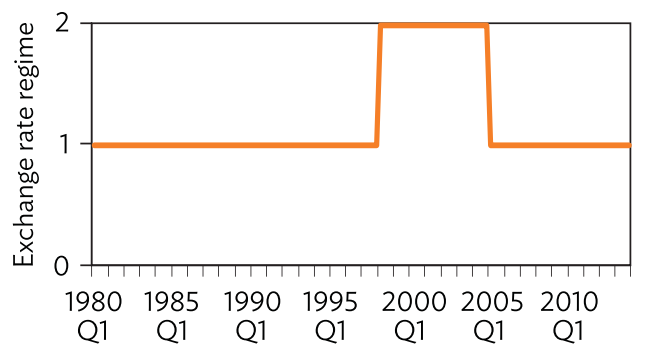

People's Republic of China

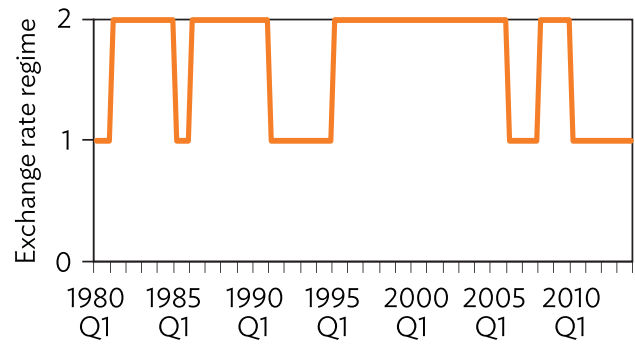

Sri Lanka

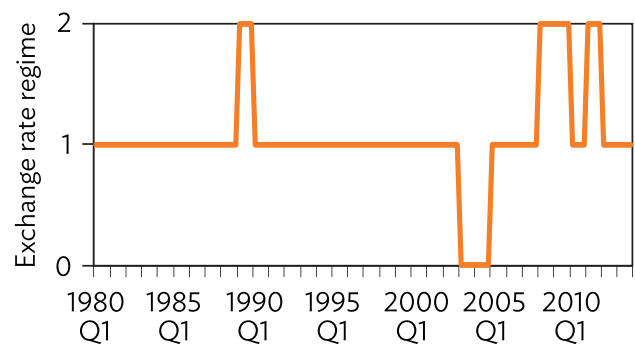

India

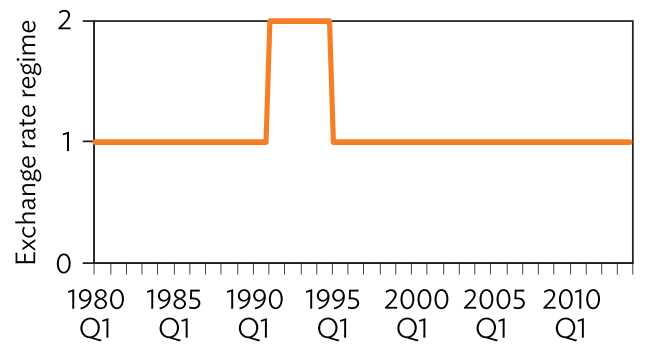

Philippines

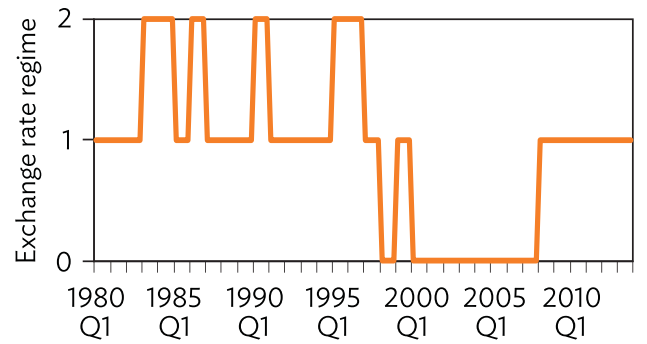

Republic of Korea

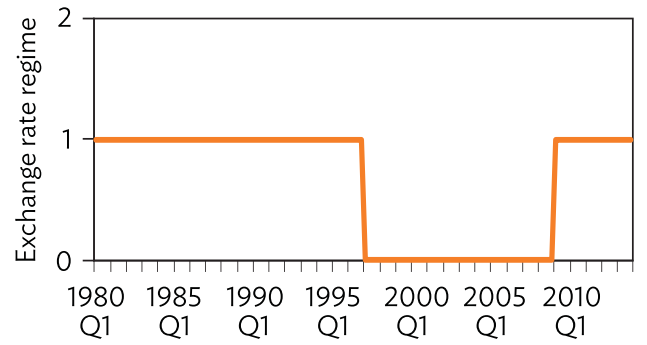

Thailand

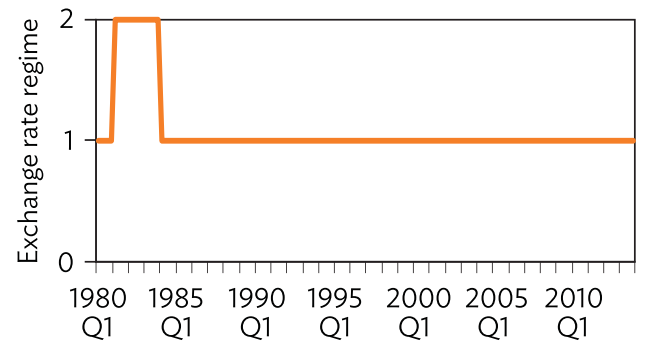

Notes:

1. The exchange rate regime data are collected from Maurice Obstfeld, Jonathan Ostry, and Mahvash Qureshi. 2017. A Tie That Binds: Revisiting the Trilemma in Emerging Market Economies. IMF Working Paper. WP/17/130. Washington, DC: International Monetary Fund. These data are based on Atish Ghosh, Jonathan Ostry, and Mahvesh Qureshi. 2015. Exchange Rate Management and Crisis Susceptibility: A Reassessment. IMF Economic Review. 63. pp. 238-76.

2. The free-floating, intermediate, and fixed exchange rate regimes are indicated by 0,1 , and 2 , respectively. Source: Authors. 
To calculate the Londono-Wilson index of external disturbances, we combined data for equityoption-implied volatility for seven countries: France, Germany, Japan, the Netherlands, Switzerland, the United Kingdom, and the US. These data are collected from Datastream, except for Japan and the US. Here, the data sources are from Osaka University's Center for the Study of Finance and Insurance and the Chicago Board Options Exchange. Alternatively, following Londono and Wilson, we considered also eight variables capturing different aspects of external shocks and volatility: US industrial production, the expected probability of a US recession, an index of US economic policy uncertainty, the federal funds rate, the change in Federal Reserve assets as a share of US GDP, non-US industrial production growth, a global economic policy uncertainty index, and the expected probability of recessions outside of the US. Appendix Table A.2 has a description and lists the sources of the variables used to construct the Londono-Wilson index, and gives further details on the eight variables.

We use both regression and propensity score methods. Propensity score methods are more appropriate when there are important differences between the treated and untreated observations (in our context, between countries with fixed and flexible exchange rates). Propensity score matching attempts to formally predict the likelihood of an observation (in our context, a country) receiving the treatment (choosing to peg its exchange rate) as a function of a set of covariates. This should reduce the bias due to confounding variables that could otherwise affect an estimate of the treatment effect from simply comparing outcomes among units that received the treatment versus those that did not. This approach is especially appropriate in cases where the treatment and control groups differ along multiple dimensions, since it permits a linear combination of covariates to be combined into a single score. It amounts to selecting or more heavily weighting the subset of units that are most similar to the treatment units.

We seek to estimate the impacts of not only the exchange rate regime but also their interaction with global shocks. Since we are interested in the coefficients of the interaction terms, which cannot be estimated simply by comparing the performance of the control group with that of the treatment group, we instead estimate propensity score weighted regressions. By weighting on the basis of propensity scores, these estimates give more weight to observations that are more likely to match, which should result in more reliable estimates.

\section{FINDINGS}

We were able to replicate the findings in Obstfeld, Ostry, and Qureshi (2017) using our dataset and their regression methods. ${ }^{9}$ The negative impact of the $\mathrm{VXO}$ is larger on real domestic credit growth, real house price growth, the change in loan-to-deposit ratios, net capital flows, liability flows, and real GDP growth in economies with pegged exchange rates, where free floating is the alternative. Like Obstfeld, Ostry, and Qureshi (2017), we find less evidence of differences in sensitivity to this measure of global volatility in the contrast between economies with intermediate regimes as opposed to those with floating rates.

The results for real house price growth are more complex and difficult to interpret than the others. This is because fixed rate regimes increase the sensitivity of price to the $\mathrm{VXO}$, but intermediate regimes significantly reduce that sensitivity relative to the omitted alternative of floating rates. Similarly, for asset flows, fixed rate regimes are almost equally sensitive to the $V X O$, while intermediate exchange regimes are less responsive to the $\mathrm{VXO}$ than floating exchange rates. Since the sum of the coefficients

9 Regressions available from the authors on request. 
of the VXO and its interaction with intermediate regimes is essentially zero, outflows of asset capital that are domestic-resident driven are least sensitive to the $\mathrm{VXO}$ under intermediate regimes.

The results were again similar when estimating these same relationships using propensity score weighted regressions. While propensity score matching methods are often used in the literature to estimate the impact of adopting different exchange rate regimes, it is not straightforward to apply the methodology to our case. ${ }^{10}$ We estimate the impacts of not only the exchange rate regime but also of its interactions with global shocks. We are especially interested in the coefficients on the interaction terms, which cannot be estimated by simply comparing the performance of the control group with that of the treatment group. As noted earlier, we adopt propensity score weighted regressions, which, by using propensity scores, give more weight to observations that are more likely to match.

Tables 1.1-1.9 report the results obtained from this alternative methodology following the specification in Tables 1-9 in Obstfeld, Ostry, and Qureshi (2017). The main results obtained from the two alternative methodologies are qualitatively very similar. Real stock returns are almost equally sensitive to the $\mathrm{VXO}$ in countries with fixed and intermediate regimes. The change in loan-to-deposit ratios falls more sharply in response to $\mathrm{VXO}$ shocks under both fixed and intermediate regimes (significantly so in columns 5-7 of Table 1.4). Asset flows appear to be least sensitive to VXO shocks under intermediate regimes, but there are also some notable differences. Unlike Obstfeld, Ostry, and Qureshi (2017), we find some evidence that the impact of the VXO on the growth of domestic credit and GDP is amplified not only under fixed exchange rate regimes but also under intermediate ones. This is somewhat stronger evidence that even limiting exchange rate flexibility, as opposed to eliminating it entirely, may significantly diminish the insulation from external shocks.

\section{Table 1: Impact of VXO on Various Variables in Emerging Market Economies: Propensity Score Weighted Regressions}

Table 1.1: Impact of VXO on Real Domestic Credit Growth

\begin{tabular}{|c|c|c|c|c|c|c|c|}
\hline & $\begin{array}{c}1986- \\
2013\end{array}$ & $\begin{array}{c}1986- \\
2013 \\
\end{array}$ & $\begin{array}{c}1986- \\
2013 \\
\end{array}$ & $\begin{array}{c}1986- \\
2013\end{array}$ & $\begin{array}{c}1986- \\
2013 \\
\end{array}$ & $\begin{array}{c}2000- \\
2013\end{array}$ & $\begin{array}{c}1986- \\
2013 \\
\end{array}$ \\
\hline Variables & $(1)$ & $(2)$ & (3) & (4) & (5) & (6) & $(7)$ \\
\hline Intermediate regime & $\begin{array}{c}1.391^{*} \\
(0.788)\end{array}$ & $\begin{array}{c}2.522 \\
(2.145)\end{array}$ & $\begin{array}{r}2.748 \\
(2.179)\end{array}$ & $\begin{array}{c}1.848 \\
(2.505)\end{array}$ & $\begin{array}{c}4.253^{*} \\
(2.275)\end{array}$ & $\begin{array}{l}5.006^{* *} \\
(2.113)\end{array}$ & $\begin{array}{c}2.969 \\
(2.636)\end{array}$ \\
\hline Fixed regime & $\begin{array}{l}2.995^{* * *} \\
(1.097)\end{array}$ & $\begin{array}{l}10.596^{* * *} \\
(3.429)\end{array}$ & $\begin{array}{l}10.484^{* * *} \\
(3.506)\end{array}$ & $\begin{array}{l}11.577^{* * *} \\
(4.096)\end{array}$ & $\begin{array}{l}11.718^{* * *} \\
(3.441)\end{array}$ & $\begin{array}{l}11.159^{* * *} \\
(3.740)\end{array}$ & $\begin{array}{l}11.937^{* *} \\
(4.590)\end{array}$ \\
\hline $\log (\mathrm{VXO})$ & $\begin{array}{l}-2.211^{* * *} \\
(0.474)\end{array}$ & $\begin{array}{l}-1.252^{* *} \\
(0.602)\end{array}$ & $\begin{array}{l}-1.218^{* *} \\
(0.592)\end{array}$ & $\begin{array}{c}-0.990 \\
(0.724)\end{array}$ & & & \\
\hline Intermediate $\times \log (\mathrm{VXO})$ & & $\begin{array}{c}-0.368 \\
(0.676)\end{array}$ & $\begin{array}{c}-0.444 \\
(0.679)\end{array}$ & $\begin{array}{l}-0.443 \\
(0.755)\end{array}$ & $\begin{array}{l}-0.817 \\
(0.736)\end{array}$ & $\begin{array}{l}-1.353^{* *} \\
(0.630)\end{array}$ & $\begin{array}{l}-1.034 \\
(0.805)\end{array}$ \\
\hline Fixed $\times \log (\mathrm{VXO})$ & & $\begin{array}{c}-2.532^{* *} \\
(1.007)\end{array}$ & $\begin{array}{c}-2.496^{* *} \\
(1.014)\end{array}$ & $\begin{array}{l}-2.827^{* *} \\
(1.095)\end{array}$ & $\begin{array}{l}-2.766^{* *} \\
(1.027)\end{array}$ & $\begin{array}{l}-3.032^{* *} \\
(1.153)\end{array}$ & $\begin{array}{c}-3.958^{* * *} \\
(1.384)\end{array}$ \\
\hline Lagged real GDP growth & $\begin{array}{l}0.935^{* * *} \\
(0.173)\end{array}$ & $\begin{array}{l}0.918^{* * *} \\
(0.169)\end{array}$ & $\begin{array}{l}0.913^{* * *} \\
(0.172)\end{array}$ & $\begin{array}{l}0.897^{* * *} \\
(0.172)\end{array}$ & $\begin{array}{l}0.880^{* * *} \\
(0.157)\end{array}$ & $\begin{array}{l}0.833^{* * *} \\
(0.252)\end{array}$ & $\begin{array}{l}1.149^{* * *} \\
(0.144)\end{array}$ \\
\hline
\end{tabular}

10 For example, Cushman and De Vita (2017) examine whether fixed exchange rates encourage more inward foreign direct investment by using propensity score matching methods. 
Table 1.1 continued

\begin{tabular}{|c|c|c|c|c|c|c|c|}
\hline & $\begin{array}{c}1986- \\
2013 \\
\end{array}$ & $\begin{array}{c}1986- \\
2013 \\
\end{array}$ & $\begin{array}{c}1986- \\
2013 \\
\end{array}$ & $\begin{array}{c}1986- \\
2013 \\
\end{array}$ & $\begin{array}{c}1986- \\
2013 \\
\end{array}$ & $\begin{array}{c}2000- \\
2013\end{array}$ & $\begin{array}{c}1986- \\
2013 \\
\end{array}$ \\
\hline Variables & $(1)$ & (2) & (3) & (4) & (5) & (6) & (7) \\
\hline Lagged private credit/GDP & $\begin{array}{l}-0.111^{* * *} \\
(0.013)\end{array}$ & $\begin{array}{l}-0.113^{* * *} \\
(0.014)\end{array}$ & $\begin{array}{l}-0.116^{* * *} \\
(0.016)\end{array}$ & $\begin{array}{l}-0.120^{* * *} \\
(0.017)\end{array}$ & $\begin{array}{l}-0.103^{* * *} \\
(0.013)\end{array}$ & $\begin{array}{l}-0.139^{* * *} \\
(0.016)\end{array}$ & $\begin{array}{l}-0.095^{* * *} \\
(0.021)\end{array}$ \\
\hline Real US T-bill rate & & & $\begin{array}{c}0.049 \\
(0.064)\end{array}$ & & & & \\
\hline Intermediate $\times$ real US T-bill rate & & & $\begin{array}{c}0.050 \\
(0.065)\end{array}$ & & & & \\
\hline Fixed $\times$ real US T-bill rate & & & $\begin{array}{l}-0.159 \\
(0.206)\end{array}$ & & & & \\
\hline Real shadow federal funds & & & & $\begin{array}{r}-0.006 \\
(0.153)\end{array}$ & & & \\
\hline Intermediate $\times$ real shadow rate & & & & $\begin{array}{c}0.321^{*} \\
(0.165)\end{array}$ & & & \\
\hline Fixed $\times$ real shadow rate & & & & $\begin{array}{l}-0.014 \\
(0.288)\end{array}$ & & & \\
\hline Lagged net capital flows/GDP & & & & & & & $\begin{array}{l}-0.001 \\
(0.007)\end{array}$ \\
\hline Lagged central bank policy rate & & & & & & & $\begin{array}{l}-0.326^{* * *} \\
(0.114)\end{array}$ \\
\hline Linear trend & $\begin{array}{l}0.029^{* *} \\
(0.014)\end{array}$ & $\begin{array}{l}0.030^{* *} \\
(0.014)\end{array}$ & $\begin{array}{l}0.032^{* *} \\
(0.013)\end{array}$ & $\begin{array}{l}0.043^{* *} \\
(0.016)\end{array}$ & & & \\
\hline Global financial crisis & $\begin{array}{l}2.632^{* * *} \\
(0.534)\end{array}$ & $\begin{array}{l}2.597^{* * *} \\
(0.503)\end{array}$ & $\begin{array}{l}2.556^{* * *} \\
(0.511)\end{array}$ & $\begin{array}{l}2.542^{* * *} \\
(0.501)\end{array}$ & & & \\
\hline Quarter-year effects & No & No & No & No & Yes & Yes & Yes \\
\hline Observations & 2,484 & 2,484 & 2,484 & 2,449 & 2,484 & 1,828 & 1,600 \\
\hline Adjusted $R^{2}$ & 0.260 & 0.267 & 0.270 & 0.279 & 0.309 & 0.497 & 0.572 \\
\hline No. of countries & 43 & 43 & 43 & 43 & 43 & 42 & 35 \\
\hline
\end{tabular}

GDP = gross domestic product, $U S=$ United States.

Notes:

1. This is identical to Table 1 in Obstfeld, Maurice, Jonathan Ostry, and Mahvash Qureshi. 2017. "A Tie That Binds: Revisiting the Trilemma in Emerging Market Economies." IMF Working Paper WP/17/130. Washington, DC: International Monetary Fund. The one exception is that we rely on an alternative methodology of propensity score weighted regressions.

2. The dependent variable is the three-quarter moving average of quarterly real domestic private sector credit growth rate (in percent) in emerging market economies.

3. VXO is the precursor of the Chicago Board Options Exchange's Volatility Index or VIX.

4. Numbers in parentheses are clustered standard errors (by country).

***, ${ }^{* *},{ }^{*}$ indicate statistical significance at the $1 \%, 5 \%$, and $10 \%$ levels, respectively.

Source: Authors' calculations. 
Table 1.2: Impact of VXO on Real House Price Growth

\begin{tabular}{|c|c|c|c|c|c|c|c|}
\hline & $\begin{array}{c}1986- \\
2013 \\
\end{array}$ & $\begin{array}{c}1986- \\
2013 \\
\end{array}$ & $\begin{array}{c}1986- \\
2013 \\
\end{array}$ & $\begin{array}{c}1986- \\
2013 \\
\end{array}$ & $\begin{array}{c}1986- \\
2013 \\
\end{array}$ & $\begin{array}{c}2000- \\
2013\end{array}$ & $\begin{array}{c}1986- \\
2013 \\
\end{array}$ \\
\hline Variables & (1) & (2) & (3) & (4) & (5) & (6) & (7) \\
\hline Intermediate regime & $\begin{array}{c}1.361^{* *} \\
(0.602)\end{array}$ & $\begin{array}{c}2.154 \\
(4.630)\end{array}$ & $\begin{array}{r}2.264 \\
(4.831)\end{array}$ & $\begin{array}{c}2.702 \\
(4.704)\end{array}$ & $\begin{array}{c}1.302 \\
(4.796)\end{array}$ & $\begin{array}{c}2.174 \\
(4.768)\end{array}$ & $\begin{array}{c}2.056 \\
(5.595)\end{array}$ \\
\hline Fixed regime & $\begin{array}{c}1.384 \\
(1.027)\end{array}$ & $\begin{array}{l}20.836^{* * *} \\
(5.493)\end{array}$ & $\begin{array}{l}20.990^{* * *} \\
(5.648)\end{array}$ & $\begin{array}{l}20.522^{* * *} \\
(6.230)\end{array}$ & $\begin{array}{l}18.186^{* * *} \\
(5.018)\end{array}$ & $\begin{array}{l}18.325^{* * *} \\
(5.048)\end{array}$ & $\begin{array}{l}19.817^{* * *} \\
(5.465)\end{array}$ \\
\hline $\log (V X O)$ & $\begin{array}{l}-2.327^{* * *} \\
(0.802)\end{array}$ & $\begin{array}{c}0.100 \\
(0.937)\end{array}$ & $\begin{array}{c}0.139 \\
(0.985)\end{array}$ & $\begin{array}{l}0.020 \\
(1.108)\end{array}$ & & & \\
\hline Intermediate $\times \log (\mathrm{VXO})$ & & $\begin{array}{r}-0.099 \\
(1.439)\end{array}$ & $\begin{array}{c}-0.133 \\
(1.499)\end{array}$ & $\begin{array}{c}-0.285 \\
(1.480)\end{array}$ & $\begin{array}{l}0.167 \\
(1.553)\end{array}$ & $\begin{array}{c}0.106 \\
(1.544)\end{array}$ & $\begin{array}{c}0.272 \\
(1.817)\end{array}$ \\
\hline Fixed $\times \log (\mathrm{VXO})$ & & $\begin{array}{c}-6.187^{* * *} \\
(1.652)\end{array}$ & $\begin{array}{c}-6.222^{* * *} \\
(1.711)\end{array}$ & $\begin{array}{c}-6.171^{* * *} \\
(1.830)\end{array}$ & $\begin{array}{c}-5.444^{* * *} \\
(1.618)\end{array}$ & $\begin{array}{c}-5.258^{* * *} \\
(1.624)\end{array}$ & $\begin{array}{l}-5.081^{* *} \\
(1.757)\end{array}$ \\
\hline Lagged real GDP growth & $\begin{array}{l}1.155^{* * *} \\
(0.246)\end{array}$ & $\begin{array}{l}1.014^{* * *} \\
(0.261)\end{array}$ & $\begin{array}{l}1.003^{* * *} \\
(0.265)\end{array}$ & $\begin{array}{l}1.028^{* * *} \\
(0.268)\end{array}$ & $\begin{array}{l}1.410^{* * *} \\
(0.158)\end{array}$ & $\begin{array}{l}1.419^{* * *} \\
(0.157)\end{array}$ & $\begin{array}{l}1.463^{* * *} \\
(0.301)\end{array}$ \\
\hline Lagged domestic credit growth & $\begin{array}{l}0.213^{* * *} \\
(0.066)\end{array}$ & $\begin{array}{c}0.162^{* *} \\
(0.076)\end{array}$ & $\begin{array}{c}0.163^{*} \\
(0.078)\end{array}$ & $\begin{array}{l}0.160^{* *} \\
(0.072)\end{array}$ & $\begin{array}{c}0.198^{* *} \\
(0.073)\end{array}$ & $\begin{array}{c}0.197^{* *} \\
(0.073)\end{array}$ & $\begin{array}{c}0.187^{* *} \\
(0.079)\end{array}$ \\
\hline Real US T-bill rate & & & $\begin{array}{l}-0.003 \\
(0.070)\end{array}$ & & & & \\
\hline Intermediate $\times$ real US T-bill rate & & & $\begin{array}{c}0.067 \\
(0.124)\end{array}$ & & & & \\
\hline Fixed $\times$ real US T-bill rate & & & $\begin{array}{c}0.027 \\
(0.068)\end{array}$ & & & & \\
\hline Real shadow federal funds & & & & $\begin{array}{c}-0.114 \\
(0.118)\end{array}$ & & & \\
\hline Intermediate $\times$ real shadow rate & & & & $\begin{array}{c}-0.003 \\
(0.289)\end{array}$ & & & \\
\hline Fixed $\times$ real shadow rate & & & & $\begin{array}{r}0.067 \\
(0.177)\end{array}$ & & & \\
\hline Lagged net capital flows/GDP & & & & & & & $\begin{array}{c}0.007 \\
(0.021)\end{array}$ \\
\hline Lagged central bank policy rate & & & & & & & $\begin{array}{r}0.063 \\
(0.103)\end{array}$ \\
\hline Linear trend & $\begin{array}{c}-0.029 \\
(0.018)\end{array}$ & $\begin{array}{c}-0.033 \\
(0.022)\end{array}$ & $\begin{array}{l}-0.031 \\
(0.024)\end{array}$ & $\begin{array}{l}-0.043 \\
(0.029)\end{array}$ & & & \\
\hline Global financial crisis & $\begin{array}{c}-1.452 \\
(1.182)\end{array}$ & $\begin{array}{c}-1.224 \\
(1.109)\end{array}$ & $\begin{array}{c}-1.256 \\
(1.154)\end{array}$ & $\begin{array}{c}-1.149 \\
(1.118)\end{array}$ & & & \\
\hline Quarter-year effects & No & No & No & No & Yes & Yes & Yes \\
\hline Observations & 579 & 579 & 579 & 562 & 579 & 566 & 497 \\
\hline Adjusted $R^{2}$ & 0.436 & 0.519 & 0.515 & 0.511 & 0.556 & 0.573 & 0.541 \\
\hline No. of countries & 18 & 18 & 18 & 18 & 18 & 18 & 17 \\
\hline
\end{tabular}

GDP = gross domestic product, US = United States.

Notes:

1. This is identical to Table 2 in Obstfeld, Maurice, Jonathan Ostry, and Mahvash Qureshi. 2017. "A Tie That Binds: Revisiting the Trilemma in Emerging Market Economies." IMF Working Paper WP/17/130. Washington, DC: International Monetary Fund. The one exception is that we rely on an alternative methodology of propensity score weighted regressions.

2. The dependent variable is the three-quarter moving average of quarterly real house price growth rate (in percent) in emerging market economies. 3. VXO is the precursor of the Chicago Board Options Exchange's Volatility Index or VIX.

4. Numbers in parentheses are clustered standard errors (by country).

$* * *, * *,{ }^{*}$ indicate statistical significance at the $1 \%, 5 \%$, and $10 \%$ levels, respectively.

Source: Authors' calculations. 
Table 1.3: Impact of VXO on Real Stock Returns

\begin{tabular}{|c|c|c|c|c|c|c|c|}
\hline & $\begin{array}{c}1986- \\
2013 \\
\end{array}$ & $\begin{array}{c}1986- \\
2013 \\
\end{array}$ & $\begin{array}{c}1986- \\
2013 \\
\end{array}$ & $\begin{array}{c}1986- \\
2013 \\
\end{array}$ & $\begin{array}{c}1986- \\
2013 \\
\end{array}$ & $\begin{array}{c}2000- \\
2013 \\
\end{array}$ & $\begin{array}{c}1986- \\
2013 \\
\end{array}$ \\
\hline Variables & $(1)$ & $(2)$ & (3) & (4) & $(5)$ & $(6)$ & $(7)$ \\
\hline Intermediate regime & $\begin{array}{c}0.054 \\
(0.776)\end{array}$ & $\begin{array}{l}-9.926 \\
(6.261)\end{array}$ & $\begin{array}{l}-11.631^{*} \\
(6.262)\end{array}$ & $\begin{array}{c}-11.332 \\
(6.902)\end{array}$ & $\begin{array}{c}0.717 \\
(6.553)\end{array}$ & $\begin{array}{l}-0.268 \\
(6.848)\end{array}$ & $\begin{array}{l}-0.922 \\
(6.742)\end{array}$ \\
\hline Fixed regime & $\begin{array}{r}-2.730^{*} \\
(1.593)\end{array}$ & $\begin{array}{c}1.387 \\
(7.360)\end{array}$ & $\begin{array}{c}1.036 \\
(7.111)\end{array}$ & $\begin{array}{c}2.801 \\
(7.776)\end{array}$ & $\begin{array}{c}7.328 \\
(6.324)\end{array}$ & $\begin{array}{c}5.201 \\
(6.479)\end{array}$ & $\begin{array}{c}5.936 \\
(7.413)\end{array}$ \\
\hline $\log (\mathrm{VXO})$ & $\begin{array}{l}-8.808^{* * *} \\
(0.869)\end{array}$ & $\begin{array}{l}-9.529^{* * *} \\
(1.183)\end{array}$ & $\begin{array}{c}-10.017^{* * *} \\
(1.119)\end{array}$ & $\begin{array}{c}-11.160^{* * *} \\
(1.144)\end{array}$ & & & \\
\hline Intermediate $\times \log (\mathrm{VXO})$ & & $\begin{array}{l}3.310 \\
(2.059)\end{array}$ & $\begin{array}{c}3.761^{*} \\
(2.067)\end{array}$ & $\begin{array}{c}3.724 \\
(2.229)\end{array}$ & $\begin{array}{c}0.623 \\
(2.095)\end{array}$ & $\begin{array}{c}1.120 \\
(2.167)\end{array}$ & $\begin{array}{r}1.304 \\
(2.131)\end{array}$ \\
\hline Fixed $\times \log (V X O)$ & & $\begin{array}{l}-1.357 \\
(2.441)\end{array}$ & $\begin{array}{l}-1.415 \\
(2.405)\end{array}$ & $\begin{array}{l}-1.598 \\
(2.441)\end{array}$ & $\begin{array}{l}-2.449 \\
(2.185)\end{array}$ & $\begin{array}{l}-1.584 \\
(2.141)\end{array}$ & $\begin{array}{l}-2.219 \\
(2.357)\end{array}$ \\
\hline Lagged real GDP growth & $\begin{array}{l}-1.540^{* * *} \\
(0.372)\end{array}$ & $\begin{array}{l}-1.569^{* * *} \\
(0.378)\end{array}$ & $\begin{array}{l}-1.313^{* * *} \\
(0.362)\end{array}$ & $\begin{array}{l}-1.308^{* * *} \\
(0.382)\end{array}$ & $\begin{array}{l}-0.018 \\
(0.226)\end{array}$ & $\begin{array}{l}-0.163 \\
(0.293)\end{array}$ & $\begin{array}{l}-0.063 \\
(0.330)\end{array}$ \\
\hline Lagged domestic credit growth & $\begin{array}{c}0.115 \\
(0.087)\end{array}$ & $\begin{array}{c}0.104 \\
(0.088)\end{array}$ & $\begin{array}{c}0.115 \\
(0.085)\end{array}$ & $\begin{array}{c}0.170^{*} \\
(0.095)\end{array}$ & $\begin{array}{c}0.049 \\
(0.074)\end{array}$ & $\begin{array}{l}-0.002 \\
(0.085)\end{array}$ & $\begin{array}{l}-0.019 \\
(0.107)\end{array}$ \\
\hline Real US T-bill rate & & & $\begin{array}{l}-0.766^{* * *} \\
(0.102)\end{array}$ & & & & \\
\hline Intermediate $\times$ real US T-bill rate & & & $\begin{array}{l}-0.324 \\
(0.196)\end{array}$ & & & & \\
\hline Fixed $\times$ real US T-bill rate & & & $\begin{array}{l}-0.485^{* *} \\
(0.180)\end{array}$ & & & & \\
\hline Real shadow federal funds & & & & $\begin{array}{l}-1.061^{* * *} \\
(0.181)\end{array}$ & & & \\
\hline Intermediate $\times$ real shadow rate & & & & $\begin{array}{l}-0.266 \\
(0.413)\end{array}$ & & & \\
\hline Fixed $\times$ real shadow rate & & & & $\begin{array}{l}-0.504 \\
(0.308)\end{array}$ & & & \\
\hline Lagged net capital flows/GDP & & & & & & & $\begin{array}{l}-0.119^{* * *} \\
(0.033)\end{array}$ \\
\hline Lagged central bank policy rate & & & & & & & $\begin{array}{l}-0.113 \\
(0.105)\end{array}$ \\
\hline Linear trend & $\begin{array}{l}-0.060^{* * *} \\
(0.018)\end{array}$ & $\begin{array}{l}-0.063^{* * *} \\
(0.018)\end{array}$ & $\begin{array}{l}-0.122^{* * *} \\
(0.018)\end{array}$ & $\begin{array}{l}-0.163^{* * *} \\
(0.034)\end{array}$ & & & \\
\hline Global financial crisis & $\begin{array}{l}-9.352^{* * *} \\
(2.372)\end{array}$ & $\begin{array}{l}-9.272^{* * *} \\
(2.383)\end{array}$ & $\begin{array}{l}-7.404^{* * *} \\
(2.307)\end{array}$ & $\begin{array}{l}-9.111^{* * *} \\
(2.367)\end{array}$ & & & \\
\hline Quarter-year effects & No & No & No & No & Yes & Yes & Yes \\
\hline Observations & 1,766 & 1,766 & 1,766 & 1,737 & 1,766 & 1,385 & 1,233 \\
\hline Adjusted $R^{2}$ & 0.204 & 0.208 & 0.270 & 0.249 & 0.555 & 0.589 & 0.591 \\
\hline No. of countries & 34 & 34 & 34 & 34 & 34 & 33 & 29 \\
\hline \multicolumn{8}{|c|}{$\begin{array}{l}\text { GDP = gross domestic product, US = United States. } \\
\text { Notes: } \\
\text { 1. This is identical to Table } 3 \text { in Obstfeld, Maurice, Jonathan Ostry, and Mahvash Qureshi. 2017. "A Tie That Binds: Revisiting the Trilemma in } \\
\text { Emerging Market Economies." IMF Working Paper WP/17/130. Washington, DC: International Monetary Fund. The one exception is that we } \\
\text { rely on an alternative methodology of propensity score weighted regressions. } \\
\text { 2. The dependent variable is the three-quarter moving average of quarterly real stock price growth rate (in percent) in emerging market economies. } \\
\text { 3. VXO is the precursor of the Chicago Board Options Exchange's Volatility Index or VIX. } \\
\text { 4. Numbers in parentheses are clustered standard errors (by country). } \\
* * * * * *, * * \text { indicate statistical significance at the } 1 \%, 5 \% \text {, and 10\% levels, respectively. } \\
\text { Source: Authors' calculations. }\end{array}$} \\
\hline
\end{tabular}


Table 1.4: Impact of VXO on the Change in the Loan-to-Deposit Ratio

\begin{tabular}{|c|c|c|c|c|c|c|c|}
\hline & $\begin{array}{c}1986- \\
2013 \\
\end{array}$ & $\begin{array}{c}1986- \\
2013\end{array}$ & $\begin{array}{c}1986- \\
2013 \\
\end{array}$ & $\begin{array}{c}1986- \\
2013 \\
\end{array}$ & $\begin{array}{c}1986- \\
2013\end{array}$ & $\begin{array}{c}2000- \\
2013\end{array}$ & $\begin{array}{c}1986- \\
2013 \\
\end{array}$ \\
\hline Variables & $(1)$ & $(2)$ & (3) & (4) & (5) & $(6)$ & (7) \\
\hline Intermediate regime & $\begin{array}{c}1.622^{* *} \\
(0.744)\end{array}$ & $\begin{array}{c}4.431 \\
(2.704)\end{array}$ & $\begin{array}{c}4.731^{*} \\
(2.655)\end{array}$ & $\begin{array}{c}4.354 \\
(2.706)\end{array}$ & $\begin{array}{c}5.721^{* *} \\
(2.361)\end{array}$ & $\begin{array}{l}4.973^{* *} \\
(2.446)\end{array}$ & $\begin{array}{c}3.318 \\
(2.411)\end{array}$ \\
\hline Fixed regime & $\begin{array}{l}4.332^{* *} \\
(1.724)\end{array}$ & $\begin{array}{l}8.384^{* *} \\
(3.134)\end{array}$ & $\begin{array}{l}8.676^{* * *} \\
(3.195)\end{array}$ & $\begin{array}{l}8.711^{* * *} \\
(2.872)\end{array}$ & $\begin{array}{l}7.780^{* * *} \\
(2.301)\end{array}$ & $\begin{array}{c}6.712^{* *} \\
(2.654)\end{array}$ & $\begin{array}{c}4.150 \\
(2.769)\end{array}$ \\
\hline $\log (V X O)$ & $\begin{array}{l}-0.704 \\
(0.433)\end{array}$ & $\begin{array}{c}0.002 \\
(0.604)\end{array}$ & $\begin{array}{c}0.095 \\
(0.564)\end{array}$ & $\begin{array}{c}0.422 \\
(0.567)\end{array}$ & & & \\
\hline Intermediate $\times \log (\mathrm{V} \times \mathrm{O})$ & & $\begin{array}{l}-0.927 \\
(0.816)\end{array}$ & $\begin{array}{l}-1.030 \\
(0.796)\end{array}$ & $\begin{array}{l}-1.121 \\
(0.800)\end{array}$ & $\begin{array}{l}-1.527^{* *} \\
(0.752)\end{array}$ & $\begin{array}{l}-1.652^{* *} \\
(0.658)\end{array}$ & $\begin{array}{l}-1.389^{*} \\
(0.690)\end{array}$ \\
\hline Fixed $\times \log (V X O)$ & & $\begin{array}{l}-1.343^{*} \\
(0.744)\end{array}$ & $\begin{array}{l}-1.436^{*} \\
(0.734)\end{array}$ & $\begin{array}{l}-1.640^{* *} \\
(0.721)\end{array}$ & $\begin{array}{l}-1.668^{* *} \\
(0.685)\end{array}$ & $\begin{array}{l}-1.738^{* *} \\
(0.690)\end{array}$ & $\begin{array}{l}-1.569^{*} \\
(0.783)\end{array}$ \\
\hline Lagged real GDP growth & $\begin{array}{l}0.531^{* * *} \\
(0.141)\end{array}$ & $\begin{array}{l}0.527^{* * *} \\
(0.144)\end{array}$ & $\begin{array}{l}0.519^{* * *} \\
(0.149)\end{array}$ & $\begin{array}{l}0.476^{* * *} \\
(0.150)\end{array}$ & $\begin{array}{l}0.450^{* * *} \\
(0.141)\end{array}$ & $\begin{array}{c}0.366^{*} \\
(0.199)\end{array}$ & $\begin{array}{l}0.512^{* * *} \\
(0.153)\end{array}$ \\
\hline Lagged LTD ratio & $\begin{array}{l}-0.065^{* * *} \\
(0.013)\end{array}$ & $\begin{array}{l}-0.066^{* * *} \\
(0.014)\end{array}$ & $\begin{array}{l}-0.066^{* * *} \\
(0.013)\end{array}$ & $\begin{array}{l}-0.070^{* * *} \\
(0.013)\end{array}$ & $\begin{array}{l}-0.065^{* * *} \\
(0.011)\end{array}$ & $\begin{array}{l}-0.085^{* * *} \\
(0.018)\end{array}$ & $\begin{array}{l}-0.058^{* * *} \\
(0.017)\end{array}$ \\
\hline Real US T-bill rate & & & $\begin{array}{l}-0.008 \\
(0.066)\end{array}$ & & & & \\
\hline Intermediate $\times$ real US T-bill rate & & & $\begin{array}{c}0.086 \\
(0.075)\end{array}$ & & & & \\
\hline Fixed $\times$ real US T-bill rate & & & $\begin{array}{c}0.044 \\
(0.160)\end{array}$ & & & & \\
\hline Real shadow federal funds & & & & $\begin{array}{c}0.081 \\
(0.107)\end{array}$ & & & \\
\hline Intermediate $\times$ real shadow rate & & & & $\begin{array}{c}0.251^{*} \\
(0.140)\end{array}$ & & & \\
\hline Fixed $\times$ real shadow rate & & & & $\begin{array}{c}0.226 \\
(0.288)\end{array}$ & & & \\
\hline Lagged net capital flows/GDP & & & & & & & $\begin{array}{c}0.001 \\
(0.018)\end{array}$ \\
\hline Lagged central bank policy rate & & & & & & & $\begin{array}{l}-0.158^{* * *} \\
(0.037)\end{array}$ \\
\hline Linear trend & $\begin{array}{c}0.010 \\
(0.007)\end{array}$ & $\begin{array}{c}0.010 \\
(0.007)\end{array}$ & $\begin{array}{c}0.013^{*} \\
(0.007)\end{array}$ & $\begin{array}{l}0.032^{* * *} \\
(0.009)\end{array}$ & & & \\
\hline Global financial crisis & $\begin{array}{l}1.667^{* * *} \\
(0.613)\end{array}$ & $\begin{array}{l}1.651^{* * *} \\
(0.599)\end{array}$ & $\begin{array}{l}1.585^{* *} \\
(0.614)\end{array}$ & $\begin{array}{l}1.639^{* * *} \\
(0.582)\end{array}$ & & & \\
\hline Quarter-year effects & No & No & No & No & Yes & Yes & Yes \\
\hline Observations & 2,484 & 2,484 & 2,484 & 2,449 & 2,484 & 1,828 & 1,600 \\
\hline Adjusted $R^{2}$ & 0.193 & 0.195 & 0.196 & 0.215 & 0.369 & 0.325 & 0.336 \\
\hline No. of countries & 43 & 43 & 43 & 43 & 43 & 42 & 35 \\
\hline
\end{tabular}

GDP = gross domestic product, LTD = loan-to-deposit, US = United States.

Notes: This is identical to Table 4 in Obstfeld, Maurice, Jonathan Ostry, and Mahvash Qureshi. 2017. "A Tie That Binds: Revisiting the Trilemma in Emerging Market Economies." IMF Working Paper WP/17/130. Washington, DC: International Monetary Fund. The one exception is that we rely on an alternative methodology of propensity score weighted regressions.

2. The dependent variable is the three-quarter moving average of change in the loan-to-deposit ratio in emerging market economies.

3. VXO is the precursor of the Chicago Board Options Exchange's Volatility Index or VIX.

4. Numbers in parentheses are clustered standard errors (by country).

$* * * * *{ }^{*}$ indicate statistical significance at the $1 \%, 5 \%$, and $10 \%$ levels, respectively.

Source: Authors' calculations. 
Table 1.5: Impact of VXO on Net Capital Flows

\begin{tabular}{|c|c|c|c|c|c|c|}
\hline & $1986-2013$ & $1986-2013$ & $1986-2013$ & $1986-2013$ & $1986-2013$ & $2000-2013$ \\
\hline Variables & $(1)$ & $(2)$ & (3) & (4) & (5) & (6) \\
\hline Intermediate regime & $\begin{array}{c}1.726^{* *} \\
(0.657)\end{array}$ & $\begin{array}{r}5.853 \\
(3.717)\end{array}$ & $\begin{array}{c}5.925 \\
(3.755)\end{array}$ & $\begin{array}{c}6.469^{*} \\
(3.444)\end{array}$ & $\begin{array}{c}7.124^{*} \\
(3.587)\end{array}$ & $\begin{array}{c}8.375^{* *} \\
(3.566)\end{array}$ \\
\hline Fixed regime & $\begin{array}{l}1.184 \\
(1.994)\end{array}$ & $\begin{array}{l}15.231^{* *} \\
(5.960)\end{array}$ & $\begin{array}{l}15.338^{* *} \\
(5.945)\end{array}$ & $\begin{array}{l}14.693^{* *} \\
(5.435)\end{array}$ & $\begin{array}{l}15.942^{* *} \\
(5.995)\end{array}$ & $\begin{array}{l}16.714^{* * *} \\
(5.696)\end{array}$ \\
\hline $\log (V X O)$ & $\begin{array}{l}-1.832^{* * *} \\
(0.656)\end{array}$ & $\begin{array}{c}0.012 \\
(0.844)\end{array}$ & $\begin{array}{c}0.054 \\
(0.857)\end{array}$ & $\begin{array}{c}0.406 \\
(0.831)\end{array}$ & & \\
\hline Intermediate $\times \log (\mathrm{V} \times \mathrm{O})$ & & $\begin{array}{l}-1.343 \\
(1.177)\end{array}$ & $\begin{array}{l}-1.378 \\
(1.194)\end{array}$ & $\begin{array}{l}-1.678 \\
(1.123)\end{array}$ & $\begin{array}{l}-1.616 \\
(1.155)\end{array}$ & $\begin{array}{l}-1.975 \\
(1.208)\end{array}$ \\
\hline Fixed $\times \log (V X O)$ & & $\begin{array}{c}-4.664^{* *} \\
(1.758)\end{array}$ & $\begin{array}{c}-4.706^{* *} \\
(1.761)\end{array}$ & $\begin{array}{l}-4.545^{* * *} \\
(1.667)\end{array}$ & $\begin{array}{c}-4.622^{* *} \\
(1.760)\end{array}$ & $\begin{array}{c}-4.493^{* *} \\
(1.660)\end{array}$ \\
\hline Lagged real GDP growth & $\begin{array}{l}0.331^{* * *} \\
(0.109)\end{array}$ & $\begin{array}{l}0.315^{* * *} \\
(0.105)\end{array}$ & $\begin{array}{l}0.313^{* * *} \\
(0.106)\end{array}$ & $\begin{array}{l}0.288^{* * *} \\
(0.102)\end{array}$ & $\begin{array}{l}0.319^{* * *} \\
(0.115)\end{array}$ & $\begin{array}{c}0.393^{*} \\
(0.204)\end{array}$ \\
\hline Lagged institutional quality & $\begin{array}{l}20.900^{* * *} \\
(5.530)\end{array}$ & $\begin{array}{l}22.387^{* * *} \\
(5.495)\end{array}$ & $\begin{array}{l}21.992^{* * *} \\
(5.352)\end{array}$ & $\begin{array}{l}21.928^{* * *} \\
(5.587)\end{array}$ & $\begin{array}{l}15.900^{* *} \\
(6.016)\end{array}$ & $\begin{array}{l}20.027^{* *} \\
(9.729)\end{array}$ \\
\hline Lagged domestic credit/GDP & $\begin{array}{l}-0.039 \\
(0.025)\end{array}$ & $\begin{array}{l}-0.047^{*} \\
(0.024)\end{array}$ & $\begin{array}{c}-0.047^{*} \\
(0.024)\end{array}$ & $\begin{array}{l}-0.053^{* *} \\
(0.025)\end{array}$ & $\begin{array}{c}-0.048^{*} \\
(0.026)\end{array}$ & $\begin{array}{l}-0.089^{* * *} \\
(0.027)\end{array}$ \\
\hline Real US T-bill rate & & & $\begin{array}{c}0.012 \\
(0.086)\end{array}$ & & & \\
\hline Intermediate $\times$ real US T-bill rate & & & $\begin{array}{c}0.034 \\
(0.119)\end{array}$ & & & \\
\hline Fixed $\times$ real US T-bill rate & & & $\begin{array}{c}0.165 \\
(0.245)\end{array}$ & & & \\
\hline Real shadow federal funds & & & & $\begin{array}{c}0.170 \\
(0.173)\end{array}$ & & \\
\hline Intermediate $\times$ real shadow rate & & & & $\begin{array}{c}0.177 \\
(0.209)\end{array}$ & & \\
\hline Fixed $\times$ real shadow rate & & & & $\begin{array}{c}0.144 \\
(0.481)\end{array}$ & & \\
\hline Linear trend & $\begin{array}{r}0.000 \\
(0.017)\end{array}$ & $\begin{array}{c}0.002 \\
(0.016)\end{array}$ & $\begin{array}{r}0.006 \\
(0.018)\end{array}$ & $\begin{array}{c}0.028 \\
(0.027)\end{array}$ & & \\
\hline Global financial crisis & $\begin{array}{l}-1.439 \\
(0.939)\end{array}$ & $\begin{array}{l}-1.322 \\
(0.982)\end{array}$ & $\begin{array}{l}-1.458 \\
(0.977)\end{array}$ & $\begin{array}{l}-1.334 \\
(0.963)\end{array}$ & & \\
\hline Quarter-year effects & No & No & No & No & Yes & Yes \\
\hline Observations & 2,083 & 2,083 & 2,083 & 2,051 & 2,083 & 1,629 \\
\hline Adjusted $R^{2}$ & 0.380 & 0.391 & 0.390 & 0.392 & 0.436 & 0.474 \\
\hline No. of countries & 38 & 38 & 38 & 38 & 38 & 38 \\
\hline
\end{tabular}

GDP = gross domestic product, US = United States

Notes:

1. This is identical to Table 5 in Obstfeld, Maurice, Jonathan Ostry, and Mahvash Qureshi. 2017. "A Tie That Binds: Revisiting the Trilemma in Emerging Market Economies." IMF Working Paper WP/17/130. Washington, DC: International Monetary Fund. The one exception is that we rely on an alternative methodology of propensity score weighted regressions.

2. The dependent variable is the three-quarter moving average of quarterly net capital flows (in percent of GDP) in emerging market economies.

3. VXO is the precursor of the Chicago Board Options Exchange's Volatility Index or VIX.

4. Numbers in parentheses are clustered standard errors (by country).

***, **, ${ }^{*}$ indicate statistical significance at the $1 \%, 5 \%$, and $10 \%$ levels, respectively.

Source: Authors' calculations. 
Table 1.6: Impact of VXO on Liability Flows

\begin{tabular}{|c|c|c|c|c|c|c|}
\hline & 1986-2013 & $1986-2013$ & 1986-2013 & 1986-2013 & 1986-2013 & 2000-2013 \\
\hline Variables & (1) & (2) & (3) & (4) & (5) & (6) \\
\hline Intermediate regime & $\begin{array}{c}0.472 \\
(0.627)\end{array}$ & $\begin{array}{l}-3.228 \\
(3.243)\end{array}$ & $\begin{array}{c}-2.813 \\
(3.391)\end{array}$ & $\begin{array}{l}-1.863 \\
(3.054)\end{array}$ & $\begin{array}{c}1.979 \\
(4.232)\end{array}$ & $\begin{array}{c}1.781 \\
(4.831)\end{array}$ \\
\hline Fixed regime & $\begin{array}{c}1.559 \\
(1.634)\end{array}$ & $\begin{array}{l}13.894^{* *} \\
(6.304)\end{array}$ & $\begin{array}{l}14.144^{* *} \\
(6.296)\end{array}$ & $\begin{array}{l}13.756^{* *} \\
(5.813)\end{array}$ & $\begin{array}{l}17.506^{* *} \\
(7.150)\end{array}$ & $\begin{array}{l}17.441^{* *} \\
(7.124)\end{array}$ \\
\hline $\log (\mathrm{VXO})$ & $\begin{array}{l}-4.497^{* * *} \\
(0.864)\end{array}$ & $\begin{array}{l}-3.568^{* * *} \\
(0.787)\end{array}$ & $\begin{array}{l}-3.426^{* * *} \\
(0.770)\end{array}$ & $\begin{array}{l}-2.655^{* * *} \\
(0.850)\end{array}$ & & \\
\hline Intermediate $\times \log (\mathrm{VXO})$ & & $\begin{array}{c}1.253 \\
(1.055)\end{array}$ & $\begin{array}{c}1.090 \\
(1.115)\end{array}$ & $\begin{array}{l}0.554 \\
(1.098)\end{array}$ & $\begin{array}{c}0.026 \\
(1.291)\end{array}$ & $\begin{array}{c}0.234 \\
(1.455)\end{array}$ \\
\hline Fixed $\times \log (\mathrm{V} X \mathrm{O})$ & & $\begin{array}{l}-4.103^{*} \\
(2.042)\end{array}$ & $\begin{array}{l}-4.195^{* *} \\
(2.047)\end{array}$ & $\begin{array}{c}-4.160^{* *} \\
(1.966)\end{array}$ & $\begin{array}{l}-4.581^{* *} \\
(2.226)\end{array}$ & $\begin{array}{l}-3.966^{*} \\
(2.181)\end{array}$ \\
\hline Lagged real GDP growth & $\begin{array}{l}0.385^{* * *} \\
(0.121)\end{array}$ & $\begin{array}{l}0.369^{* * *} \\
(0.115)\end{array}$ & $\begin{array}{l}0.366^{* * *} \\
(0.116)\end{array}$ & $\begin{array}{l}0.314^{* * *} \\
(0.110)\end{array}$ & $\begin{array}{l}0.332^{* * *} \\
(0.119)\end{array}$ & $\begin{array}{c}0.407^{*} \\
(0.212)\end{array}$ \\
\hline Lagged institutional quality & $\begin{array}{l}24.804^{* * *} \\
(5.474)\end{array}$ & $\begin{array}{l}26.040^{* * *} \\
(5.541)\end{array}$ & $\begin{array}{l}25.307^{* * *} \\
(5.517)\end{array}$ & $\begin{array}{l}24.331^{* * *} \\
(5.767)\end{array}$ & $\begin{array}{l}18.016^{* * *} \\
(6.597)\end{array}$ & $\begin{array}{l}23.860^{* *} \\
(9.679)\end{array}$ \\
\hline Lagged domestic credit/GDP & $\begin{array}{l}-0.009 \\
(0.026)\end{array}$ & $\begin{array}{l}-0.013 \\
(0.024)\end{array}$ & $\begin{array}{l}-0.012 \\
(0.024)\end{array}$ & $\begin{array}{l}-0.020 \\
(0.026)\end{array}$ & $\begin{array}{l}-0.008 \\
(0.026)\end{array}$ & $\begin{array}{l}-0.027 \\
(0.028)\end{array}$ \\
\hline Real US T-bill rate & & & $\begin{array}{r}0.000 \\
(0.136)\end{array}$ & & & \\
\hline Intermediate $\times$ real US T-bill rate & & & $\begin{array}{c}0.145 \\
(0.216)\end{array}$ & & & \\
\hline Fixed $\times$ real US T-bill rate & & & $\begin{array}{c}0.256 \\
(0.275)\end{array}$ & & & \\
\hline Real shadow federal funds & & & & $\begin{array}{c}0.486^{* *} \\
(0.180)\end{array}$ & & \\
\hline Intermediate $\times$ real shadow rate & & & & $\begin{array}{c}0.337 \\
(0.360)\end{array}$ & & \\
\hline Fixed $\times$ real shadow rate & & & & $\begin{array}{c}0.270 \\
(0.506)\end{array}$ & & \\
\hline Linear trend & $\begin{array}{c}-0.021 \\
(0.018)\end{array}$ & $\begin{array}{r}-0.022 \\
(0.017)\end{array}$ & $\begin{array}{c}-0.013 \\
(0.017)\end{array}$ & $\begin{array}{c}0.040 \\
(0.029)\end{array}$ & & \\
\hline Global financial crisis & $\begin{array}{l}-3.243^{* *} \\
(1.541)\end{array}$ & $\begin{array}{c}-3.270^{*} \\
(1.617)\end{array}$ & $\begin{array}{c}-3.503^{* *} \\
(1.589)\end{array}$ & $\begin{array}{c}-3.160^{*} \\
(1.634)\end{array}$ & & \\
\hline Quarter-year effects & No & No & No & No & Yes & Yes \\
\hline Observations & 2,083 & 2,083 & 2,083 & 2,051 & 2,083 & 1,629 \\
\hline Adjusted $R^{2}$ & 0.392 & 0.402 & 0.402 & 0.413 & 0.467 & 0.501 \\
\hline No. of countries & 38 & 38 & 38 & 38 & 38 & 38 \\
\hline
\end{tabular}

GDP = gross domestic product, US = United States .

Notes:

1. This is identical to Table 6 in Obstfeld, Maurice, Jonathan Ostry, and Mahvash Qureshi. 2017. "A Tie That Binds: Revisiting the Trilemma in Emerging Market Economies." IMF Working Paper WP/17/130. Washington, DC: International Monetary Fund. The one exception is that we rely on an alternative methodology of propensity score weighted regressions.

2. The dependent variable is the three-quarter moving average of quarterly liability flows (in percent of GDP) in emerging market economies. 3. VXO is the precursor of the Chicago Board Options Exchange's Volatility Index or VIX.

4. Numbers in parentheses are clustered standard errors (by country).

${ }^{* * *}, * *,{ }^{*}$ indicate statistical significance at the $1 \%, 5 \%$, and $10 \%$ levels, respectively.

Source: Authors' calculations. 
Table 1.7: Impact of VXO on Asset Flows

\begin{tabular}{|c|c|c|c|c|c|c|}
\hline & $1986-2013$ & $1986-2013$ & $1986-2013$ & $1986-2013$ & $1986-2013$ & $2000-2013$ \\
\hline Variables & (1) & (2) & (3) & (4) & $(5)$ & (6) \\
\hline Intermediate regime & $\begin{array}{l}1.247^{* *} \\
(0.559)\end{array}$ & $\begin{array}{l}9.122^{* * *} \\
(2.673)\end{array}$ & $\begin{array}{l}8.789^{* * *} \\
(2.862)\end{array}$ & $\begin{array}{l}8.364^{* * *} \\
(2.792)\end{array}$ & $\begin{array}{c}5.156 \\
(3.400)\end{array}$ & $\begin{array}{c}6.624^{*} \\
(3.593)\end{array}$ \\
\hline Fixed regime & $\begin{array}{l}-0.319 \\
(0.719)\end{array}$ & $\begin{array}{c}1.366 \\
(3.685)\end{array}$ & $\begin{array}{c}1.221 \\
(3.659)\end{array}$ & $\begin{array}{c}0.996 \\
(3.930)\end{array}$ & $\begin{array}{l}-1.534 \\
(3.907)\end{array}$ & $\begin{array}{l}-0.626 \\
(4.262)\end{array}$ \\
\hline $\log (V X O)$ & $\begin{array}{l}2.660^{* * *} \\
(0.459)\end{array}$ & $\begin{array}{l}3.583^{* * *} \\
(0.567)\end{array}$ & $\begin{array}{l}3.485^{* * *} \\
(0.541)\end{array}$ & $\begin{array}{l}3.066^{* * *} \\
(0.631)\end{array}$ & & \\
\hline Intermediate $\times \log (\mathrm{VXO})$ & & $\begin{array}{l}-2.612^{* * *} \\
(0.865)\end{array}$ & $\begin{array}{l}-2.488^{* *} \\
(0.927)\end{array}$ & $\begin{array}{l}-2.249^{* *} \\
(0.968)\end{array}$ & $\begin{array}{l}-1.651 \\
(1.050)\end{array}$ & $\begin{array}{c}-2.220^{* *} \\
(1.059)\end{array}$ \\
\hline Fixed $\times \log (V X O)$ & & $\begin{array}{c}-0.553 \\
(1.221)\end{array}$ & $\begin{array}{c}-0.501 \\
(1.206)\end{array}$ & $\begin{array}{c}-0.378 \\
(1.243)\end{array}$ & $\begin{array}{c}-0.022 \\
(1.278)\end{array}$ & $\begin{array}{c}-0.510 \\
(1.292)\end{array}$ \\
\hline Lagged real GDP growth & $\begin{array}{l}-0.055^{*} \\
(0.031)\end{array}$ & $\begin{array}{l}-0.054^{*} \\
(0.030)\end{array}$ & $\begin{array}{l}-0.053^{*} \\
(0.029)\end{array}$ & $\begin{array}{l}-0.027 \\
(0.026)\end{array}$ & $\begin{array}{l}-0.012 \\
(0.026)\end{array}$ & $\begin{array}{l}-0.013 \\
(0.039)\end{array}$ \\
\hline Lagged institutional quality & $\begin{array}{l}-3.757 \\
(3.609)\end{array}$ & $\begin{array}{l}-3.502 \\
(3.544)\end{array}$ & $\begin{array}{l}-3.160 \\
(3.590)\end{array}$ & $\begin{array}{l}-2.231 \\
(3.544)\end{array}$ & $\begin{array}{l}-1.927 \\
(3.911)\end{array}$ & $\begin{array}{l}-3.513 \\
(6.102)\end{array}$ \\
\hline Lagged domestic credit/GDP & $\begin{array}{l}-0.031 \\
(0.023)\end{array}$ & $\begin{array}{l}-0.034 \\
(0.022)\end{array}$ & $\begin{array}{l}-0.035 \\
(0.022)\end{array}$ & $\begin{array}{l}-0.033 \\
(0.023)\end{array}$ & $\begin{array}{l}-0.040^{*} \\
(0.023)\end{array}$ & $\begin{array}{l}-0.062^{* *} \\
(0.023)\end{array}$ \\
\hline Real US T-bill rate & & & $\begin{array}{c}0.012 \\
(0.100)\end{array}$ & & & \\
\hline Intermediate $\times$ real US T-bill rate & & & $\begin{array}{l}-0.109 \\
(0.157)\end{array}$ & & & \\
\hline Fixed $\times$ real US T-bill rate & & & $\begin{array}{l}-0.094 \\
(0.124)\end{array}$ & & & \\
\hline Real shadow federal funds & & & & $\begin{array}{l}-0.315^{*} \\
(0.166)\end{array}$ & & \\
\hline Intermediate $\times$ real shadow rate & & & & $\begin{array}{l}-0.155 \\
(0.305)\end{array}$ & & \\
\hline Fixed $\times$ real shadow rate & & & & $\begin{array}{l}-0.131 \\
(0.197)\end{array}$ & & \\
\hline Linear trend & $\begin{array}{c}0.021 \\
(0.015)\end{array}$ & $\begin{array}{c}0.024 \\
(0.014)\end{array}$ & $\begin{array}{c}0.020 \\
(0.014)\end{array}$ & $\begin{array}{l}-0.012 \\
(0.022)\end{array}$ & & \\
\hline Global financial crisis & $\begin{array}{c}1.803 \\
(1.202)\end{array}$ & $\begin{array}{c}1.936 \\
(1.165)\end{array}$ & $\begin{array}{l}2.031^{*} \\
(1.139)\end{array}$ & $\begin{array}{l}1.816 \\
(1.140)\end{array}$ & & \\
\hline Quarter-year effects & No & No & No & No & Yes & Yes \\
\hline Observations & 2,103 & 2,103 & 2,103 & 2,070 & 2,103 & 1,649 \\
\hline Adjusted $R^{2}$ & 0.313 & 0.319 & 0.320 & 0.326 & 0.347 & 0.377 \\
\hline No. of countries & 39 & 39 & 39 & 39 & 39 & 39 \\
\hline
\end{tabular}

GDP = gross domestic product, $U S=$ United States .

Notes:

1. This is identical to Table 7 in Obstfeld, Maurice, Jonathan Ostry, and Mahvash Qureshi. 2017. "A Tie That Binds: Revisiting the Trilemma in Emerging Market Economies." IMF Working Paper WP/17/130. Washington, DC: International Monetary Fund. The one exception is that we rely on an alternative methodology of propensity score weighted regressions.

2. The dependent variable is the three-quarter moving average of quarterly net capital flows (in percent of GDP) in emerging market economies.

3. VXO is the precursor of the Chicago Board Options Exchange's Volatility Index or VIX.

4. Numbers in parentheses are clustered standard errors (by country).

${ }^{* * *},{ }^{* *},{ }^{*}$ indicate statistical significance at the $1 \%, 5 \%$, and $10 \%$ levels, respectively.

Source: Authors' calculations. 
16 | ADB Economics Working Paper Series No. 610

Table 1.8: Impact of VXO on Foreign Direct Investment, Portfolio, and Other Investment Liability Flows

\begin{tabular}{|c|c|c|c|c|c|c|c|c|c|c|c|c|}
\hline \multirow[b]{2}{*}{ Variables } & \multicolumn{4}{|c|}{ Real Credit Growth } & \multicolumn{4}{|c|}{ Real House Price Growth } & \multicolumn{4}{|c|}{ Change in LTD Ratio } \\
\hline & (1) & (2) & (3) & (4) & (5) & (6) & (7) & (8) & (9) & (10) & (11) & (12) \\
\hline \multirow{2}{*}{$\begin{array}{l}\text { Intermediate } \\
\text { regime }\end{array}$} & 0.848 & 1.155 & 1.209 & $4.780^{*}$ & 1.414 & 1.362 & 1.195 & -1.076 & -2.740 & -2.736 & -1.875 & 1.522 \\
\hline & (1.988) & $(2.104)$ & (1.581) & $(2.616)$ & $(1.659)$ & $(1.689)$ & $(1.365)$ & (1.983) & $(2.420)$ & $(2.424)$ & $(2.765)$ & $(2.961)$ \\
\hline \multirow[t]{2}{*}{ Fixed regime } & $6.912^{*}$ & $6.929^{*}$ & $6.683^{*}$ & $9.591^{* *}$ & 0.787 & 0.599 & 1.332 & -0.700 & 8.541 & $8.953^{*}$ & $7.125^{*}$ & $11.170^{*}$ \\
\hline & $(3.747)$ & $(3.745)$ & $(3.510)$ & $(4.451)$ & $(2.637)$ & $(2.567)$ & (3.233) & $(2.566)$ & (5.134) & $(5.151)$ & $(4.056)$ & $(5.800)$ \\
\hline \multirow[t]{2}{*}{$\log (V X O)$} & -0.110 & -0.061 & 0.441 & & $-0.947^{* *}$ & $-0.967^{* *}$ & $-1.092^{* * *}$ & & $-1.744^{* * *}$ & $-1.662^{* * *}$ & $-1.319^{* *}$ & \\
\hline & $(0.368)$ & $(0.360)$ & $(0.403)$ & & $(0.442)$ & $(0.458)$ & $(0.353)$ & & (0.595) & $(0.603)$ & $(0.645)$ & \\
\hline \multirow{2}{*}{$\begin{array}{l}\text { Intermediate } \times \\
\log (V X O)\end{array}$} & -0.118 & -0.214 & -0.464 & -1.020 & -0.385 & -0.374 & -0.305 & 0.156 & 0.902 & 0.879 & 0.511 & -0.195 \\
\hline & $(0.680)$ & $(0.726)$ & $(0.694)$ & $(0.848)$ & (0.523) & $(0.542)$ & $(0.456)$ & (0.618) & (0.735) & $(0.740)$ & (0.806) & $(0.851)$ \\
\hline \multirow{2}{*}{$\begin{array}{l}\text { Fixed } x \\
\log (V X O)\end{array}$} & $-2.360^{*}$ & $-2.340^{*}$ & $-2.394^{*}$ & $-2.819^{*}$ & 0.063 & 0.115 & 0.121 & 0.347 & -2.558 & -2.728 & -2.543 & $-3.047^{*}$ \\
\hline & (1.238) & $(1.237)$ & (1.218) & (1.418) & $(0.914)$ & $(0.885)$ & $(0.905)$ & (0.957) & (1.635) & (1.675) & (1.521) & (1.765) \\
\hline \multirow{2}{*}{$\begin{array}{l}\text { Lagged real } \\
\text { GDP growth }\end{array}$} & $0.101^{* *}$ & $0.098^{* *}$ & 0.069 & 0.051 & 0.018 & 0.019 & 0.026 & 0.065 & $0.241^{* * *}$ & $0.242^{* * *}$ & $0.214^{* * *}$ & $0.201^{* * *}$ \\
\hline & $(0.049)$ & $(0.048)$ & $(0.044)$ & $(0.045)$ & $(0.031)$ & $(0.031)$ & $(0.035)$ & $(0.040)$ & (0.075) & $(0.076)$ & $(0.072)$ & $(0.071)$ \\
\hline \multirow{2}{*}{$\begin{array}{l}\text { Lagged } \\
\text { institutional } \\
\text { quality }\end{array}$} & $7.112^{* * *}$ & $7.028^{* *}$ & $5.709^{* *}$ & 2.940 & 2.956 & 3.232 & 4.261 & 4.282 & $16.127^{* * *}$ & $15.019^{* *}$ & $13.568^{* *}$ & $11.954^{* *}$ \\
\hline & $(2.613)$ & $(2.606)$ & (2.331) & (3.939) & $(3.276)$ & (3.468) & (3.799) & (3.781) & (5.808) & (5.642) & (5.758) & $(4.453)$ \\
\hline \multirow{2}{*}{$\begin{array}{l}\text { Lagged } \\
\text { domestic } \\
\text { credit/GDP }\end{array}$} & 0.014 & 0.013 & 0.010 & $0.025^{*}$ & -0.012 & -0.012 & -0.012 & $-0.024^{*}$ & -0.009 & -0.007 & -0.010 & -0.007 \\
\hline & $(0.012)$ & $(0.012)$ & $(0.012)$ & $(0.013)$ & $(0.012)$ & $(0.012)$ & $(0.013)$ & $(0.012)$ & $(0.016)$ & $(0.016)$ & (0.019) & $(0.018)$ \\
\hline \multirow{2}{*}{$\begin{array}{l}\text { Real US T-bill } \\
\text { rate }\end{array}$} & & $0.096^{* * *}$ & & & & -0.048 & & & & -0.048 & & \\
\hline & & $(0.032)$ & & & & $(0.091)$ & & & & $(0.060)$ & & \\
\hline \multirow{2}{*}{$\begin{array}{l}\text { Intermediate } \times \\
\text { real US T-bill } \\
\text { rate }\end{array}$} & & 0.041 & & & & 0.017 & & & & 0.040 & & \\
\hline & & $(0.107)$ & & & & $(0.108)$ & & & & $(0.087)$ & & \\
\hline \multirow{2}{*}{$\begin{array}{l}\text { Fixed } \times \text { real US } \\
\text { T-bill rate }\end{array}$} & & -0.019 & & & & -0.064 & & & & $0.381^{*}$ & & \\
\hline & & $(0.079)$ & & & & $(0.152)$ & & & & $(0.201)$ & & \\
\hline \multirow{2}{*}{$\begin{array}{l}\text { Real shadow } \\
\text { federal funds }\end{array}$} & & & $0.302^{* * *}$ & & & & -0.098 & & & & $0.193^{* *}$ & \\
\hline & & & $(0.088)$ & & & & (0.154) & & & & $(0.092)$ & \\
\hline \multirow{2}{*}{$\begin{array}{l}\text { Intermediate } \times \\
\text { real shadow } \\
\text { rate }\end{array}$} & & & 0.293 & & & & 0.000 & & & & 0.102 & \\
\hline & & & $(0.241)$ & & & & $(0.175)$ & & & & $(0.129)$ & \\
\hline \multirow{2}{*}{$\begin{array}{l}\text { Fixed } \times \text { real } \\
\text { shadow rate }\end{array}$} & & & 0.196 & & & & -0.243 & & & & 0.510 & \\
\hline & & & $(0.165)$ & & & & $(0.291)$ & & & & $(0.335)$ & \\
\hline
\end{tabular}


Table 1.8 continued

\begin{tabular}{|c|c|c|c|c|c|c|c|c|c|c|c|c|}
\hline \multirow[b]{2}{*}{ Variables } & \multicolumn{4}{|c|}{ Real Credit Growth } & \multicolumn{4}{|c|}{ Real House Price Growth } & \multicolumn{4}{|c|}{ Change in LTD Ratio } \\
\hline & (1) & (2) & (3) & (4) & (5) & (6) & (7) & (8) & (9) & (10) & (11) & (12) \\
\hline \multirow[t]{2}{*}{ Linear trend } & 0.000 & 0.007 & $0.043^{* *}$ & & $0.018^{* *}$ & $0.014^{* *}$ & 0.004 & & $-0.027^{* *}$ & $-0.023^{*}$ & 0.005 & \\
\hline & $(0.006)$ & $(0.007)$ & $(0.017)$ & & $(0.007)$ & $(0.007)$ & $(0.010)$ & & $(0.012)$ & $(0.012)$ & $(0.015)$ & \\
\hline \multirow{2}{*}{$\begin{array}{l}\text { Global financial } \\
\text { crisis }\end{array}$} & 0.450 & 0.292 & 0.528 & & $-1.669^{* * *}$ & $-1.594^{* * *}$ & $-1.815^{* * *}$ & & -1.128 & -1.284 & -0.971 & \\
\hline & $(0.580)$ & $(0.535)$ & $(0.570)$ & & $(0.468)$ & $(0.463)$ & $(0.429)$ & & $(0.877)$ & $(0.891)$ & $(0.903)$ & \\
\hline $\begin{array}{l}\text { Quarter-year } \\
\text { effects }\end{array}$ & No & No & No & Yes & No & No & No & Yes & No & No & No & Yes \\
\hline Observations & 2,118 & 2,118 & 2,084 & 2,118 & 2,118 & 2,118 & 2,084 & 2,118 & 2,083 & 2,083 & 2,051 & 2,083 \\
\hline Adjusted $R^{2}$ & 0.384 & 0.387 & 0.405 & 0.425 & 0.190 & 0.191 & 0.203 & 0.230 & 0.340 & 0.346 & 0.360 & 0.435 \\
\hline $\begin{array}{l}\text { No. of } \\
\text { countries }\end{array}$ & 39 & 39 & 39 & 39 & 39 & 39 & 39 & 39 & 38 & 38 & 38 & 38 \\
\hline
\end{tabular}

$\mathrm{GDP}=$ gross domestic product, LTD = loan-to-deposit, US = United States.

Notes:

1. This is identical to Table 8 in Obstfeld, Maurice, Jonathan Ostry, and Mahvash Qureshi. 2017. "A Tie That Binds: Revisiting the Trilemma in Emerging Market Economies." IMF Working Paper WP/17/130. Washington, DC: International Monetary Fund. The one exception is that we rely on an alternative methodology of propensity score weighted regressions.

2. The dependent variable is the three-quarter moving average in emerging market economies' quarterly foreign direct investment liability flows (in percent of GDP) in columns (1)-(4), portfolio liability flows (in percent of GDP) in columns (5)-(8), and other investment liability flows (in percent of GDP) in columns (9)-(12).

3. VXO is the precursor of the Chicago Board Options Exchange's Volatility Index or VIX.

4. Numbers in parentheses are clustered standard errors (by country).

$* * * * *,{ }^{*}$ indicate statistical significance at the $1 \%, 5 \%$, and $10 \%$ levels, respectively.

Source: Authors' calculations. 
Table 1.9: Impact of VXO on Real Gross Domestic Product Growth

\begin{tabular}{|c|c|c|c|c|c|}
\hline \multirow[b]{2}{*}{ Variables } & $1986-2013$ & 1986-2013 & $1986-2013$ & $1986-2013$ & 1986-2013 \\
\hline & $(1)$ & (2) & (3) & (4) & (5) \\
\hline \multirow[t]{2}{*}{ Intermediate regime } & 0.574 & 0.474 & $1.068^{*}$ & $1.239^{* *}$ & $1.332^{* * *}$ \\
\hline & $(0.513)$ & $(0.513)$ & $(0.583)$ & $(0.556)$ & $(0.473)$ \\
\hline \multirow[t]{2}{*}{ Fixed regime } & $2.664^{* *}$ & $2.639^{* *}$ & $2.838^{* *}$ & $2.922^{* * *}$ & $2.654^{* *}$ \\
\hline & $(1.047)$ & $(1.040)$ & $(1.130)$ & $(0.997)$ & $(1.028)$ \\
\hline $\log (V X O)$ & $\begin{array}{l}-0.487^{* * *} \\
(0.122)\end{array}$ & $\begin{array}{l}-0.515^{* * *} \\
(0.122)\end{array}$ & $\begin{array}{l}-0.499^{* * *} \\
(0.141)\end{array}$ & & \\
\hline Intermediate $\times \log (\mathrm{VXO})$ & $\begin{array}{l}-0.196 \\
(0.171)\end{array}$ & $\begin{array}{l}-0.158 \\
(0.168)\end{array}$ & $\begin{array}{r}-0.286 \\
(0.177)\end{array}$ & $\begin{array}{l}-0.390^{* *} \\
(0.189)\end{array}$ & $\begin{array}{l}-0.356^{* *} \\
(0.165)\end{array}$ \\
\hline Fixed $\times \log (V X O)$ & $\begin{array}{l}-0.811^{* *} \\
(0.334)\end{array}$ & $\begin{array}{l}-0.807^{* *} \\
(0.332)\end{array}$ & $\begin{array}{l}-0.876^{* *} \\
(0.347)\end{array}$ & $\begin{array}{l}-0.882^{* *} \\
(0.333)\end{array}$ & $\begin{array}{c}-0.752^{* *} \\
(0.324)\end{array}$ \\
\hline Real US T-bill rate & & $\begin{array}{r}-0.004 \\
(0.019)\end{array}$ & & & \\
\hline Intermediate $\times$ real US T-bill rate & & $\begin{array}{l}-0.057^{* *} \\
(0.027)\end{array}$ & & & \\
\hline Fixed $\times$ real US T-bill rate & & $\begin{array}{l}-0.012 \\
(0.028)\end{array}$ & & & \\
\hline Real shadow federal funds & & & $\begin{array}{c}0.017 \\
(0.029)\end{array}$ & & \\
\hline Intermediate $\times$ real shadow rate & & & $\begin{array}{c}-0.079^{*} \\
(0.046)\end{array}$ & & \\
\hline Fixed $\times$ real shadow rate & & & $\begin{array}{l}-0.002 \\
(0.051)\end{array}$ & & \\
\hline Lagged net capital flows/GDP & $\begin{array}{c}-0.004 \\
(0.004)\end{array}$ & $\begin{array}{c}-0.004 \\
(0.004)\end{array}$ & $\begin{array}{l}-0.004 \\
(0.004)\end{array}$ & $\begin{array}{l}-0.008^{* *} \\
(0.004)\end{array}$ & $\begin{array}{c}-0.008^{* *} \\
(0.003)\end{array}$ \\
\hline Lagged institutional quality & $\begin{array}{c}-0.055 \\
(1.225)\end{array}$ & $\begin{array}{l}0.124 \\
(1.158)\end{array}$ & $\begin{array}{c}-0.077 \\
(1.085)\end{array}$ & $\begin{array}{l}0.638 \\
(1.324)\end{array}$ & $\begin{array}{c}2.475 \\
(1.494)\end{array}$ \\
\hline Lagged private credit/GDP & $\begin{array}{l}-0.014^{* * *} \\
(0.004)\end{array}$ & $\begin{array}{l}-0.013^{* * *} \\
(0.004)\end{array}$ & $\begin{array}{l}-0.013^{* * *} \\
(0.004)\end{array}$ & $\begin{array}{l}-0.012^{* *} \\
(0.005)\end{array}$ & $\begin{array}{c}-0.017^{* *} \\
(0.007)\end{array}$ \\
\hline Lagged real GDP per capita & $\begin{array}{l}-2.326^{* * *} \\
(0.711)\end{array}$ & $\begin{array}{l}-2.374^{* * *} \\
(0.687)\end{array}$ & $\begin{array}{l}-2.565^{* * *} \\
(0.742)\end{array}$ & $\begin{array}{l}-2.416^{* * *} \\
(0.676)\end{array}$ & $\begin{array}{l}-2.549^{* * *} \\
(0.771)\end{array}$ \\
\hline Linear trend & $\begin{array}{c}0.014^{* *} \\
(0.006)\end{array}$ & $\begin{array}{c}0.012^{* *} \\
(0.006)\end{array}$ & $\begin{array}{c}0.014^{* *} \\
(0.006)\end{array}$ & & \\
\hline Global financial crisis & $\begin{array}{l}-1.351^{* * *} \\
(0.332)\end{array}$ & $\begin{array}{l}-1.303^{* * *} \\
(0.332)\end{array}$ & $\begin{array}{l}-1.287^{* * *} \\
(0.327)\end{array}$ & & \\
\hline Quarter-year effects & No & No & No & Yes & Yes \\
\hline Observations & 2,103 & 2,103 & 2,071 & 2,103 & 1,635 \\
\hline Adjusted $R^{2}$ & 0.313 & 0.318 & 0.325 & 0.430 & 0.486 \\
\hline No. of countries & 38 & 38 & 38 & 38 & 38 \\
\hline
\end{tabular}

GDP = gross domestic product, US = United States.

Notes:

1. This is identical to Table 9 in Obstfeld, Maurice, Jonathan Ostry, and Mahvash Qureshi. 2017. "A Tie That Binds: Revisiting the Trilemma in Emerging Market Economies." IMF Working Paper WP/17/130. Washington, DC: International Monetary Fund. The one exception is that we rely on an alternative methodology of propensity score weighted regressions.

2. The dependent variable is the three-quarter moving average of quarterly (seasonally adjusted) real GDP growth rate (in percent) in emerging market economies.

3. VXO is the precursor of the Chicago Board Options Exchange's Volatility Index or VIX.

4. Numbers in parentheses are clustered standard errors (by country).

$* * *, * * *{ }^{*}$ indicate statistical significance at the $1 \%, 5 \%$, and $10 \%$ levels, respectively.

Source: Authors' calculations. 
We note the significance of fixed and intermediate exchange rate regimes in the nine sets of regressions. These regressions also produce results that closely resemble those of Obstfeld, Ostry, and Qureshi (2017). Exchange rate fixers tend to observe higher growth in credit, house prices, and GDP. They also tend to have higher loan-to-deposit ratios, net capital inflows, and liability and asset flows. Real stock returns, on the other hand, tend to be lower when countries adopted less flexible exchange rate regimes. Given the way exchange rate regimes are defined here, we can interpret those observations as indicating that more stable exchange rates are beneficial for promoting domestic credit conditions, asset price development, capital flows, and GDP growth in general. In other words, even de jure flexible regime adopters could also experience these described conditions so long as the volatility of their exchange rates remain limited. That said, while a stable exchange rate environment may promote the development of domestic financial conditions, its potential flexibility to move in responding to external shocks will help an economy to insulate the unwanted effects of these shocks.

To ensure the robustness of the findings, we extend the analysis by replacing the US-centric VXO with a more general representative of international shocks, the Londono-Wilson index of global volatility. Table 2 shows the relationship between these same dependent variables and the LondonoWilson index, which are very similar to those obtained when the VXO is used instead. To conserve space, we report only the coefficients of intermediate and fixed exchange rate regimes, and the LondonoWilson index and their interactions, which are all estimated using the same specifications in Table 1, Tables 1.1-1.7, and 1.9. We report results for the first five columns in each table, with row (1) corresponding to Table 1.1, row (2) to Table 1.2, and so on. ${ }^{11}$

Table 2: Impact of the Londono-Wilson Index on Various Variables in Emerging Market Economies

\begin{tabular}{|c|c|c|c|c|c|c|c|}
\hline & Dependent Variables & Explained Variables & (1) & $(2)$ & (3) & (4) & (5) \\
\hline \multirow{5}{*}[1]{} & \multirow{5}{*}{$\begin{array}{l}\text { Real domestic credit } \\
\text { growth }\end{array}$} & Intermediate & 0.3 & 2.4 & 2.5 & 2.5 & $3.8^{*}$ \\
\hline & & Fixed & 1.4 & $11.0^{* *}$ & $10.9^{* *}$ & $12.3^{* *}$ & $10.6^{* *}$ \\
\hline & & LW & $-1.7^{* * *}$ & -0.6 & -0.5 & 0.0 & \\
\hline & & Inter $\times$ LW & & -0.6 & -0.7 & -0.7 & -1.0 \\
\hline & & Fix $\times$ LW & & $-3.0^{* *}$ & $-3.0^{* *}$ & $-3.3^{* *}$ & $-2.8^{* *}$ \\
\hline \multirow[t]{5}{*}{ [2] } & \multirow[t]{5}{*}{ Real house price growth } & Intermediate & $1.6^{* * *}$ & -0.1 & -0.3 & 0.2 & 0.1 \\
\hline & & Fixed & 1.3 & $23.6^{* * *}$ & $23.7^{* * *}$ & $23.5^{* *}$ & $21.4^{* * *}$ \\
\hline & & LW & $-2.4^{* * *}$ & -0.3 & -0.3 & -0.2 & \\
\hline & & Inter $\times$ LW & & 0.6 & 0.7 & 0.5 & 0.7 \\
\hline & & Fix $\times$ LW & & $-6.8^{* * *}$ & $-6.8^{* * *}$ & $-6.8^{* *}$ & $-6.0^{* *}$ \\
\hline \multirow[t]{5}{*}{ [3] } & \multirow[t]{5}{*}{ Real stock returns } & Intermediate regime & 2.3 & -2.5 & -2.9 & -4.1 & 0.5 \\
\hline & & Fixed regime & 0.3 & 5.6 & 4.9 & 7.8 & 5.5 \\
\hline & & LW & $-8.3^{* * *}$ & $-8.3^{* * *}$ & $-9.6^{* * *}$ & $-11.3^{* * *}$ & \\
\hline & & Inter $\times$ LW & & 1.5 & 1.6 & 1.8 & 0.6 \\
\hline & & Fix $\times$ LW & & -1.7 & -1.6 & -2.1 & -1.9 \\
\hline \multirow[t]{5}{*}{ [4] } & \multirow{5}{*}{$\begin{array}{l}\text { Change in loan-to- } \\
\text { deposit ratio }\end{array}$} & Intermediate & -0.2 & 3.7 & 3.7 & 4.9 & 4.3 \\
\hline & & Fixed & 1.2 & $6.4^{*}$ & $6.4^{*}$ & $8.0^{* *}$ & $7.1^{* *}$ \\
\hline & & LW & -0.3 & 0.6 & 0.6 & $1.4^{* *}$ & \\
\hline & & Inter $\times \mathrm{LW}$ & & -1.2 & -1.2 & $-1.5^{* *}$ & $-1.4^{* *}$ \\
\hline & & Fix $\times$ LW & & $-1.6^{*}$ & $-1.6^{*}$ & $-2.0^{* *}$ & $-1.9^{* *}$ \\
\hline
\end{tabular}

continued on next page

${ }^{11}$ Additional results for other specifications available on request. 
Table 2 continued

\begin{tabular}{|c|c|c|c|c|c|c|c|}
\hline & Dependent Variables & Explained Variables & (1) & (2) & (3) & (4) & (5) \\
\hline \multirow[t]{5}{*}[5]{} & Net capital flows & Intermediate & $1.9^{* *}$ & $6.7^{*}$ & 6.3 & 4.7 & $7.4^{*}$ \\
\hline & & Fixed & 2.3 & $13.5^{* *}$ & $13.4^{* *}$ & $9.9^{*}$ & $13.7^{* *}$ \\
\hline & & & -0.7 & 0.8 & 0.9 & 1.4 & \\
\hline & & Inter $\times$ LW & & -1.5 & -1.4 & -1.2 & -1.6 \\
\hline & & Fix $\times$ LW & & $-3.5^{*}$ & $-3.4^{*}$ & $-2.7^{*}$ & $-3.4^{*}$ \\
\hline \multirow[t]{5}{*}{ [6] } & Liability flows & Intermediate & 1.2 & -1.6 & -2.3 & -6.0 & 0.4 \\
\hline & & Fixed & $3.5^{*}$ & 13.2 & 12.8 & 8.0 & $15.1^{*}$ \\
\hline & & LW & $-4.2^{* * *}$ & $-3.5^{* * *}$ & $-3.5^{* * *}$ & $-2.2^{* *}$ & \\
\hline & & Inter $\times$ LW & & 0.9 & 1.1 & 1.6 & 0.5 \\
\hline & & $\mathrm{Fix} \times \mathrm{LW}$ & & -3.0 & -2.8 & -2.0 & -3.3 \\
\hline \multirow[t]{5}{*}{ [7] } & Asset flows & Intermediate & 0.8 & $8.4^{* *}$ & $8.7^{* *}$ & $10.7^{* * *}$ & $7.0^{*}$ \\
\hline & & Fixed & -1.0 & 0.4 & 0.6 & 2.0 & -1.4 \\
\hline & & LW & $3.5^{* * *}$ & $4.3^{* * *}$ & $4.4^{* * *}$ & $3.6^{* * *}$ & \\
\hline & & Inter $\times$ LW & & $-2.4^{* *}$ & $-2.5^{* *}$ & $-2.8^{* * *}$ & $-2.1^{*}$ \\
\hline & & Fix $\times$ LW & & -0.4 & -0.6 & -0.7 & -0.1 \\
\hline \multirow[t]{5}{*}{ [8] } & Real GDP growth & Intermediate & 0.1 & $1.4^{* *}$ & $1.3^{* *}$ & $1.7^{* *}$ & $1.4^{* *}$ \\
\hline & & Fixed & 0.2 & $3.1^{* * *}$ & $3.1^{\text {t**}}$ & $2.8^{* *}$ & $3.1^{* * *}$ \\
\hline & & LW & $-0.6^{* * *}$ & -0.2 & $-0.3^{*}$ & -0.1 & \\
\hline & & Inter $\times$ LW & & $-0.4^{* *}$ & $-0.4^{*}$ & $-0.5^{* *}$ & $-0.4^{*}$ \\
\hline & & Fix $\times$ LW & & $-0.9^{* * *}$ & $-0.9^{* *}$ & $-0.8^{* *}$ & $-0.9^{* *}$ \\
\hline
\end{tabular}

GDP = gross domestic product.

Notes: Only the coefficients of intermediate and fixed exchange rate regimes, the Londono-Wilson (LW) index, and their interactions are reported, estimated using propensity score weighted regressions and the same dependent variables as in Table 1, Tables 1.1-1.7, and 1.9, which are listed in the first column.

***, **, indicate statistical significance at the 1\%, 5\%, and $10 \%$ levels, respectively, based on clustered standard errors (by country).

Source: Author's calculations.

In the same specification, an increase in the Londono-Wilson index has a significant negative effect on real domestic credit growth and real house price growth, especially for those adopting a fixed exchange rate regime. These hardline currency fixers also tend to observe the significant negative effect of the Londono-Wilson index on changes to their loan-to-deposit ratio or net capital flows. The index also negatively affects real stock returns and cross-border liability flows regardless of the adopted exchange rate regime. The effect on cross-border asset flows is positive and significant, as if the impact of increased volatility makes investment in the domestic economy less attractive for both foreign and domestic investors, resulting in both reduced liability inflows and increased resident-based asset outflows. This is less so, however, for countries with intermediate exchange rate regimes. The effect of the Londono-Wilson index on real GDP growth is negative, with larger negative effects observed in fixed regime economies. Of all the Londono-Wilson index results, only those on cross-border liability differ from what one finds when using the VXO as a measure of external volatility. This suggests that alternative volatility measures tend to yield similar results, although there are some differences.

In Tables 3-10, we use the eight fundamental variables making up the Londono-Wilson index, individually and in turn, as a global shock, and report the same five coefficients estimated by propensity score weighted regression methods. In Table 3, for example, we take US industrial production growth, the first component of the Londono-Wilson index, as the global shock. This can be thought of as capturing US-source nonfinancial shocks. This measure of external volatility has a larger positive impact - the domestic variable goes up when US industrial production goes up —on real domestic credit 


\section{Table 3: Impact of the United States Industrial Production Growth on Various Variables in Emerging Market Economies}

\begin{tabular}{|c|c|c|c|c|c|c|c|}
\hline & Dependent Variables & Explained Variables & (1) & (2) & (3) & (4) & (5) \\
\hline [1] & $\begin{array}{l}\text { Real domestic credit } \\
\text { growth }\end{array}$ & $\begin{array}{l}\text { Intermediate } \\
\text { Fixed } \\
\text { USIP } \\
\text { Inter } \times \text { USIP } \\
\text { Fix } \times \text { USIP }\end{array}$ & $\begin{array}{l}1.6^{*} \\
3.1^{* * *} \\
-2.7\end{array}$ & $\begin{array}{l}1.3^{*} \\
3.2^{* * *} \\
-8.5 \\
14.4^{* *} \\
-0.8\end{array}$ & $\begin{array}{l}1.3^{*} \\
3.1^{* * *} \\
-8.4 \\
14.1^{* *} \\
0.5\end{array}$ & $\begin{array}{l}0.5 \\
3.0^{* *} \\
-8.2 \\
10.8^{*} \\
-0.6\end{array}$ & $\begin{array}{l}0.7 \\
2.4^{* *} \\
18.7^{* * *} \\
3.9\end{array}$ \\
\hline$[2]$ & Real house price growth & $\begin{array}{l}\text { Intermediate } \\
\text { Fixed } \\
\text { USIP } \\
\text { Inter } \times \text { USIP } \\
\text { Fix } \times \text { USIP }\end{array}$ & $\begin{array}{l}1.3^{* * *} \\
0.8 \\
-8.0\end{array}$ & $\begin{array}{l}1.0^{* * *} \\
1.1 \\
-28.0^{* *} \\
12.0 \\
47.8^{* * *}\end{array}$ & $\begin{array}{c}1.0^{* * *} \\
1.2^{* *} \\
-28.0^{* *} \\
12.6 \\
48.1^{* * *}\end{array}$ & $\begin{array}{c}1.2^{* *} \\
1.1 \\
-28.0^{* *} \\
12.3 \\
46.9^{* * *}\end{array}$ & $\begin{array}{c}0.9 \\
1.0 \\
\\
6.3 \\
40.9^{* *}\end{array}$ \\
\hline$[3]$ & Real stock returns & $\begin{array}{l}\text { Intermediate } \\
\text { Fixed } \\
\text { USIP } \\
\text { Inter } \times \text { USIP } \\
\text { Fix } \times \text { USIP }\end{array}$ & $\begin{array}{c}0.9 \\
-1.3 \\
-19.7^{* *}\end{array}$ & $\begin{array}{r}1.0 \\
-1.7 \\
-18.3 \\
-17.2 \\
21.5\end{array}$ & $\begin{array}{r}0.7 \\
-2.2 \\
-21.2 \\
-15.0 \\
19.2\end{array}$ & $\begin{array}{r}0.0 \\
-0.8 \\
-12.3 \\
-23.7 \\
24.7\end{array}$ & $\begin{array}{c}2.5^{* * *} \\
-0.2 \\
1.6 \\
36.2^{*}\end{array}$ \\
\hline$[4]$ & $\begin{array}{l}\text { Change in loan-to- } \\
\text { deposit ratio }\end{array}$ & $\begin{array}{l}\text { Intermediate } \\
\text { Fixed } \\
\text { USIP } \\
\text { Inter } \times \text { USIP } \\
\text { Fix } \times \text { USIP }\end{array}$ & $\begin{array}{l}1.7^{* *} \\
4.4^{* *} \\
-3.9\end{array}$ & $\begin{array}{c}1.4^{*} \\
4.3^{* *} \\
-15.0^{*} \\
22.1^{* * *} \\
8.1\end{array}$ & $\begin{array}{c}1.4^{*} \\
4.3^{* *} \\
-14.9^{*} \\
22.0^{* * *} \\
8.0\end{array}$ & $\begin{array}{c}0.9 \\
3.6^{* * *} \\
-15.4^{*} \\
20.7^{* * *} \\
3.8\end{array}$ & $\begin{array}{l}0.8 \\
2.7^{* *} \\
20.2^{* * *} \\
6.2\end{array}$ \\
\hline$[5]$ & Net capital flows & $\begin{array}{l}\text { Intermediate } \\
\text { Fixed } \\
\text { USIP } \\
\text { Inter } \times \text { USIP } \\
\text { Fix } \times \text { USIP }\end{array}$ & $\begin{array}{l}1.9^{* * *} \\
1.3 \\
-5.0\end{array}$ & $\begin{array}{l}1.8^{* * *} \\
1.4 \\
-5.6 \\
6.3 \\
-6.0\end{array}$ & $\begin{array}{l}1.7^{* * *} \\
1.4 \\
-5.6 \\
6.5 \\
-5.7\end{array}$ & $\begin{array}{r}1.2 \\
0.9 \\
-6.5 \\
4.8 \\
-9.1\end{array}$ & $\begin{array}{l}2.1^{* *} \\
2.2 \\
\\
9.9 \\
-2.5\end{array}$ \\
\hline [6] & Liability flows & $\begin{array}{l}\text { Intermediate } \\
\text { Fixed } \\
\text { USIP } \\
\text { Inter } \times \text { USIP } \\
\text { Fix } \times \text { USIP }\end{array}$ & $\begin{array}{l}0.9 \\
2.0 \\
1.9\end{array}$ & $\begin{array}{r}1.2^{*} \\
2.5 \\
15.3 \\
-16.6 \\
-21.2\end{array}$ & $\begin{array}{r}1.1^{*} \\
2.4 \\
15.0 \\
-16.1 \\
-20.6\end{array}$ & $\begin{array}{r}-0.2 \\
1.6 \\
12.0 \\
-19.9 \\
-26.3\end{array}$ & $\begin{array}{l}2.2^{* *} \\
4.1^{\text {}} \\
-11.9 \\
-18.9\end{array}$ \\
\hline$[7]$ & Asset flows & $\begin{array}{l}\text { Intermediate } \\
\text { Fixed } \\
\text { USIP } \\
\text { Inter } \times \text { USIP } \\
\text { Fix } \times \text { USIP }\end{array}$ & $\begin{array}{c}1.0 \\
-0.7 \\
-7.2^{* *}\end{array}$ & $\begin{array}{l}0.6 \\
-1.0 \\
-21.0^{* * *} \\
22.8^{* * *} \\
14.7\end{array}$ & $\begin{array}{c}0.7 \\
-0.9 \\
-20.7^{* * *} \\
22.5^{\text {*}} \\
14.3\end{array}$ & 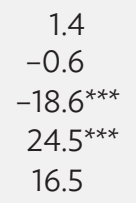 & $\begin{array}{l}-0.2 \\
-1.8^{* *} \\
22.0^{* *} \\
15.7\end{array}$ \\
\hline [8] & Real GDP growth & $\begin{array}{l}\text { Intermediate } \\
\text { Fixed } \\
\text { USIP } \\
\text { Inter } \times \text { USIP } \\
\text { Fix } \times \text { USIP }\end{array}$ & 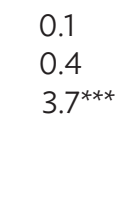 & $\begin{array}{c}0.1 \\
0.3 \\
3.1^{*} \\
-1.7 \\
4.4\end{array}$ & $\begin{array}{c}0.1 \\
0.2 \\
3.1^{*} \\
-1.7 \\
4.3\end{array}$ & $\begin{array}{l}0.1 \\
0.2 \\
3.0^{* *} \\
-1.8 \\
4.3\end{array}$ & $\begin{array}{r}0.1 \\
0.2 \\
\\
-3.1 \\
3.9\end{array}$ \\
\hline
\end{tabular}

GDP = gross domestic product, USIP = US industrial production.

Notes: Only the coefficients of intermediate and fixed exchange rate regimes, USIP growth, and their interactions, are reported, estimated using propensity score weighted regressions and the same dependent variables as in Table 1, Tables 1.1-1.7, and 1.9, which are listed in the first column.

***, **, ${ }^{*}$ indicate statistical significance at the 1\%, 5\%, and 10\% levels, respectively, based on clustered standard errors (by country).

Source: Author's calculations. 
growth, real house price growth, loan-to-deposit ratio growth, and cross-border asset flows in countries with less flexible exchange rate regimes. But it is intermediate rather than fixed rate regimes that appear to matter most for domestic credit growth and loan-to-deposit rate growth.

An alternative measure of US-source nonfinancial shocks, which is also an element of the Londono-Wilson index, is the probability of a US recession within the coming quarter. In Table 4, this has a larger negative effect on real domestic credit growth, real house price growth, loan-to-deposit ratio growth, and asset flows (the same variables as before) in countries with less flexible exchange rate regimes. But again, it appears to be intermediate regimes that matter most for the change in loan-todeposit ratios. Both intermediate and pegged rate regimes appear to amplify the impact of US recessions on real domestic credit growth, while intermediate regimes matter most for cross-border asset flows.

Table 5 shows the results for the last of our three US-source nonfinancial sources of volatility, the US Economic Policy Uncertainty Index. This variable has a heightened impact on real domestic credit growth, real house price growth, loan-to-deposit ratio growth, and net capital flows under less flexible exchange rate regimes. But while fixed rate regimes matter the most for real domestic credit growth, both regimes appear to accentuate the impact of US policy uncertainty on the growth of the loan-to-deposit ratio and net capital flows.

The next set of tables considers US financial variables as measures of external volatility. Table 6 indicates that the federal funds rate has a heightened impact on real domestic credit growth, the change in the loan-to-deposit ratio, and net capital flows under less flexible exchange rates. But domestic credit growth and net capital flows are affected most strongly by intermediate rates, while the change in the loan-to-deposit ratio is similarly affected under the two relatively inflexible exchange rate regimes.

Table 7 considers changes in the total assets of the Federal Reserve as a share of US GDP as a measure of unconventional and conventional monetary policy. This volatility measure has an outsized impact on real domestic credit growth, real house price growth, net capital flows, and real GDP growth in emerging markets with less flexible exchange rates. But that heightened impact is associated with fixed rates in the cases of real house price growth and real GDP growth, but with fixed and intermediate rates in the cases of net capital flows and asset flows.

Tables 8 and 9 consider a pair of non-US source measures of volatility: non-US industrial production growth and global economic policy uncertainty. In Table 8, less flexible exchange rates accentuate the impact of non-US industrial production growth on real house price growth, the change in the loan-to-deposit ratio, liability capital flows, asset flows, and real GDP growth in emerging markets. The impact on real house price growth and real GDP growth is accentuated mainly by fixed rates, but the change in the loan-to-deposit ratio and asset flows is accentuated mainly by intermediate rates. Notably, the results in Table 8 for the impact of non-US industrial production on GDP growth in emerging markets is the first time we identified an impact on a real (as opposed to a purely financial) dependent variable.

Table 9 considers global economic policy uncertainty. Less flexible exchange rate regimes accentuate the impact of this volatility measure on real domestic credit growth, real house price growth, changes in the loan-to-deposit ratio, net capital flows, and liability flows in emerging markets. But while fixed rate regimes mainly matter for real domestic credit growth, real house price growth, the change in the loan-to-deposit ratio, and liability capital flows, both fixed and intermediate regimes matter for net capital flows. 
Table 4: Impact of the Expected Probability of a United States Recession on Various Variables in Emerging Market Economies

\begin{tabular}{|c|c|c|c|c|c|c|c|}
\hline & Dependent Variables & Explained Variables & (1) & (2) & (3) & (4) & (5) \\
\hline$[1]$ & $\begin{array}{l}\text { Real domestic credit } \\
\text { growth }\end{array}$ & $\begin{array}{l}\text { Intermediate } \\
\text { Fixed } \\
\text { EPUS } \\
\text { Inter } \times \text { EPUSR } \\
\text { Fix } \times \text { EPUSR }\end{array}$ & $\begin{array}{c}1.6^{*} \\
3.1^{* *} \\
-0.3\end{array}$ & $\begin{array}{l}3.4^{* *} \\
5.9^{* *} \\
0.2 \\
-0.7^{*} \\
-1.1\end{array}$ & $\begin{array}{l}3.5^{* *} \\
6.0^{* *} \\
0.2 \\
-0.7^{*} \\
-1.1^{*}\end{array}$ & $\begin{array}{l}1.9 \\
6.4^{* * *} \\
0.2 \\
-0.6 \\
-1.3^{* *}\end{array}$ & $\begin{array}{l}2.9^{* *} \\
5.1^{* *} \\
-0.7 \\
-1.0\end{array}$ \\
\hline$[2]$ & Real house price growth & $\begin{array}{l}\text { Intermediate } \\
\text { Fixed } \\
\text { EPUSR } \\
\text { Inter } \times \text { EPUSR } \\
\text { Fix } \times \text { EPUSR }\end{array}$ & $\begin{array}{l}1.4^{* * *} \\
1.1 \\
-0.3\end{array}$ & $\begin{array}{l}1.9 \\
10.3^{* *} \\
0.9 \\
-0.2 \\
-3.0^{* *}\end{array}$ & $\begin{array}{l}2.1 \\
10.4^{* *} \\
0.9 \\
-0.2 \\
-3.1^{*}\end{array}$ & $\begin{array}{c}2.3 \\
10.0^{*} \\
0.9 \\
-0.2 \\
-2.9^{*}\end{array}$ & $\begin{array}{c}-0.6 \\
7.7^{*} \\
0.6 \\
-2.3\end{array}$ \\
\hline [3] & Real stock returns & $\begin{array}{l}\text { Intermediate } \\
\text { Fixed } \\
\text { EPUSR } \\
\text { Inter } \times \text { EPUSR } \\
\text { Fix } \times \text { EPUSR }\end{array}$ & $\begin{array}{l}1.4 \\
-0.6 \\
-3.9^{* * *}\end{array}$ & $\begin{array}{l}-1.7 \\
5.5 \\
-3.7^{* * *} \\
1.1 \\
-2.2\end{array}$ & $\begin{array}{l}-1.9 \\
4.2 \\
-3.4^{* * *} \\
1.1 \\
-2.0\end{array}$ & $\begin{array}{l}-4.0 \\
6.3 \\
-4.0^{* * *} \\
1.5 \\
-2.3\end{array}$ & $\begin{array}{r}-0.3 \\
4.7 \\
\\
1.1 \\
-1.7\end{array}$ \\
\hline [4] & $\begin{array}{l}\text { Change in loan-to- } \\
\text { deposit ratio }\end{array}$ & $\begin{array}{l}\text { Intermediate } \\
\text { Fixed } \\
\text { EPUSR } \\
\text { Inter } \times \text { EPUSR } \\
\text { Fix } \times \text { EPUSR }\end{array}$ & $\begin{array}{l}1.6^{* *} \\
4.2^{* *} \\
0.4\end{array}$ & $\begin{array}{l}4.8^{* * *} \\
6.2^{* *} \\
1.0^{* *} \\
-1.2^{* * *} \\
-0.8\end{array}$ & $\begin{array}{l}4.9^{* * *} \\
6.3^{* *} \\
1.0^{* *} \\
-1.3^{* * *} \\
-0.8\end{array}$ & $\begin{array}{l}3.9^{* * *} \\
5.4^{* * *} \\
1.0^{* *} \\
-1.2^{* * *} \\
-0.7\end{array}$ & $\begin{array}{l}3.9^{* * *} \\
4.0^{*} \\
-1.1^{1 * *} \\
-0.5\end{array}$ \\
\hline [5] & Net capital flows & $\begin{array}{l}\text { Intermediate } \\
\text { Fixed } \\
\text { EPUSR } \\
\text { Inter } \times \text { EPUSR } \\
\text { Fix } \times \text { EPUSR }\end{array}$ & $\begin{array}{l}1.8^{* * *} \\
1.1 \\
0.5^{*}\end{array}$ & $\begin{array}{l}3.4^{* *} \\
0.0 \\
0.6 \\
-0.6 \\
0.4\end{array}$ & $\begin{array}{l}3.5^{* *} \\
0.2 \\
0.6 \\
-0.7 \\
0.4\end{array}$ & $\begin{array}{r}2.7 \\
-0.6 \\
0.5 \\
-0.6 \\
0.5\end{array}$ & $\begin{array}{r}3.8^{*} \\
0.6 \\
-0.6 \\
0.6\end{array}$ \\
\hline [6] & Liability flows & $\begin{array}{l}\text { Intermediate } \\
\text { Fixed } \\
\text { EPUSR } \\
\text { Inter } \times \text { EPUSR } \\
\text { Fix } \times \text { EPUSR }\end{array}$ & $\begin{array}{r}0.9 \\
2.0 \\
-0.3\end{array}$ & $\begin{array}{r}-0.1 \\
0.2 \\
-0.6 \\
0.4 \\
0.7\end{array}$ & $\begin{array}{r}0.1 \\
0.5 \\
-0.6 \\
0.3 \\
0.6\end{array}$ & $\begin{array}{r}-1.8 \\
-0.9 \\
-0.7 \\
0.6 \\
0.9\end{array}$ & $\begin{array}{l}0.8 \\
1.2 \\
\\
0.5 \\
1.0\end{array}$ \\
\hline [7] & Asset flows & $\begin{array}{l}\text { Intermediate } \\
\text { Fixed } \\
\text { EPUSR } \\
\text { Inter } \times \text { EPUSR } \\
\text { Fix } \times \text { EPUSR }\end{array}$ & $\begin{array}{c}0.9 \\
-0.8 \\
0.8^{* * *}\end{array}$ & $\begin{array}{l}3.5^{* *} \\
-0.2 \\
1 . .^{\text {***}} \\
-1.0^{* *} \\
-0.2\end{array}$ & $\begin{array}{l}3.4^{* *} \\
-0.3 \\
1.1^{* * *} \\
-0.9^{*} \\
-0.2\end{array}$ & $\begin{array}{l}4.5^{*} \\
0.3 \\
1.2^{* * *} \\
-1.2^{* *} \\
-0.4\end{array}$ & $\begin{array}{l}3.1^{*} \\
-0.5 \\
-1.1^{* *} \\
-0.4\end{array}$ \\
\hline [8] & Real GDP growth & $\begin{array}{l}\text { Intermediate } \\
\text { Fixed } \\
\text { EPUSR } \\
\text { Inter } \times \text { EPUSR } \\
\text { Fix } \times \text { EPUSR }\end{array}$ & $\begin{array}{c}0.1 \\
0.4^{*} \\
-0.3^{* * *}\end{array}$ & $\begin{array}{l}-0.1 \\
1.0^{*} \\
-0.2^{* *} \\
0.1 \\
-0.2\end{array}$ & $\begin{array}{l}-0.1 \\
1.0^{*} \\
-0.2^{* *} \\
0.1 \\
-0.2\end{array}$ & $\begin{array}{l}-0.2 \\
0.9 \\
-0.2^{* * *} \\
0.1 \\
-0.2\end{array}$ & $\begin{array}{r}-0.5 \\
0.8 \\
0.2^{*} \\
-0.2\end{array}$ \\
\hline
\end{tabular}

EPUSR = expected probability of a United States recession, GDP = gross domestic product.

Notes: Only the coefficients of intermediate and fixed exchange rate regimes, expected probability of a US recession, and their interactions are reported, estimated using propensity score weighted regressions and the same dependent variables as in Table 1, Tables 1.1-1.7, and 1.9, which are listed in the first column.

${ }_{* * *}, * *,{ }^{*}$ indicate statistical significance at the $1 \%, 5 \%$, and $10 \%$ levels, respectively, based on clustered standard errors (by country).

Source: Author's calculations. 
Table 5: Impact of the United States Economic Policy Uncertainty Index on Various Variables in Emerging Market Economies

\begin{tabular}{|c|c|c|c|c|c|c|c|}
\hline & Dependent Variables & Explained Variables & (1) & (2) & (3) & (4) & (5) \\
\hline [1] & $\begin{array}{l}\text { Real domestic credit } \\
\text { growth }\end{array}$ & $\begin{array}{l}\text { Intermediate } \\
\text { Fixed } \\
\text { USEPU } \\
\text { Inter } \times \text { USEPU } \\
\text { Fix } \times \text { USEPU }\end{array}$ & $\begin{array}{c}1.6^{* *} \\
3.0^{* * *} \\
-1.0^{* *}\end{array}$ & $\begin{array}{l}6.8^{* *} \\
12.3^{* *} \\
-0.1 \\
-1.1^{*} \\
-2.0^{*}\end{array}$ & $\begin{array}{l}5.9^{*} \\
14.2^{* * *} \\
-0.0 \\
-0.9 \\
-2.4^{* *}\end{array}$ & $\begin{array}{l}-0.8 \\
16.7^{* * *} \\
-0.3 \\
0.2 \\
-2.8^{* *}\end{array}$ & $\begin{array}{l}5.0 \\
11.1^{* *} \\
-0.9 \\
-1.9^{*}\end{array}$ \\
\hline$[2]$ & Real house price growth & $\begin{array}{l}\text { Intermediate } \\
\text { Fixed } \\
\text { USEPU } \\
\text { Inter } \times \text { USEPU } \\
\text { Fix } \times \text { USEPU }\end{array}$ & $\begin{array}{l}1.3^{* * *} \\
1.0 \\
-1.3^{* *}\end{array}$ & $\begin{array}{l}-0.9 \\
12.4^{* *} \\
-0.7^{*} \\
0.5 \\
-2.4^{* *}\end{array}$ & $\begin{array}{l}-0.9 \\
13.1^{* *} \\
-0.6^{*} \\
0.5 \\
-2.5^{* *}\end{array}$ & $\begin{array}{c}2.3 \\
16.5^{*} \\
-0.4 \\
-0.1 \\
-3.1^{*}\end{array}$ & $\begin{array}{r}-6.7 \\
6.4 \\
\\
1.7 \\
-1.2\end{array}$ \\
\hline [3] & Real stock returns & $\begin{array}{l}\text { Intermediate } \\
\text { Fixed } \\
\text { USEPU } \\
\text { Inter } \times \text { USEPU } \\
\text { Fix } \times \text { USEPU }\end{array}$ & $\begin{array}{l}2.5^{* * *} \\
-0.1 \\
-5.8^{* * *}\end{array}$ & $\begin{array}{l}-0.4 \\
-0.9 \\
-6.0^{* * *} \\
0.6 \\
0.2\end{array}$ & $\begin{array}{l}1.9 \\
1.6 \\
-6.6^{* * *} \\
0.1 \\
-0.5\end{array}$ & $\begin{array}{c}-8.2 \\
7.8 \\
-10.5^{\text {*** }} \\
2.0 \\
-1.3\end{array}$ & $\begin{array}{r}0.4 \\
-5.8 \\
\\
0.5 \\
1.3\end{array}$ \\
\hline$[4]$ & $\begin{array}{l}\text { Change in loan-to- } \\
\text { deposit ratio }\end{array}$ & $\begin{array}{l}\text { Intermediate } \\
\text { Fixed } \\
\text { USEPU } \\
\text { Inter } \times \text { USEPU } \\
\text { Fix } \times \text { USEPU }\end{array}$ & $\begin{array}{l}1.5^{* *} \\
4.2^{* *} \\
-0.1\end{array}$ & $\begin{array}{l}7.8^{* * *} \\
11.3^{* * *} \\
0.7^{*} \\
-1.4^{* *} \\
-1.5^{* *}\end{array}$ & $\begin{array}{l}7.4^{* * *} \\
11.5^{* * *} \\
0.7 \\
-1.3^{* *} \\
-1.6^{* *}\end{array}$ & $\begin{array}{c}4.1 \\
8.2 \\
0.9^{*} \\
-0.7 \\
-1.0\end{array}$ & $\begin{array}{l}6.0^{*} \\
8.7^{* *} \\
-1.1^{*} \\
-1.3^{*}\end{array}$ \\
\hline [5] & Net capital flows & $\begin{array}{l}\text { Intermediate } \\
\text { Fixed } \\
\text { USEPU } \\
\text { Inter } \times \text { USEPU } \\
\text { Fix } \times \text { USEPU }\end{array}$ & $\begin{array}{l}2.0^{* * *} \\
1.3 \\
-1.4^{*}\end{array}$ & $\begin{array}{l}10.6^{* *} \\
16.5 \\
0.1 \\
-1.9^{*} \\
-3.3\end{array}$ & $\begin{array}{l}10.4^{* *} \\
16.6 \\
0.2 \\
-1.9^{*} \\
-3.3\end{array}$ & $\begin{array}{l}7.6 \\
21.0^{* * *} \\
0.6 \\
-1.3 \\
-4.1^{* *}\end{array}$ & $\begin{array}{r}8.0 \\
16.0 \\
-1.3 \\
-3.1\end{array}$ \\
\hline$[6]$ & Liability flows & $\begin{array}{l}\text { Intermediate } \\
\text { Fixed } \\
\text { USEPU } \\
\text { Inter } \times \text { USEPU } \\
\text { Fix } \times \text { USEPU }\end{array}$ & $\begin{array}{l}1.4^{* *} \\
2.5 \\
-4.0^{* * *}\end{array}$ & $\begin{array}{l}9.5 \\
19.0 \\
-2.4^{* * *} \\
-1.8 \\
-3.6\end{array}$ & $\begin{array}{l}7.9 \\
18.7^{*} \\
-2.4^{* * *} \\
-1.5 \\
-3.5\end{array}$ & $\begin{array}{l}2.0 \\
22.6^{* *} \\
-1.6^{*} \\
-0.4 \\
-4.2^{* *}\end{array}$ & $\begin{array}{r}7.9 \\
19.0 \\
\\
-1.4 \\
-3.4\end{array}$ \\
\hline [7] & Asset flows & $\begin{array}{l}\text { Intermediate } \\
\text { Fixed } \\
\text { USEPU } \\
\text { Inter } \times \text { USEPU } \\
\text { Fix } \times \text { USEPU }\end{array}$ & $\begin{array}{l}0.5 \\
-1.1 \\
2.5^{\text {*** }}\end{array}$ & $\begin{array}{l}1.3 \\
-2.4 \\
2.5^{* * *} \\
-0.2 \\
0.3\end{array}$ & $\begin{array}{l}2.7 \\
-2.0 \\
2.6^{* * *} \\
-0.4 \\
0.2\end{array}$ & $\begin{array}{l}5.8 \\
-1.3 \\
2.1^{* * *} \\
-1.0 \\
0.1\end{array}$ & $\begin{array}{r}0.3 \\
-2.9 \\
-0.0 \\
0.3\end{array}$ \\
\hline [8] & Real GDP growth & $\begin{array}{l}\text { Intermediate } \\
\text { Fixed } \\
\text { USEPU } \\
\text { Inter } \times \text { USEPU } \\
\text { Fix } \times \text { USEPU }\end{array}$ & $\begin{array}{c}0.2^{*} \\
0.5^{* *} \\
-0.4^{* * *}\end{array}$ & $\begin{array}{l}-0.5 \\
0.9 \\
-0.5^{* * *} \\
0.2 \\
-0.1\end{array}$ & $\begin{array}{l}-0.3 \\
1.0 \\
-0.5^{* * *} \\
0.1 \\
-0.1\end{array}$ & $\begin{array}{l}-0.9 \\
0.5 \\
-0.6^{* * *} \\
0.2 \\
-0.0\end{array}$ & $\begin{array}{r}-0.9 \\
0.8 \\
\\
0.2 \\
-0.1\end{array}$ \\
\hline
\end{tabular}

GDP = gross domestic product, USEPU = United States Economic Policy Uncertainty Index.

Notes: Only the coefficients of intermediate and fixed exchange rate regimes, the US Economic Policy Uncertainty Index, and their interactions are reported, estimated using propensity score weighted regressions and the same dependent variables as in Table 1, Tables 1.1-1.7, and 1.9, which are listed in the first column.

***, ${ }^{* *},{ }^{*}$ indicate statistical significance at the $1 \%, 5 \%$, and $10 \%$ levels, respectively, based on clustered standard errors (by country).

Source: Author's calculations. 
Table 6: Impact of the Federal Funds Rate on Various Variables in Emerging Market Economies

\begin{tabular}{|c|c|c|c|c|c|c|c|}
\hline & Dependent Variables & Explained Variables & (1) & (2) & (3) & (4) & (5) \\
\hline [1] & $\begin{array}{l}\text { Real domestic credit } \\
\text { growth }\end{array}$ & $\begin{array}{l}\text { Intermediate } \\
\text { Fixed } \\
\text { FFR } \\
\text { Inter } \times \text { FFR } \\
\text { Fix } \times \text { FFR }\end{array}$ & $\begin{array}{l}1.6^{* *} \\
3.1^{* * *} \\
0.2^{*}\end{array}$ & $\begin{array}{l}0.7 \\
2.7^{* *} \\
0.0 \\
0.4^{* * *} \\
0.2\end{array}$ & $\begin{array}{l}0.4 \\
2.0^{*} \\
-0.1 \\
0.5^{* * *} \\
0.4^{* *}\end{array}$ & & $\begin{array}{l}0.4 \\
2.0^{*} \\
0.2 \\
0.2\end{array}$ \\
\hline [2] & Real house price growth & $\begin{array}{l}\text { Intermediate } \\
\text { Fixed } \\
\text { FFR } \\
\text { Inter } \times \text { FFR } \\
\text { Fix } \times \text { FFR }\end{array}$ & $\begin{array}{l}1.3^{* * *} \\
1.0 \\
0.1\end{array}$ & $\begin{array}{l}1.8^{* * *} \\
1.3 \\
0.2 \\
-0.2 \\
-0.1\end{array}$ & $\begin{array}{l}1.9^{* * *} \\
1.2 \\
0.2 \\
-0.3 \\
-0.1\end{array}$ & & $\begin{array}{l}1.7^{* * *} \\
1.2 \\
-0.3 \\
-0.2\end{array}$ \\
\hline [3] & Real stock returns & $\begin{array}{l}\text { Intermediate } \\
\text { Fixed } \\
\text { FFR } \\
\text { Inter } \times \text { FFR } \\
\text { Fix } \times \text { FFR }\end{array}$ & $\begin{array}{c}0.1 \\
-2.1 \\
-0.5^{* *}\end{array}$ & $\begin{array}{l}-0.2 \\
-1.3 \\
-0.5^{* * *} \\
0.2 \\
-0.2\end{array}$ & $\begin{array}{r}0.4 \\
-1.3 \\
0.2 \\
0.1 \\
-0.2\end{array}$ & & $\begin{array}{l}3.5^{* *} \\
2.4 \\
-0.0 \\
-0.7^{* *}\end{array}$ \\
\hline [4] & $\begin{array}{l}\text { Change in loan-to- } \\
\text { deposit ratio }\end{array}$ & $\begin{array}{l}\text { Intermediate } \\
\text { Fixed } \\
\text { FFR } \\
\text { Inter } \times \text { FFR } \\
\text { Fix } \times \text { FFR }\end{array}$ & $\begin{array}{l}1.8^{* *} \\
4.5^{* * *} \\
0.2^{* *}\end{array}$ & $\begin{array}{l}1.2^{*} \\
3.7^{* * *} \\
0.1 \\
0.2^{*} \\
0.3\end{array}$ & $\begin{array}{l}1.1 \\
3.4^{* * *} \\
0.1 \\
0.3^{*} \\
0.4^{*}\end{array}$ & & $\begin{array}{l}1.0 \\
2.6^{* *} \\
0.1 \\
0.1\end{array}$ \\
\hline [5] & Net capital flows & $\begin{array}{l}\text { Intermediate } \\
\text { Fixed } \\
\text { FFR } \\
\text { Inter } \times \text { FFR } \\
\text { Fix } \times \text { FFR }\end{array}$ & $\begin{array}{l}2.0^{* * *} \\
1.5 \\
0.4\end{array}$ & $\begin{array}{l}1.0 \\
0.5 \\
0.2 \\
0.3^{*} \\
0.4\end{array}$ & $\begin{array}{l}0.8 \\
0.5 \\
0.1 \\
0.4^{*} \\
0.4\end{array}$ & & $\begin{array}{l}1.5 \\
1.2 \\
\\
0.1 \\
0.3\end{array}$ \\
\hline [6] & Liability flows & $\begin{array}{l}\text { Intermediate } \\
\text { Fixed } \\
\text { FFR } \\
\text { Inter } \times \text { FFR } \\
\text { Fix } \times \text { FFR }\end{array}$ & $\begin{array}{l}1.4^{*} \\
2.7 \\
0.9^{\text {*** }}\end{array}$ & $\begin{array}{l}-0.2 \\
1.3 \\
0.6^{* * *} \\
0.6^{*} \\
0.5\end{array}$ & $\begin{array}{l}0.1 \\
1.6 \\
0.8^{* * *} \\
0.4 \\
0.4\end{array}$ & & $\begin{array}{l}0.8 \\
2.6 \\
\\
0.3 \\
0.4\end{array}$ \\
\hline [7] & Asset flows & $\begin{array}{l}\text { Intermediate } \\
\text { Fixed } \\
\text { FFR } \\
\text { Inter } \times \text { FFR } \\
\text { Fix } \times \text { FFR }\end{array}$ & $\begin{array}{c}0.6 \\
-1.1 \\
-0.5^{\text {*** }}\end{array}$ & $\begin{array}{l}1.2 \\
-0.7 \\
-0.4^{* * *} \\
-0.2 \\
-0.2\end{array}$ & $\begin{array}{l}0.7 \\
-1.0 \\
-0.7^{* * *} \\
-0.0 \\
-0.0\end{array}$ & & $\begin{array}{r}0.6 \\
-1.3 \\
\\
-0.2 \\
-0.1\end{array}$ \\
\hline [8] & Real GDP growth & $\begin{array}{l}\text { Intermediate } \\
\text { Fixed } \\
\text { FFR } \\
\text { Inter } \times \text { FFR } \\
\text { Fix } \times \text { FFR }\end{array}$ & $\begin{array}{l}0.1 \\
0.4^{*} \\
0.0\end{array}$ & $\begin{array}{r}0.1 \\
0.3 \\
0.0 \\
-0.0 \\
0.0\end{array}$ & $\begin{array}{l}0.1 \\
0.3 \\
0.1 \\
0.0 \\
0.0\end{array}$ & & $\begin{array}{l}0.2 \\
0.3 \\
-0.1 \\
-0.0\end{array}$ \\
\hline
\end{tabular}

$\mathrm{FFR}$ = federal funds rate, GDP = gross domestic product.

Notes:

1 Only the coefficients of intermediate and fixed exchange rate regimes, the federal funds rate, and their interactions are reported, estimated using propensity score weighted regressions and the same dependent variables as in Table 1, Tables 1.1-1.7, and 1.9, which are listed in the first column.

2. The results in column (4) are not reported because the real shadow funds rate is also included as a regressor.

${ }^{* * *},{ }^{* *},{ }^{*}$ indicate statistical significance at the $1 \%, 5 \%$, and $10 \%$ levels, respectively, based on clustered standard errors (by country).

Source: Author's calculations. 
Table 7: Impact of the Change in Total Assets of the Federal Reserve on Various Variables in Emerging Market Economies

\begin{tabular}{|c|c|c|c|c|c|c|c|}
\hline & Dependent Variables & Explained Variables & (1) & (2) & (3) & (4) & (5) \\
\hline [1] & $\begin{array}{l}\text { Real domestic credit } \\
\text { growth }\end{array}$ & $\begin{array}{l}\text { Intermediate } \\
\text { Fixed } \\
\Delta \text { FedA } / Y \\
\text { Inter } \times \Delta \text { Fed } A / Y \\
\text { Fix } \times \Delta \text { FedA } / Y\end{array}$ & $\begin{array}{l}1.0 \\
2.4^{* *} \\
-2.6\end{array}$ & $\begin{array}{c}1.0 \\
2.7^{* *} \\
34.7^{* * *} \\
-34.7^{* *} \\
-82.8^{* * *}\end{array}$ & $\begin{array}{c}1.0 \\
2.8^{* *} \\
31.8^{* *} \\
-35.4^{* *} \\
-85.5^{* * *}\end{array}$ & & $\begin{array}{c}1.2 \\
2.8^{* *} \\
-41.9^{* *} \\
-78.6^{* * *}\end{array}$ \\
\hline [2] & Real house price growth & $\begin{array}{l}\text { Intermediate } \\
\text { Fixed } \\
\Delta \text { FedA/Y } \\
\text { Inter } \times \Delta \text { FedA/Y } \\
\text { Fix } \times \Delta \text { FedA } / Y\end{array}$ & $\begin{array}{l}1.4^{* *} \\
0.8 \\
4.8\end{array}$ & $\begin{array}{c}1.3^{*} \\
1.1 \\
36.0^{*} \\
-7.0 \\
-75.7^{* * *}\end{array}$ & $\begin{array}{c}1.3^{*} \\
1.1 \\
35.1^{*} \\
-9.0 \\
-77.1^{* * *}\end{array}$ & & $\begin{array}{r}0.9 \\
0.7 \\
\\
15.5 \\
-45.6^{*}\end{array}$ \\
\hline [3] & Real stock returns & $\begin{array}{l}\text { Intermediate } \\
\text { Fixed } \\
\Delta \text { FedA/Y } \\
\text { Inter } \times \Delta \text { FedA } / Y \\
\text { Fix } \times \Delta \text { FedA } / Y\end{array}$ & $\begin{array}{c}1.3 \\
0.9 \\
-128.3^{* * *}\end{array}$ & $\begin{array}{c}1.4 \\
1.1 \\
-102.0^{*} \\
-29.5 \\
-57.9\end{array}$ & $\begin{array}{r}0.8 \\
0.2 \\
-82.6 \\
-14.0 \\
-33.0\end{array}$ & & $\begin{aligned} & 2.4^{*} \\
& 2.1 \\
& -17.7 \\
- & 51.4\end{aligned}$ \\
\hline [4] & $\begin{array}{l}\text { Change in loan-to- } \\
\text { deposit ratio }\end{array}$ & $\begin{array}{l}\text { Intermediate } \\
\text { Fixed } \\
\Delta \text { FedA/Y } \\
\text { Inter } \times \Delta \text { FedA/Y } \\
\text { Fix } \times \Delta \text { FedA } / Y\end{array}$ & $\begin{array}{l}0.9 \\
2.3^{*} \\
-7.3\end{array}$ & $\begin{array}{r}0.8 \\
2.3^{*} \\
-9.6 \\
18.8 \\
-13.0\end{array}$ & $\begin{array}{r}0.8 \\
2.5^{*} \\
-12.7 \\
20.3 \\
-14.9\end{array}$ & & $\begin{array}{r}0.6 \\
2.1^{*} \\
\\
15.7 \\
-15.3\end{array}$ \\
\hline [5] & Net capital flows & $\begin{array}{l}\text { Intermediate } \\
\text { Fixed } \\
\Delta \text { FedA } / Y \\
\text { Inter } \times \Delta \text { Fed } A / Y \\
\text { Fix } \times \Delta \text { FedA } / Y\end{array}$ & $\begin{array}{c}2.1^{* *} \\
4.3^{* *} \\
16.6\end{array}$ & $\begin{array}{r}2.2^{* *} \\
4.6^{* *} \\
77.2^{*} \\
-74.1 \\
-86.0\end{array}$ & $\begin{array}{r}2.3^{* * *} \\
5.8^{* * *} \\
80.0^{*} \\
-80.1^{*} \\
-116.1^{*}\end{array}$ & & $\begin{aligned} & 2.6^{* *} \\
& 5.1^{* *} \\
&-83.9^{*} \\
&-92.4\end{aligned}$ \\
\hline [6] & Liability flows & $\begin{array}{l}\text { Intermediate } \\
\text { Fixed } \\
\Delta \text { FedA } / Y \\
\text { Inter } \times \Delta \text { FedA } / Y \\
\text { Fix } \times \Delta \text { FedA } / Y\end{array}$ & $\begin{array}{c}1.0 \\
5.0^{* *} \\
-19.6\end{array}$ & $\begin{array}{c}0.7 \\
4.9^{* *} \\
-44.3 \\
59.7 \\
6.4\end{array}$ & $\begin{array}{c}0.9 \\
6.2^{* * *} \\
-43.1 \\
47.2 \\
-26.7\end{array}$ & & $\begin{array}{l}2.1 \\
6.9^{* * *} \\
44.8 \\
-3.6\end{array}$ \\
\hline [7] & Asset flows & $\begin{array}{l}\text { Intermediate } \\
\text { Fixed } \\
\Delta \text { FedA } / Y \\
\text { Inter } \times \Delta \text { FedA } / Y \\
\text { Fix } \times \Delta \text { FedA } / Y\end{array}$ & $\begin{array}{c}1.1 \\
-0.5 \\
36.2^{* * *}\end{array}$ & $\begin{array}{c}1.5^{*} \\
-0.1 \\
121.8^{* * *} \\
-133.5^{* * *} \\
-92.5^{*}\end{array}$ & $\begin{array}{c}1.4 \\
-0.2 \\
123.4^{* * *} \\
-127.2^{* * *} \\
-89.6^{*}\end{array}$ & & $\begin{array}{c}0.5 \\
-1.6 \\
-128.1^{* * *} \\
-88.8^{*}\end{array}$ \\
\hline [8] & Real GDP growth & $\begin{array}{l}\text { Intermediate } \\
\text { Fixed } \\
\Delta \text { FedA } / Y \\
\text { Inter } \times \Delta \text { FedA } / Y \\
\text { Fix } \times \Delta \text { FedA } / Y\end{array}$ & $\begin{array}{l}0.1 \\
0.1 \\
-4.8^{* *}\end{array}$ & $\begin{array}{r}0.1 \\
0.2 \\
4.1 \\
-4.5 \\
-18.5^{*}\end{array}$ & $\begin{array}{c}0.1 \\
0.2 \\
4.3 \\
-3.9 \\
-18.7^{*}\end{array}$ & & $\begin{array}{c}0.3^{* *} \\
0.4 \\
-6.0 \\
-20.2^{*}\end{array}$ \\
\hline
\end{tabular}

GDP = gross domestic product.

Notes:

1. Only the coefficients of intermediate and fixed exchange rate regimes, $\Delta$ FedA/Y (change in total assets of the Federal Reserve as share of GDP), and their interactions are reported, estimated using propensity score weighted regressions and the same dependent variables as in Table 1, Tables 1.1-1.7, and 1.9, which are listed in the first column.

2. The results in column (4) are not reported because the real shadow funds rate is also included as a regressor.

***, ${ }^{* *},{ }^{*}$ indicate statistical significance at the $1 \%, 5 \%$, and $10 \%$ levels, respectively, based on clustered standard errors (by country).

Source: Author's calculations. 
Table 10 examines the impact of an increase in the expected probability of a recession outside the US. This is analogous to the expected probability of a US recession in the coming quarter considered in Table 4. Again, the results are broadly consistent with our earlier findings. For example, domestic growth, the change in the loan-to-deposit ratio, and asset flows are equally sensitive in countries with intermediate and fixed exchange rate regimes.

In sum, these new results that consider a broader array of measures and constituents of external volatility are consistent with earlier results; namely, that the exchange rate regime still has an effect in accentuating or dampening global volatility. But the results are less consistent with the strong conclusion that only fixed rates significantly diminish volatility relative to the freely floating alternative. Compared with this alternative, intermediate exchange rate regimes also accentuate the impact of real US shocks on a range of financial aggregates in emerging markets. This is also true of the impact of shocks not sourced from the US on not just financial variables in emerging markets but also, in at least one case, on real GDP growth. This is also true for the impact on at least some emerging market financial aggregates of shocks to the federal funds rate and to US unconventional monetary policy.

In some of these cases, it appears that intermediate regimes perform even more poorly than fixed exchange rate regimes in providing insulation. One school of thought, going back to the mid-1990s, is particularly critical of intermediate regimes (Eichengreen 1994, 1999). Intermediate regimes, in this view, are more fragile than hard pegs and free floats, because the commitment of governments to limit the variability of the exchange rate is itself limited. Because of this, an external shock can destabilize the exchange rate regime in addition to destabilizing other domestic real or financial variables. An external shock can force the government and central bank to harden its soft peg or, more likely, to abandon that peg for a freer float. The collapse of the exchange rate regime, actual or anticipated (with some probability), may then further destabilize the other domestic real or financial variables of interest. Intermediate regimes were criticized on these very grounds after the Asian financial crisis-for example, when they showed this kind of fragility and, in the course of doing so, amplified the impact of external volatility on real and financial variables. This is one way of understanding our results suggesting that intermediate regimes, in some cases, have an outside impact on domestic economic and financial conditions.

There are two caveats about this conclusion: First, it is not necessary to defend the strong form bipolar view (Fischer 2001) that pegged and freely floating exchange rates are always better than intermediate regimes to accept some of these conclusions. The point here is not that intermediate regimes are certain to die off or about to disappear, only that they can be fragile and are capable, in some circumstances, of amplifying external disturbances. Second, for some variables and in some specifications, the simple textbook hierarchy that pegged, intermediate, and floating rates provide declining levels of insulation from external disturbances continues to hold. In some cases, however, Obstfeld, Ostry, and Qureshi's (2017) conclusion that mainly pegged rates diminish insulation survives these further robustness checks. 
Table 8: Impact of Non-United States Industrial Production Growth on Various Variables in Emerging Market Economies

\begin{tabular}{|c|c|c|c|c|c|c|c|}
\hline & Dependent Variables & Explained Variables & (1) & (2) & (3) & (4) & (5) \\
\hline [1] & $\begin{array}{l}\text { Real domestic credit } \\
\text { growth }\end{array}$ & $\begin{array}{l}\text { Intermediate } \\
\text { Fixed } \\
\text { NUSIP } \\
\text { Inter } \times \text { NUSIP } \\
\text { Fix } \times \text { NUSIP }\end{array}$ & $\begin{array}{l}1.6^{*} \\
3.2^{* * *} \\
1.6\end{array}$ & $\begin{array}{l}1.5^{*} \\
3.2^{* * *} \\
-0.8 \\
5.7 \\
0.6\end{array}$ & $\begin{array}{c}1.5^{*} \\
3.1^{* *} \\
-1.4 \\
5.9 \\
2.3\end{array}$ & $\begin{array}{l}0.5 \\
3.0^{* *} \\
-1.3 \\
1.5 \\
1.2\end{array}$ & $\begin{array}{l}0.9 \\
2.4^{* *} \\
9.5^{* *} \\
3.1\end{array}$ \\
\hline$[2]$ & Real house price growth & $\begin{array}{l}\text { Intermediate } \\
\text { Fixed } \\
\text { NUSIP } \\
\text { Inter } \times \text { NUSIP } \\
\text { Fix } \times \text { NUSIP }\end{array}$ & $\begin{array}{l}1.4^{* * *} \\
0.9 \\
-8.8\end{array}$ & $\begin{array}{c}1.4^{* * *} \\
1.3 \\
-24.2^{* *} \\
10.6 \\
39.0^{* *}\end{array}$ & $\begin{array}{c}1.4^{* * *} \\
1.3^{*} \\
-24.8^{* *} \\
11.1 \\
39.3^{* *}\end{array}$ & $\begin{array}{c}2.1^{* * *} \\
1.8 \\
-26.7^{* *} \\
12.7 \\
39.7^{* *}\end{array}$ & $\begin{array}{c}1.0^{*} \\
1.0 \\
9.0 \\
37.0^{* *}\end{array}$ \\
\hline [3] & Real stock returns & $\begin{array}{l}\text { Intermediate } \\
\text { Fixed } \\
\text { NUSIP } \\
\text { Inter } \times \text { NUSIP } \\
\text { Fix } \times \text { NUSIP }\end{array}$ & $\begin{array}{c}0.6 \\
-1.6 \\
-36.6^{* * *}\end{array}$ & $\begin{array}{c}0.6 \\
-1.6 \\
-39.9^{* *} \\
4.2 \\
6.1\end{array}$ & $\begin{array}{c}0.3 \\
-2.1 \\
-31.8^{* *} \\
1.8 \\
4.7\end{array}$ & $\begin{array}{r}-0.1 \\
-1.0 \\
-28.0 \\
-4.9 \\
6.4\end{array}$ & $\begin{array}{l}2.6^{* * *} \\
0.2 \\
7.8 \\
12.3\end{array}$ \\
\hline$[4]$ & $\begin{array}{l}\text { Change in loan-to- } \\
\text { deposit ratio }\end{array}$ & $\begin{array}{l}\text { Intermediate } \\
\text { Fixed } \\
\text { NUSIP } \\
\text { Inter } \times \text { NUSIP } \\
\text { Fix } \times \text { NUSIP }\end{array}$ & $\begin{array}{l}1.7^{* *} \\
4.5^{* *} \\
3.0\end{array}$ & $\begin{array}{l}1.6^{* *} \\
4.4^{* *} \\
-5.5 \\
12.5^{*} \\
12.4\end{array}$ & $\begin{array}{l}1.6^{* *} \\
4.4^{* *} \\
-5.6 \\
12.2 \\
12.6\end{array}$ & $\begin{array}{l}1.0 \\
3.9^{* * *} \\
-7.9 \\
10.8 \\
10.2\end{array}$ & $\begin{array}{c}1.0 \\
2.7^{* *} \\
10.8^{*} \\
8.3\end{array}$ \\
\hline [5] & Net capital flows & $\begin{array}{l}\text { Intermediate } \\
\text { Fixed } \\
\text { NUSIP } \\
\text { Inter } \times \text { NUSIP } \\
\text { Fix } \times \text { NUSIP }\end{array}$ & $\begin{array}{l}1.8^{* * *} \\
1.2 \\
1.9\end{array}$ & $\begin{array}{l}1.7^{* * *} \\
1.1 \\
-6.0 \\
9.4 \\
13.7\end{array}$ & $\begin{array}{r}1.7^{* *} \\
1.1 \\
-6.8 \\
9.6 \\
13.6\end{array}$ & $\begin{array}{c}1.3 \\
1.0 \\
-11.6^{*} \\
9.8 \\
11.8\end{array}$ & $\begin{array}{l}2.0^{* *} \\
1.9 \\
15.1^{*} \\
16.7\end{array}$ \\
\hline$[6]$ & Liability flows & $\begin{array}{l}\text { Intermediate } \\
\text { Fixed } \\
\text { NUSIP } \\
\text { Inter } \times \text { NUSIP } \\
\text { Fix } \times \text { NUSIP }\end{array}$ & $\begin{array}{c}0.9 \\
2.1 \\
15.7^{\star *}\end{array}$ & $\begin{array}{c}1.0 \\
2.0 \\
18.5^{* *} \\
-12.1 \\
5.4\end{array}$ & $\begin{array}{c}0.9 \\
1.9 \\
18.7^{* *} \\
-13.3 \\
4.1\end{array}$ & $\begin{array}{r}-0.5 \\
1.5 \\
8.1 \\
-13.9^{*} \\
1.5\end{array}$ & $\begin{array}{l}1.9^{* *} \\
3.6^{*} \\
\\
2.1 \\
12.7\end{array}$ \\
\hline [7] & Asset flows & $\begin{array}{l}\text { Intermediate } \\
\text { Fixed } \\
\text { NUSIP } \\
\text { Inter } \times \text { NUSIP } \\
\text { Fix } \times \text { NUSIP }\end{array}$ & $\begin{array}{c}0.9 \\
-0.8 \\
-13.9^{* * *}\end{array}$ & $\begin{array}{c}0.7 \\
-0.9 \\
-24.6^{* * *} \\
21.5^{* * *} \\
8.1\end{array}$ & $\begin{array}{c}0.8 \\
-0.8 \\
-25.6^{\text {***}} \\
22.9^{* * *} \\
9.3\end{array}$ & $\begin{array}{c}1.7 \\
-0.4 \\
-19.9^{* * *} \\
23.6^{* * *} \\
9.9\end{array}$ & $\begin{array}{c}0.0 \\
-1.6^{*} \\
13.0 \\
3.4\end{array}$ \\
\hline [8] & Real GDP growth & $\begin{array}{l}\text { Intermediate } \\
\text { Fixed } \\
\text { NUSIP } \\
\text { Inter } \times \text { NUSIP } \\
\text { Fix } \times \text { NUSIP }\end{array}$ & $\begin{array}{l}0.1 \\
0.4^{*} \\
5.0^{* * *}\end{array}$ & $\begin{array}{l}0.1 \\
0.3 \\
3.0^{* *} \\
-0.1 \\
6.4^{*}\end{array}$ & $\begin{array}{l}0.1 \\
0.3 \\
3.5^{* *} \\
-0.3 \\
6.3^{*}\end{array}$ & $\begin{array}{l}0.1 \\
0.4 \\
3.6^{* * *} \\
-0.4 \\
6.3^{*}\end{array}$ & $\begin{array}{c}0.1 \\
0.2 \\
-0.5 \\
6.2^{* *}\end{array}$ \\
\hline
\end{tabular}

GDP = gross domestic product, NUSIP = non-United States industrial production.

Notes: Only the coefficients of intermediate and fixed exchange rate regimes, non-US industrial production growth, and their interactions are reported, estimated using propensity score weighted regressions and the same dependent variables as in Table 1, Tables 1.1-1.7, and 1.9, which are listed in the first column.

***, **, ${ }^{*}$ indicate statistical significance at the $1 \%, 5 \%$, and $10 \%$ levels, respectively, based on clustered standard errors (by country).

Source: Author's calculations. 


\section{Table 9: Impact of the Global Economic Policy Uncertainty Index on Various Variables in Emerging Market Economies}

\begin{tabular}{|c|c|c|c|c|c|c|c|}
\hline & Dependent Variables & Explained Variables & (1) & (2) & (3) & (4) & (5) \\
\hline$[1]$ & $\begin{array}{l}\text { Real domestic credit } \\
\text { growth }\end{array}$ & $\begin{array}{l}\text { Intermediate } \\
\text { Fixed } \\
\text { GEPU } \\
\text { Inter } \times \text { GEPU } \\
\text { Fix } \times \text { GEPU }\end{array}$ & $\begin{array}{c}0.8 \\
1.6^{*} \\
-2.1^{* * *}\end{array}$ & $\begin{array}{l}5.4 \\
14.0^{* *} \\
-1.0 \\
-1.0 \\
-2.7^{*}\end{array}$ & $\begin{array}{l}4.9 \\
16.0^{* *} \\
-0.8 \\
-0.9 \\
-3.2^{* *}\end{array}$ & $\begin{array}{l}2.6 \\
25.4^{* * *} \\
-0.2 \\
-0.5 \\
-4.9^{* * *}\end{array}$ & $\begin{array}{c}4.7 \\
14.5^{* *} \\
-0.9 \\
-2.8^{* *}\end{array}$ \\
\hline$[2]$ & Real house price growth & $\begin{array}{l}\text { Intermediate } \\
\text { Fixed } \\
\text { GEPU } \\
\text { Inter } \times \text { GEPU } \\
\text { Fix } \times \text { GEPU }\end{array}$ & $\begin{array}{l}0.9^{* *} \\
0.7 \\
-1.9^{* * *}\end{array}$ & $\begin{array}{l}-6.6 \\
10.3^{* *} \\
-1.7^{* * *} \\
1.7 \\
-2.0^{* *}\end{array}$ & $\begin{array}{l}-6.7 \\
10.9^{* *} \\
-1.6^{* * *} \\
1.7 \\
-2.1^{* *}\end{array}$ & $\begin{array}{l}-7.3^{* *} \\
11.5 \\
-1.8^{* * *} \\
1.8^{* * *} \\
-2.3\end{array}$ & $\begin{array}{r}-12.0 \\
3.8 \\
\\
2.9 \\
-0.6\end{array}$ \\
\hline$[3]$ & Real stock returns & $\begin{array}{l}\text { Intermediate } \\
\text { Fixed } \\
\text { GEPU } \\
\text { Inter } \times \text { GEPU } \\
\text { Fix } \times \text { GEPU }\end{array}$ & $\begin{array}{c}1.4^{*} \\
-1.2 \\
-9.8^{* * *}\end{array}$ & $\begin{array}{l}8.1 \\
-7.5 \\
-9.8^{* * *} \\
-1.4 \\
1.4\end{array}$ & $\begin{array}{c}7.8 \\
-6.5 \\
-10.2^{\text {***}}-1.3 \\
1.1\end{array}$ & $\begin{array}{c}7.5 \\
0.9 \\
-15.1^{* * *} \\
-1.3 \\
-0.3\end{array}$ & $\begin{array}{r}7.2 \\
-10.3 \\
\\
-1.0 \\
2.3\end{array}$ \\
\hline$[4]$ & $\begin{array}{l}\text { Change in loan-to- } \\
\text { deposit ratio }\end{array}$ & $\begin{array}{l}\text { Intermediate } \\
\text { Fixed } \\
\text { GEPU } \\
\text { Inter } \times \text { GEPU } \\
\text { Fix } \times \text { GEPU }\end{array}$ & $\begin{array}{r}0.4 \\
1.2 \\
-0.7\end{array}$ & $\begin{array}{r}4.8 \\
8.0 \\
0.0 \\
-1.0 \\
-1.5\end{array}$ & $\begin{array}{c}4.3 \\
8.7^{*} \\
0.1 \\
-0.9 \\
-1.7^{*}\end{array}$ & $\begin{array}{c}3.9 \\
14.5^{* *} \\
1.0 \\
-0.8 \\
-2.8^{* *}\end{array}$ & $\begin{array}{c}4.4 \\
8.5^{*} \\
-0.9 \\
-1.6^{*}\end{array}$ \\
\hline$[5]$ & Net capital flows & $\begin{array}{l}\text { Intermediate } \\
\text { Fixed } \\
\text { GEPU } \\
\text { Inter } \times \text { GEPU } \\
\text { Fix } \times \text { GEPU }\end{array}$ & $\begin{array}{l}1.9^{* * *} \\
1.3 \\
-2.7^{* *}\end{array}$ & $\begin{array}{l}17.6^{* *} \\
28.9^{*} \\
0.2 \\
-3.5^{* *} \\
-6.0^{*}\end{array}$ & $\begin{array}{l}17.5^{* *} \\
28.5^{*} \\
0.2 \\
-3.5^{* *} \\
-5.9^{*}\end{array}$ & $\begin{array}{l}15.1^{* *} \\
35.1^{* * *} \\
0.8 \\
-3.0^{*} \\
-7.2^{* * *}\end{array}$ & $\begin{array}{l}14.9^{*} \\
28.1^{*} \\
-2.8 \\
-5.7^{*}\end{array}$ \\
\hline$[6]$ & Liability flows & $\begin{array}{l}\text { Intermediate } \\
\text { Fixed } \\
\text { GEPU } \\
\text { Inter } \times \text { GEPU } \\
\text { Fix } \times \text { GEPU }\end{array}$ & $\begin{array}{c}1.3^{* *} \\
2.8^{*} \\
-6.5^{* * *}\end{array}$ & $\begin{array}{l}14.6 \\
33.2^{* *} \\
-3.5^{* * *} \\
-3.0 \\
-6.6^{*}\end{array}$ & $\begin{array}{l}11.9 \\
31.6^{* *} \\
-3.8^{* * *} \\
-2.4 \\
-6.2^{*}\end{array}$ & $\begin{array}{l}5.1 \\
37.5^{* * *} \\
-2.6^{*} \\
-1.1 \\
-7.4^{* *}\end{array}$ & $\begin{array}{l}13.7 \\
34.4^{*} \\
-2.6 \\
-6.6^{*}\end{array}$ \\
\hline$[7]$ & Asset flows & $\begin{array}{l}\text { Intermediate } \\
\text { Fixed } \\
\text { GEPU } \\
\text { Inter } \times \text { GEPU } \\
\text { Fix } \times \text { GEPU }\end{array}$ & $\begin{array}{l}0.6 \\
-1.4 \\
3.7^{* * *}\end{array}$ & $\begin{array}{l}3.5 \\
-4.0 \\
3.7^{\text {***}} \\
-0.6 \\
0.6\end{array}$ & $\begin{array}{l}6.1 \\
-2.8 \\
4.0^{* * *} \\
-1.2 \\
0.3\end{array}$ & $\begin{array}{l}10.4^{*} \\
-2.0 \\
3.4^{* * *} \\
-1.9 \\
0.2\end{array}$ & $\begin{array}{r}1.5 \\
-6.1 \\
-0.3 \\
0.9\end{array}$ \\
\hline$[8]$ & Real GDP growth & $\begin{array}{l}\text { Intermediate } \\
\text { Fixed } \\
\text { GEPU } \\
\text { Inter } \times \text { GEPU } \\
\text { Fix } \times \text { GEPU }\end{array}$ & $\begin{array}{l}0.0 \\
0.2 \\
-0.8^{* * *}\end{array}$ & $\begin{array}{l}0.1 \\
1.6 \\
-0.7^{\text {***}} \\
-0.0 \\
-0.3\end{array}$ & $\begin{array}{l}0.3 \\
1.6 \\
-0.7^{* * *} \\
-0.0 \\
-0.3\end{array}$ & $\begin{array}{l}1.5 \\
0.9 \\
-0.7^{* * *} \\
-0.3 \\
-0.1\end{array}$ & $\begin{array}{r}0.2 \\
1.8 \\
-0.0 \\
-0.3\end{array}$ \\
\hline
\end{tabular}

GDP = gross domestic product, GEPU = Global Economic Policy Uncertainty Index.

Notes: Only the coefficients of intermediate and fixed exchange rate regimes, the Global Economic Policy Uncertainty Index, and their interactions are reported, estimated using propensity score weighted regressions and the same dependent variables as in Table 1, Tables 1.1-1.7, and 1.9, which are listed in the first column.

***, **, * indicate statistical significance at the 1\%, $5 \%$, and $10 \%$ levels, respectively, based on clustered standard errors (by country).

Source: Author's calculations. 
Table 10: Impact of the Expected Probability of Recessions Outside the United States on Various Variables in Emerging Market Economies

\begin{tabular}{|c|c|c|c|c|c|c|c|}
\hline & Dependent Variables & Explained Variables & (1) & (2) & (3) & (4) & (5) \\
\hline [1] & $\begin{array}{l}\text { Real domestic credit } \\
\text { growth }\end{array}$ & $\begin{array}{l}\text { Intermediate } \\
\text { Fixed } \\
\text { EPROUS } \\
\text { Inter } \times \text { EPROUS } \\
\text { Fix } \times \text { EPROUS }\end{array}$ & $\begin{array}{l}1.56^{*} \\
3.13^{* *} \\
-0.96\end{array}$ & $\begin{array}{l}1.87^{* *} \\
3.49^{* * *} \\
0.53 \\
-1.86^{*} \\
-2.38^{*}\end{array}$ & $\begin{array}{l}1.86^{* *} \\
3.41^{* *} \\
0.40 \\
-2.11^{*} \\
-2.13\end{array}$ & $\begin{array}{l}0.71 \\
3.27^{* *} \\
0.53 \\
-2.08^{*} \\
-2.55^{*}\end{array}$ & $\begin{array}{l}1.46^{*} \\
2.86^{* * *} \\
-2.26^{*} \\
-2.26^{*}\end{array}$ \\
\hline$[2]$ & Real house price growth & $\begin{array}{l}\text { Intermediate } \\
\text { Fixed } \\
\text { EPROUS } \\
\text { Inter } \times \text { EPROUS } \\
\text { Fix } \times \text { EPROUS }\end{array}$ & $\begin{array}{l}1.41^{* * *} \\
1.26 \\
-1.60\end{array}$ & $\begin{array}{l}1.92^{* *} \\
3.18^{* *} \\
1.98 \\
-1.74 \\
-6.71^{* *}\end{array}$ & $\begin{array}{l}1.93^{* *} \\
3.37^{* *} \\
2.09 \\
-2.03 \\
-7.09^{* *}\end{array}$ & $\begin{array}{l}1.62^{* * *} \\
2.31 \\
1.99 \\
-1.79 \\
-6.81^{* *}\end{array}$ & $\begin{array}{c}1.17 \\
2.36^{*} \\
\\
0.65 \\
-5.02\end{array}$ \\
\hline [3] & Real stock returns & $\begin{array}{l}\text { Intermediate } \\
\text { Fixed } \\
\text { EPROUS } \\
\text { Inter } \times \text { EPROUS } \\
\text { Fix } \times \text { EPROUS }\end{array}$ & $\begin{array}{c}-1.00 \\
-4.31^{* *} \\
-14.99^{* * *}\end{array}$ & $\begin{array}{l}-1.64 \\
-3.95^{* *} \\
-15.52^{* * *} \\
3.64 \\
-2.72\end{array}$ & $\begin{array}{c}-1.83 \\
-4.13^{* *} \\
-14.51^{* * *} \\
4.52 \\
-1.64\end{array}$ & $\begin{array}{l}-1.39 \\
-3.94^{* *} \\
-15.14^{* * *} \\
3.61 \\
-2.78\end{array}$ & $\begin{array}{l}2.45^{* *} \\
0.73 \\
\\
1.20 \\
-4.19\end{array}$ \\
\hline [4] & $\begin{array}{l}\text { Change in loan-to- } \\
\text { deposit ratio }\end{array}$ & $\begin{array}{l}\text { Intermediate } \\
\text { Fixed } \\
\text { EPROUS } \\
\text { Inter } \times \text { EPROUS } \\
\text { Fix } \times \text { EPROUS }\end{array}$ & $\begin{array}{l}1.82^{* *} \\
4.51^{* *} \\
0.48\end{array}$ & $\begin{array}{l}2.14^{* *} \\
4.75^{* * *} \\
1.59^{* * *} \\
-1.94^{* *} \\
-1.51^{* * *}\end{array}$ & $\begin{array}{l}2.15^{* *} \\
4.75^{* * *} \\
1.72^{* * *} \\
-2.28^{* *} \\
-1.69^{* *}\end{array}$ & $\begin{array}{l}1.21 \\
3.92^{* * *} \\
1.54^{* * *} \\
-2.15^{* *} \\
-1.69^{* * *}\end{array}$ & $\begin{array}{l}1.63^{* *} \\
3.12^{* *} \\
-2.65^{* * *} \\
-1.75^{* * *}\end{array}$ \\
\hline$[5]$ & Net capital flows & $\begin{array}{l}\text { Intermediate } \\
\text { Fixed } \\
\text { EPROUS } \\
\text { Inter } \times \text { EPROUS } \\
\text { Fix } \times \text { EPROUS }\end{array}$ & $\begin{array}{c}1.77^{* * *} \\
1.21 \\
-0.45\end{array}$ & $\begin{array}{l}1.82^{* *} \\
1.23 \\
-0.30 \\
-0.28 \\
-0.19\end{array}$ & $\begin{array}{l}1.81^{* *} \\
1.25 \\
-0.41 \\
-0.44 \\
-0.52\end{array}$ & $\begin{array}{r}1.21 \\
0.70 \\
-0.41 \\
-0.60 \\
-0.29\end{array}$ & $\begin{array}{l}2.65^{* * *} \\
2.38 \\
-2.33 \\
-1.36\end{array}$ \\
\hline$[6]$ & Liability flows & $\begin{array}{l}\text { Intermediate } \\
\text { Fixed } \\
\text { EPROUS } \\
\text { Inter } \times \text { EPROUS } \\
\text { Fix } \times \text { EPROUS }\end{array}$ & $\begin{array}{r}0.61 \\
1.70 \\
-1.79\end{array}$ & $\begin{array}{l}-0.02 \\
1.34 \\
-3.68^{* *} \\
3.56^{* *} \\
2.40\end{array}$ & $\begin{array}{l}-0.02 \\
1.38 \\
-3.99^{* * *} \\
3.24^{*} \\
2.06\end{array}$ & $\begin{array}{l}-0.80 \\
0.81 \\
-4.47^{* * *} \\
3.26^{* *} \\
2.39\end{array}$ & $\begin{array}{l}1.74 \\
3.60^{*} \\
1.37 \\
1.29\end{array}$ \\
\hline [7] & Asset flows & $\begin{array}{l}\text { Intermediate } \\
\text { Fixed } \\
\text { EPROUS } \\
\text { Inter } \times \text { EPROUS } \\
\text { Fix } \times \text { EPROUS }\end{array}$ & $\begin{array}{c}1.16^{*} \\
-0.43 \\
1.34^{* *}\end{array}$ & $\begin{array}{l}1.83^{* *} \\
-0.02 \\
3.38^{* * *} \\
-3.82^{* * *} \\
-2.60^{*}\end{array}$ & $\begin{array}{l}1.81^{* *} \\
-0.06 \\
3.60^{* * *} \\
-3.66^{* * *} \\
-2.58^{*}\end{array}$ & $\begin{array}{l}1.99^{*} \\
-0.00 \\
4.06^{* * *} \\
-3.84^{* * *} \\
-2.67^{*}\end{array}$ & $\begin{array}{l}0.89 \\
-1.13 \\
-3.67^{* * *} \\
-2.65^{*}\end{array}$ \\
\hline$[8]$ & Real GDP growth & $\begin{array}{l}\text { Intermediate } \\
\text { Fixed } \\
\text { EPROUS } \\
\text { Inter } \times \text { EPROUS } \\
\text { Fix } \times \text { EPROUS }\end{array}$ & $\begin{array}{c}0.04 \\
0.28 \\
-0.61^{* * *}\end{array}$ & $\begin{array}{l}0.03 \\
0.28 \\
-0.62^{* * *} \\
0.02 \\
-0.00\end{array}$ & $\begin{array}{l}0.03 \\
0.27 \\
-0.65^{* * *} \\
0.13 \\
0.06\end{array}$ & $\begin{array}{l}0.08 \\
0.20 \\
-0.66^{* * *} \\
0.02 \\
-0.04\end{array}$ & $\begin{array}{l}0.07 \\
0.30 \\
-0.00 \\
-0.12\end{array}$ \\
\hline
\end{tabular}

EPROUS = expected probability of recessions outside the United States, GDP =gross domestic product.

Notes:

1. Only the coefficients of intermediate and fixed exchange rate regimes, the expected probability of recessions outside of the United States, and their interactions are reported, estimated using propensity score weighted regressions and the same dependent variables as in Table 1, Tables 1.1-1.7, and 1.9, which are listed in the first column.

2. The expected probability of recessions outside of the United States within the next 12 months is calculated by Federal Reserve Board staff using excess bond premium and the global condition index. The authors thank Andrea Raffo for providing the estimates.

$* * *, * *,{ }^{*}$ indicate statistical significance at the $1 \%, 5 \%$, and $10 \%$ levels, respectively, based on clustered standard errors by country). Source: Author's calculations. 


\section{CONCLUSION}

Recent literature on the global financial cycle, the role of the dollar in bank funding markets, and the role of dollar invoicing and settlements have raised anew old questions about the insulating properties of flexible exchange rates in general and for emerging markets in particular. Some studies that respond to this question marshal evidence that more flexible exchange rates do in fact provide insulation-and that countries with pegged exchange rates experience more domestic volatility in response to global shocks. The principal these studies produce also leads to a further strong conclusion: that countries sacrifice the insulating properties of flexible exchange rates only when they move all the way to the other extreme-that is, to a currency peg-and not when they move only part way to intermediate regimes of managed flexibility.

Clearly, the stakes are high, for emerging markets in general and Asian ones in particular. Global volatility is a problem for these countries. Whether the exchange rate regime can be tailored to limit the domestic impact of that volatility is an important policy question. The subsidiary question then becomes how exactly the exchange rate regime should be tailored. Asian countries have extensive experience with global volatility and its effects. And they have received much advice, in the last 2 decades in particular, about how to tailor their exchange rate regimes.

Our empirical analysis revisits these questions, building directly on the recent literature, but using alternative measures of global volatility and alternative empirical methods. We come down on the side that the exchange rate regime matters, and that more flexible exchange rates provide emerging markets with more insulation from global volatility shocks. Here, our results are consistent with the conventional wisdom and with the findings of Obstfeld, Ostry, and Qureshi (2017), and inconsistent with the theory of the global financial cycle and related analyses that deny the existence of those insulating properties. Our results provide some support for the notion that both fixed and intermediate exchange rate regimes diminish insulation relative to the alternative of fully flexible rates. In some cases, intermediate regimes accentuate the impact of global volatility on domestic financial variables as much as pegged regimes.

According to Obstfeld, Ostry, and Qureshi (2017), limited flexibility was enough if policy makers want to avoid accentuating the impact of global volatility on domestic conditions through their choice of exchange rate regime. But our findings suggest this is not always the case. To this end, we would also like to emphasize that despite the insulation properties of flexible exchange rate regimes, adopting them must be strongly supported by a domestic economic environment that is conducive for relatively stable currency movements. These are important for promoting healthy domestic credit conditions, asset price development, capital flows, and GDP growth. So, while a stable exchange rate environment promotes the development of domestic financial conditions, its potential to move flexibly in responding to external shocks will help an economy to insulate the unwanted effects of these shocks. 


\section{APPENDIXES}

Table A.1: Description and Sources for the Variables Used by Obstfeld, Ostry, and Qureshi

\begin{tabular}{|c|c|c|}
\hline Variables & Description & Source \\
\hline Capital account openness & Index (high = liberalized, low = closed $)$ & Quinn and Toyoda (2008) \\
\hline Capital flows & $\begin{array}{l}\text { In billions of dollars (BPM5 presentation). Net financial } \\
\text { flows exclude financing items and other investment } \\
\text { liabilities of general government; i.e., the difference } \\
\text { between the International Monetary Fund's International } \\
\text { Financial Statistics (IFS) series codes “... 4995W.9" and “... } \\
\text { 4753ZB9." Liability flows and other investment liability } \\
\text { flows also exclude other investment liabilities of the } \\
\text { general government }\end{array}$ & IFS database \\
\hline Consumer price index (CPI) & Index & IFS database \\
\hline $\begin{array}{l}\text { Domestic private sector } \\
\text { credit }\end{array}$ & In local currency & IFS database \\
\hline Exchange rate regime & De facto, de jure & $\begin{array}{l}\text { Ghosh, Ostry, and Qureshi } \\
\text { (2015) and Reinhart and Rogoff } \\
\text { (2004) updated data from } \\
\text { http://personal.Ise.ac.uk/ilzetzki/ } \\
\text { index.htm/Data.htm. }\end{array}$ \\
\hline $\begin{array}{l}\text { Gross domestic product } \\
\text { current and constant } \\
\text { prices }\end{array}$ & $\begin{array}{l}\text { In billions of dollars (or local currency); seasonally } \\
\text { adjusted observations for quarterly data }\end{array}$ & $\begin{array}{l}\text { Haver Analytics, CEIC, and IFS } \\
\text { database }\end{array}$ \\
\hline House prices & Index (in real terms) & $\begin{array}{l}\text { Bank for International } \\
\text { Settlements }\end{array}$ \\
\hline Institutional quality & $\begin{array}{l}\text { Index (average of International Country Risk Guide's } \\
\text { [ICRG] } 12 \text { political risk components) }\end{array}$ & Political Risk Group \\
\hline Loan-to-deposit ratio & In percent & IFS database \\
\hline Policy rate & Policy rate or discount rate (in percent) & IFS database \\
\hline Reserve requirements & $\begin{array}{l}\text { Average of reserve requirements on local currency } \\
\text { demand, saving, and term deposits (in percent) }\end{array}$ & $\begin{array}{l}\text { Authors' calculations based on } \\
\text { data from Federico et al. (2014) }\end{array}$ \\
\hline Shadow federal funds rate & $\begin{array}{l}\text { In percent. In real terms computed as }[(1+\text { nominal interest } \\
\text { rate }) /(1+\text { expected inflation })]-1 \text {, where expected inflation } \\
\text { is one-period ahead inflation }\end{array}$ & Federal Reserve Economic Data \\
\hline Stock prices (in real terms) & Stock price index deflated by quarterly CPI & CEIC and author's calculations \\
\hline $\begin{array}{l}\text { United States (US) interest } \\
\text { rate }\end{array}$ & $\begin{array}{l}\text { US 3-month Treasury bill rate and 10-year government } \\
\text { bond yield (in percent) }\end{array}$ & IFS database and Bloomberg \\
\hline VXO and VIX Indexes & Chicago Board Options Exchange Market Volatility Index & Bloomberg \\
\hline
\end{tabular}

Notes:

1. Description and source of all variables not shaded are identical to those in Obstfeld, Ostry, and Qureshi (2017). Since proprietary data series are not provided by these authors, we have collected these data.

2. Except for institutional quality, proxied by the ICRG, which is collected from the same source as that in Obstfeld, Ostry, and Qureshi (2017), other data denoted by shade are collected from different sources.

3. Although CPI and the shadow federal funds rate are not proprietary data, since they were not provided by Obstfeld, Ostry, and Qureshi, the compilers of this table collected them from publicly available sources.

Sources: Authors' compilation based on Federico, Pablo, Carlos Vegh, and Guillermo Vuletin. 2014. "Reserve Requirements over the Business Cycle." NBER Working Paper No. 20612. Cambridge, MA: National Bureau of Economic Research; Ghosh, Atish, Jonathan Ostry, and Mahvesh Qureshi. 2015. "Exchange Rate Management and Crisis Susceptibility: A Reassessment." IMF Economic Review 63: 238-76; Obstfeld, Maurice, Jonathan Ostry, and Mahvash Qureshi. 2017. "A Tie That Binds: Revisiting the Trilemma in Emerging Market Economies." IMF Working Paper WP/17/130. Washington, DC: International Monetary Fund; Reinhart, Carmen, and Kenneth Rogoff. 2004. "The Modern History of Exchange Rate Arrangements: A Reinterpretation." Quarterly Journal of Economics 199: 1-48; Quinn, Dennis, and Maria Toyoda. 2008. “Does Capital Account Liberalization Lead to Economic Growth?” Review of Financial Studies 21 (3): 1403-49. 
Table A.2: Description and Sources for the Variables Used by Londono and Wilson

\begin{tabular}{|c|c|c|}
\hline Variables & Description & Source \\
\hline $\begin{array}{l}\text { Londono and Wilson } \\
\text { index }\end{array}$ & $\begin{array}{l}\text { Market-value-weighted average of the equity option- } \\
\text { implied volatility for seven countries: France, Germany, } \\
\text { Japan, the Netherlands, Switzerland, the United Kingdom, } \\
\text { and the United States (US) }\end{array}$ & $\begin{array}{l}\text { Chicago Board Options } \\
\text { Exchange; Datastream; Osaka } \\
\text { University, Center for the Study } \\
\text { of Finance and Insurance }\end{array}$ \\
\hline $\begin{array}{l}\text { US Industrial } \\
\text { Production Index }\end{array}$ & $\begin{array}{l}\text { Industrial Production Index, index } 2012=100 \text {, quarterly } \\
\text { average of monthly data, seasonally adjusted }\end{array}$ & Federal Reserve Economic Data \\
\hline $\begin{array}{l}\text { Expected probability of } \\
\text { a US recession }\end{array}$ & $\begin{array}{l}\text { Probability that real gross national product/GDP will decline } \\
\text { (quarter over quarter) in the following quarter the survey } \\
\text { was conducted }\end{array}$ & $\begin{array}{l}\text { Survey of Professional } \\
\text { Forecasters }\end{array}$ \\
\hline $\begin{array}{l}\text { US Economic Policy } \\
\text { Uncertainty Index }\end{array}$ & $\begin{array}{l}\text { Economic Policy Uncertainty Index, seasonally adjusted, } \\
\text { following definition in Baker, Bloom, and Davis (2016) }\end{array}$ & Federal Reserve Economic Data \\
\hline Federal funds rate & $\begin{array}{l}\text { Fed funds rate when this rate is above zero and the shadow } \\
\text { fed funds rate (Wu and Xia 2016) in the period when the } \\
\text { zero-lower bound is binding }\end{array}$ & $\begin{array}{l}\text { Normal time rate: Federal } \\
\text { Reserve Economic Data; shadow } \\
\text { rate: Wu and Xia (2016) }\end{array}$ \\
\hline $\begin{array}{l}\text { Change in Federal } \\
\text { Reserve assets as a } \\
\text { share of US gross } \\
\text { domestic product } \\
\text { (GDP) }\end{array}$ & $\begin{array}{l}\text { Change in total assets of all Federal Reserve Banks } \\
\text { (quarterly average data) divided by GDP (annual data), } \\
\text { seasonally adjusted }\end{array}$ & $\begin{array}{l}\text { Total Federal Reserve assets: } \\
\text { Federal Reserve Economic Data; } \\
\text { GDP: US Bureau of Economic } \\
\text { Analysis, retrieved from Federal } \\
\text { Reserve Economic Data }\end{array}$ \\
\hline $\begin{array}{l}\text { Non-US industrial } \\
\text { production growth }\end{array}$ & $\begin{array}{l}\text { Industrial production, seasonally adjusted, index } \\
\text { Average of six countries: France, Germany, Japan, the } \\
\text { Netherlands, Switzerland, and the United Kingdom }\end{array}$ & $\begin{array}{l}\text { International Monetary Fund's } \\
\text { (IMF) International Financial } \\
\text { Statistics database; Federal } \\
\text { Reserve Economic Data }\end{array}$ \\
\hline $\begin{array}{l}\text { Global Economic } \\
\text { Policy Uncertainty } \\
\text { Index }\end{array}$ & $\begin{array}{l}\text { GDP-weighted average of the } 18 \text { national Economic Policy } \\
\text { Uncertainty Index values, using GDP data from the World } \\
\text { Economic Outlook database of the IMF }\end{array}$ & $\begin{array}{l}\text { Global Economic Policy } \\
\text { Uncertainty Index } \\
\text { (www.PolicyUncertainty.com) }\end{array}$ \\
\hline $\begin{array}{l}\text { Expected probability of } \\
\text { recessions outside the } \\
\text { US }\end{array}$ & $\begin{array}{l}\text { The expected probability of recessions outside the US } \\
\text { within the next } 12 \text { months, calculated by the Federal } \\
\text { Reserve Board staff using excess bond premium and Global } \\
\text { Conditions Index }\end{array}$ & $\begin{array}{l}\text { Cuba-Borda, Mechanik, and } \\
\text { Raffo (2018) }\end{array}$ \\
\hline
\end{tabular}

Sources: Authors' compilation based on Londono, Juan, and Beth Anne Wilson. 2018. "Understanding Global Volatility." IFDP Notes. Washington, DC: Board of Governors of the Federal Reserve System; Baker, Scott R., Nicholas Bloom, and Steven J. Davis. 2016. "Measuring Economic Policy Uncertainty." Quarterly Journal of Economics 131 (4): 1593-636; Cuba-Borda, Pablo, Alexander Mechanik, and Andrea Raffo. 2018. "Monitoring the World Economy: A Global Condition Index." IFDP Notes. Washington, DC: Board of Governors of the Federal Reserve System; Wu, Jing Cynthia, and Fan Dora Xia. 2016. "Measuring the Macroeconomic Impact of Monetary Policy at the Zero Lower Bound." Journal of Money, Credit and Banking 48 (2-3): 253-91. 


\section{REFERENCES}

Accominotti, Olivier, and Barry Eichengreen. 2016. "The Mother of All Sudden Stops: Capital Flows and Reversals, in Europe, 1919-32.” Economic History Review 69 (2): 469-92.

Aizenman, Joshua, Menzie Chinn, and Hiro Ito. 2015. "Monetary Policy Spillovers and the Trilemma in the New Normal: Periphery Country Sensitivity to Core Country Conditions." NBER Working Paper No. 21128. Cambridge, MA: National Bureau of Economic Research.

Baker, Scott R., Nicholas Bloom, and Steven J. Davis. 2016. "Measuring Economic Policy Uncertainty.” Quarterly Journal of Economics 131 (4): 1593-636.

Bank for International Settlements. https://www.bis.org/ (accessed 18 August 2018).

Baxter, Marianne, and Alan Stockman. 1989. "Business Cycles and the Exchange-Rate Regime: Some International Evidence.” Journal of Monetary Economics 23 (3): 377-400.

Bekaert, Geert, and Arnaud Mehl. 2017. "On the Global Financial Market Integration 'Swoosh' and the Trilemma.” NBER Working Paper No. 23124. Cambridge, MA: National Bureau of Economic Research.

Bluedorn, John C., and Christopher Bowdler. 2010. "The Empirics of International Monetary Transmission: Identification and the Impossible Trinity." Journal of Money, Credit and Banking 42 (4): 679-713.

Caceres, Carlos, Yan Carriere-Swallow, and Bertrand Gruss. 2016. "Global Financial Conditions and Monetary Policy Autonomy.” IMF Working Paper WP/16/108. Washington, DC: International Monetary Fund.

Choudhri, Ehsan, and Levis Kochin. 1980. "The Exchange Rate and the International Transmission of Business Cycle Disturbances: Some Evidence from the Great Depression." Journal of Money, Credit and Banking 12 (4): 565-74.

Cuba-Borda, Pablo, Alexander Mechanik, and Andrea Raffo. 2018. "Monitoring the World Economy: A Global Condition Index." IFDP Notes. Washington, DC: Board of Governors of the Federal Reserve System.

Cushman, David, and Glauco De Vita. 2017. "Exchange Rate Regimes and FDI in Developing Countries: A Propensity Score Matching Approach." Journal of International Money and Finance 77 (C): 143-63.

Edwards, Sebastian. 2015. "Monetary Policy Independence under Flexible Exchange Rates: An Illusion?" World Economy 38 (5): 773-87.

Eichengreen, Barry. 1992. "The Comparative Performance of Fixed and Flexible Exchange Rate Regimes: Interwar Evidence." In Business Cycles, Theories, Evidence and Analysis, edited by Niels Thygesen, Kumaraswamy Velupillai, and Stefano Zambelli, 229-72. New York: St. Martin's Press. 
1994. International Monetary Arrangements for the 21st Century. Washington, DC: Brookings Institution.

1999. Toward a New International Financial Architecture: A Practical Post-Asia Agenda. Washington, DC: Institute of International Economics.

Eichengreen, Barry, and Jeffrey Sachs. 1985. "Exchange Rates and Economic Recovery in the 1930s." Journal of Economic History 55 (4): 925-46.

Federico, Pablo, Carlos Vegh, and Guillermo Vuletin. 2014. "Reserve Requirements over the Business Cycle.” NBER Working Paper No. 20612. Cambridge, MA: National Bureau of Economic Research.

Fischer, Stanley. 2001. "Exchange Rate Regimes: Is the Bipolar View Correct?" Journal of Economic Perspectives 15 (2): 3-24.

Frankel, Jeffrey, Sergio Schmukler, and Luis Serven. 2004. "Global Transmission of Interest Rates: Monetary Independence and Currency Regime." Journal of International Money and Finance 23 (95): 701-33.

Georgiadis, Georgios, and Arnaud Mehl. 2015. "Trilemma, Not Dilemma: Financial Globalization and Monetary Policy Effectiveness." Globalization and Monetary Policy Institute Working Paper No. 222. Dallas: Federal Reserve Bank of Dallas.

Ghosh, Atish, Jonathan Ostry, and Mahvesh Qureshi. 2015. "Exchange Rate Management and Crisis Susceptibility: A Reassessment.” IMF Economic Review 63: 238-76.

Gopinath, Gita. 2017a. "The International Price System.” Proceedings of the Jackson Hole Symposium. Kansas City: Federal Reserve Bank of Kansas City. 2017b. “Rethinking International Macroeconomic Policy.” Unpublished.

International Monetary Fund. "De Facto Classification of Exchange Rate Regimes and Monetary Policy Framework." https:/www.imf.org/external/np/mfd/er/2006/eng/0706.htm. International Financial Statistics. http://www.iif.com/ (accessed 18 August 2018).

Londono, Juan, and Beth Anne Wilson. 2018. "Understanding Global Volatility." IFDP Notes. Washington, DC: Board of Governors of the Federal Reserve System.

Miniane, Jacques, and John Rogers. 2007. "Capital Controls and the International Transmission of US Monetary Shocks.” Journal of Money, Credit and Banking 39 (5): 1003-35.

Mundell, Robert. 1963. "Capital Mobility and Stabilization Policy under Fixed and Flexible Exchange Rates." Canadian Journal of Economics and Political Science 29 (4): 475-85.

Obstfeld, Maurice, Jonathan Ostry, and Mahvash Qureshi. 2017. "A Tie That Binds: Revisiting the Trilemma in Emerging Market Economies.” IMF Working Paper WP/17/130. Washington, DC: International Monetary Fund. 
Philippon, Thomas, Jeromin Zettelmeyer, and Eduardo Borensztein. 2001. "Monetary Independence in Emerging Markets: Does the Exchange Rate Regime Make a Difference?" IMF Working Paper WP/10/11. Washington, DC: International Monetary Fund.

The Political Risk Group. http://www.prsgroup.com/ (accessed 18 August 2018).

Pontines, Victor, and Ramkishen Rajan. 2011. "Foreign Exchange Market Intervention and Reserve Accumulation in Emerging Asia: Is There Evidence of Fear of Appreciation?" Economics Letters 111: 252-55.

Quinn, Dennis, and Maria Toyoda. 2008. "Does Capital Account Liberalization Lead to Economic Growth?” Review of Financial Studies 21 (3): 1403-49.

Reinhart, Carmen, and Kenneth Rogoff. 2004. "The Modern History of Exchange Rate Arrangements: A Reinterpretation.” Quarterly Journal of Economics 199: 1-48.

Rey, Helene. 2015. "Dilemma Not Trilemma: The Global Financial Cycle and Monetary Policy Independence." NBER Working Paper No. 21162. Cambridge, MA: National Bureau of Economic Research.

2016. "International Channels of Transmission of Monetary Policy and the Mundellian Trilemma." IMF Economic Review 6: 6-35.

Ricci, Lucca, and Wei Shi. 2016. "Trilemma or Dilemma: Inspecting the Heterogeneous Response of Local Currency Interest Rates to Foreign Rates." IMF Working Paper WP/16/75. Washington, DC: International Monetary Fund.

Shambaugh, Jay. 2004. "The Effect of Fixed Exchange Rates on Monetary Policy." Quarterly Journal of Economics 119 (1): 301-52.

Shin, Hyun. 2016. "The Bank/Capital Markets Nexus Goes Global.” Speech made at the London School of Economics and Political Science. 15 November. http://www.bis.org/speeches/sp161115.pdf.

Tovar, Camilo. 2004. "Devaluations, Output and the Balance Sheet Effect: A Structural Econometric Analysis." Unpublished.

Wu, Jing Cynthia, and Fan Dora Xia. 2016. "Measuring the Macroeconomic Impact of Monetary Policy at the Zero Lower Bound." Journal of Money, Credit and Banking 48 (2-3): 253-91.

Yasuba, Yasukichi. 1988. "The Japanese Economic and Economic Policy in the 1930s." In Recovery from the Great Depression, edited by Noel Butlin and Robert Gregory, 136-51. Cambridge: Cambridge University Press. 


\section{Exchange Rates and Insulation in Emerging Markets}

Whether or not flexible exchange rates can protect emerging markets from external shocks has long been a highly contentious issue in international economics. A number of recent theoretical and empirical studies cast doubt on the shock-mitigating benefits of exchange rate flexibility. On the other hand, a 2017 paper by Maurice Obstfeld, Jonathan Ostry, and Mahvash Qureshi finds that flexible exchange rates promote output and financial stability in the face of global shocks. The authors of this paper join the debate by significantly extending and performing sensitivity checks on the 2017 paper. Overall, their findings confirm that flexible exchange rate regimes are better at insulating emerging markets from external shocks.

\section{About the Asian Development Bank}

ADB is committed to achieving a prosperous, inclusive, resilient, and sustainable Asia and the Pacific, while sustaining its efforts to eradicate extreme poverty. Established in 1966, it is owned by 68 members -49 from the region. Its main instruments for helping its developing member countries are policy dialogue, loans, equity investments, guarantees, grants, and technical assistance. 